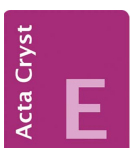

CRYSTALLOGRAPHIC COMMUNICATIONS

ISSN 2056-9890

Received 16 September 2016

Accepted 29 September 2016

Edited by M. Zeller, Purdue University, USA

Keywords: crystal structure; amidinate ligands; guanidinate ligands; amidinoguanidinate; lithium; holmium.

CCDC references: $1414136 ; 1414137$

Supporting information: this article has supporting information at journals.iucr.org/e

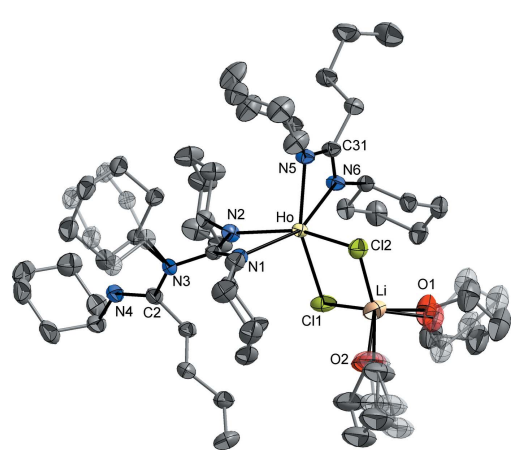

OPEN $\odot$ ACCESS

\section{Formation and structure of the first metal complexes comprising amidinoguanidinate ligands}

\author{
Farid M. Sroor, ${ }^{a}$ Phil Liebing, ${ }^{b}$ Cristian G. Hrib, ${ }^{b}$ Daniel Gräsing, ${ }^{b}$ Liane Hilfert ${ }^{b}$ \\ and Frank T. Edelmann ${ }^{\mathbf{b}}$ * \\ aOrganometallic and Organometalloid Chemistry Department, National Research, Centre, 12622 Dokki, Cairo, Egypt,
and ${ }^{\mathbf{b}}$ Chemisches Institut der Otto-von-Guericke-Universität Magdeburg, Universitätsplatz 2, 39106 Magdeburg,
Germany. ${ }^{*}$ Correspondence e-mail: frank.edelmann@ovgu.de
}

The first metal complexes comprising amidinoguanidinate ligands have been prepared and structurally characterized, namely $\operatorname{bis}\left[\mu-N, N^{\prime}, N^{\prime \prime}, N^{\prime \prime \prime}\right.$-tetraisopropyl-1-(1-butylamidinato)guanidinato- $\left.\kappa^{3} N^{1}, N^{2}: N^{2}\right]$ bis[(tetrahydrofuran)lithium], [ $\mathrm{Li}_{2}\left(\mathrm{C}_{18} \mathrm{H}_{37} \mathrm{~N}_{4}\right)_{2}\left(\mathrm{C}_{4} \mathrm{H}_{8} \mathrm{O}\right)_{2}$ ], (2), and [bis(tetrahydrofuran)lithium]di- $\mu$-chlorido- $\left\{\left(N, N^{\prime}\right.\right.$-dicyclohexyl-1-butylamidinato- $\left.\kappa^{2} N^{1}, N^{2}\right)\left[N, N^{\prime}, N^{\prime \prime}, N^{\prime \prime \prime}\right.$-tetracyclohexyl-1-(1-butylamidinato)guanidinato- $\left.\kappa^{2} N^{1}, N^{2}\right]$ holmate(III)\}, [HoLiCl $2^{-}$ $\left.\left(\mathrm{C}_{4} \mathrm{H}_{8} \mathrm{O}\right)_{2}\left(\mathrm{C}_{17} \mathrm{H}_{31} \mathrm{~N}_{2}\right)\left(\mathrm{C}_{30} \mathrm{H}_{53} \mathrm{~N}_{4}\right)\right]$, (3). The novel lithium amidinoguanidinate precursors $\mathrm{Li}\left[{ }^{n} \mathrm{BuC}(=\mathrm{N} R)(\mathrm{N} R) \mathrm{C}(\mathrm{N} R)_{2}\right]\left[\mathbf{1}: R=\mathrm{Cy}\left(\right.\right.$ cyclohexyl), 2: $\left.R={ }^{i} \mathrm{Pr}\right)$ were obtained by treatment of $N, N^{\prime}$-diorganocarbodiimides, $R-\mathrm{N}=\mathrm{C}=\mathrm{N}-R$ $\left(R={ }^{i} \operatorname{Pr}, \mathrm{Cy}\right)$, with 0.5 equivalents of $n$-butyllithium under well-defined reaction conditions. An X-ray diffraction study of 2 revealed a ladder-type dimeric structure in the solid state. Reaction of anhydrous holmium(III) chloride with in situ-prepared $\mathbf{2}$ afforded the unexpected holmium 'ate' complex $\left[{ }^{n} \mathrm{BuC}(=\mathrm{NCy})(\mathrm{NCy}) \mathrm{C}(\mathrm{NCy})_{2}\right] \mathrm{Ho}\left[{ }^{n} \mathrm{BuC}(\mathrm{NCy})_{2}\right](\mu-\mathrm{Cl})_{2} \mathrm{Li}(\mathrm{THF})_{2}$ (3) in $71 \%$ yield. An X-ray crystal structure determination of $\mathbf{3}$ showed that this complex contains both an amidinate ligand and the new amidinoguanidinate ligand.

\section{Chemical context}

Anionic $N$-chelating donor ligands such as the amidinates $\left[R \mathrm{C}(\mathrm{N} R)_{2}\right]^{-}$and the guanidinates $\left[R_{2} \mathrm{NC}(\mathrm{N} R)_{2}\right]^{-}$have gained tremendous importance in various fields of organometallic and coordination chemistry during the past two decades. Both types of $\mathrm{N}$-chelating ligands are often regarded as 'steric cyclopentadienyl equivalents' (Bailey \& Pace, 2001; Collins, 2011; Edelmann, 2013). Meanwhile, amidinato and guanidinato complexes are known for virtually every metallic element in the Periodic Table ranging from lithium to uranium (Edelmann, 2009, 2012, 2013; Trifonov, 2010). Amidinate and guanidinate ligands have been successfully employed in the stabilization of unusual oxidation states such as magnesium(I) and iron(I) as well as the design of various homogeneous catalysts (Collins, 2011; Edelmann, 2013). Alkyl-substituted amidinate and guanidinate complexes of various metals have also been established as ALD and MOCVD precursors for the deposition of thin layers of metals, metal oxides, metal nitrides etc. (Devi, 2013). Formally, the amidinate anion is the nitrogen analogue of the carboxylate anion, while guanidinates are similarly related to the carbamates. However, in contrast to the carboxylates and carbamates, the steric properties of amidinates and guanidinates can be widely tuned through the use of different substituents, both at the outer nitrogen atoms as well as at the central carbon atom of the NCN unit. Lithium 


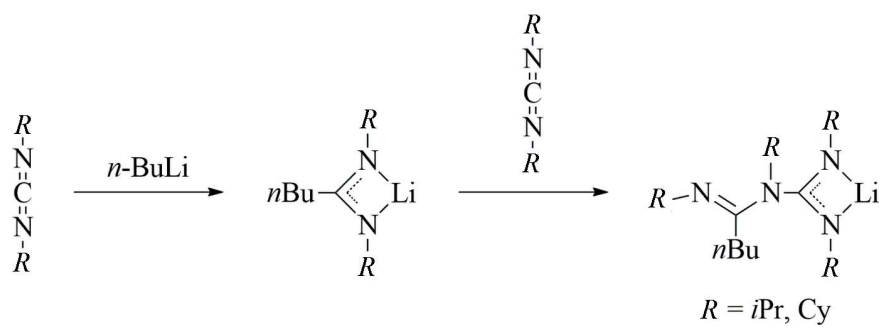

Figure 1

Reaction scheme: Formation of amidinoguanidinate ligands from the reaction of $n$-butyllithium with excess carbodiimide.

amidinates are normally prepared in a straightforward manner by addition of lithium alkyls to $N, N^{\prime}$-diorganocarbodiimides in a 1:1 molar ratio, while lithium guanidinates are formed when lithium- $N, N$-dialkylamides are added to $N, N^{\prime}$-diorganocarbodiimides (Stalke et al., 1992; Aharonovich et al., 2008; Chlupatý et al., 2011; Nevoralová et al., 2013; Hong et al., 2013). All these reactions are generally quite straightforward and afford the desired products in high yields. We have now discovered that, under certain conditions, reactions of lithium alkyls with $N, N^{\prime}$ diorganocarbodiimides can afford different products which can be named 'amidinoguanidinates' ( $c f$. reaction scheme, Fig. 1). These can even become the major reaction products when the stoichiometry of the reactands is changed from 1:1 to $1: 2$, i.e. when the $N, N^{\prime}$-diorganocarbodiimide is used in a twofold molar excess. We report here the synthesis and characterization of the first metal complexes comprising 'amidinoguanidinate' ligands which can be viewed as dimers of the amidinate anions. The first amidinoguanidinate complexes described here include the lithium precursors $\mathrm{Li}\left[{ }^{n} \mathrm{BuC}(=\mathrm{N} R)(\mathrm{N} R) \mathrm{C}(\mathrm{N} R)_{2}\right] \quad(\mathbf{1}: R=\mathrm{Cy}$ (cyclohexyl), 2: $\left.R={ }^{i} \mathrm{Pr}\right)$ and the holmium(III) 'ate' complex $\left[{ }^{n} \mathrm{Bu}-\mathrm{C}(=\mathrm{NCy})-\right.$ $\left.(\mathrm{NCy}) \mathrm{C}(\mathrm{NCy})_{2}\right] \mathrm{Ho}\left[{ }^{n} \mathrm{BuC}(\mathrm{NCy})_{2}\right](\mu-\mathrm{Cl})_{2} \mathrm{Li}(\mathrm{THF})_{2}(3)$.

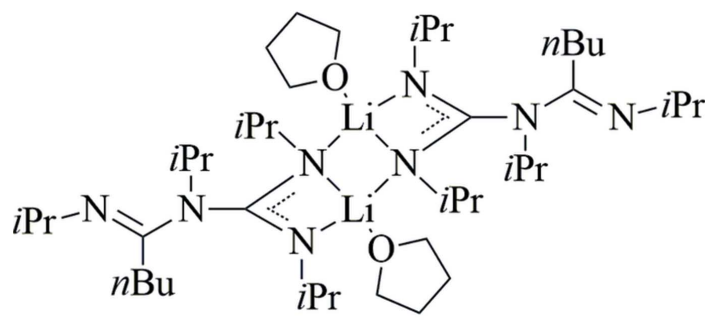

2

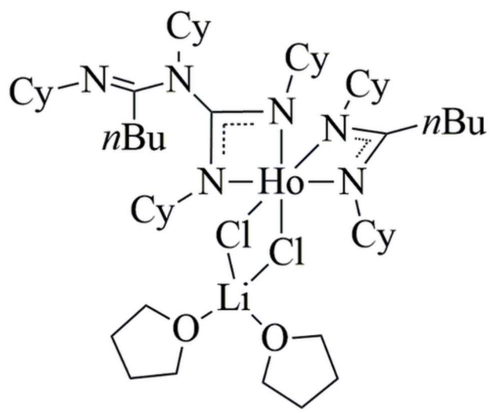

3
A reaction between $N, N^{\prime}$-dicyclohexylcarbodiimide and ${ }^{n} \mathrm{BuLi}$ in a 2:1 molar ratio in THF afforded the first lithium amidinoguanidinate, $\quad \mathrm{Li}\left[{ }^{n} \mathrm{BuC}(=\mathrm{NCy})(\mathrm{NCy}) \mathrm{C}(\mathrm{NCy})_{2}\right] \cdot \mathrm{THF}$ (1), in $60 \%$ yield. This reaction represents the first case of dimerization of a carbodiimide under formation of a novel amidinoguanidinate anion. The lithium-amidinoguanidinate salt $\mathbf{1}$ is partially soluble in THF, $\mathrm{Et}_{2} \mathrm{O}$, and DME and slightly soluble even in toluene and $n$-pentane. The new sterically bulky amidinoguanidinate $\mathbf{1}$ has been fully characterized by spectroscopic methods and elemental analysis to confirm the product as shown in Fig. 1 . DMSO- $d_{6}$ (DMSO $=$ dimethyl sulfoxide) was found to be the best solvent for measuring the NMR spectra of $\mathrm{Li}\left[{ }^{n} \mathrm{BuC}(=\mathrm{NCy})(\mathrm{NCy}) \mathrm{C}(\mathrm{NCy})_{2}\right] \cdot \mathrm{THF}$. A mass spectrum of 1 showed only fragments for the monomeric compound. Interestingly, the reaction using $N, N^{\prime}$-dicyclohexylcarbodiimide and $n$-butyllithium in THF according to Fig. 1 represents the only case thus far where a pure amidinoguanidinate salt (1) could be isolated. A similar reaction carried out with $N, N^{\prime}$-diisopropylcarbodiimide produced the isopropyl-substituted amidinoguanidinate salt 2 in $>70 \%$ yield, although NMR data indicated the presence of significant amounts of an impurity, presumably the 'normal' lithium amidinate $\mathrm{Li}\left[{ }^{n} \mathrm{BuC}\left(\mathrm{N}^{i} \mathrm{Pr}\right)_{2}\right]$, which could not be separated by fractional crystallization from solvents like THF, DME or diethyl ether. However, occasionally a small amount of well-formed single-crystals of $\mathbf{2}$ were obtained directly from the reaction mixture which allowed a structural characterization of the new amidinoguanidinates through X-ray diffraction. Apparently the formation of the new amidinoguanidinate anions is critically influenced not only by the stoichiometric ratio of the starting materials, but also by the substituents at the $N$-atoms and the solvents employed. The solvent effect became apparent when reactions of $N, N^{\prime}$-dicyclohexylcarbodiimide with 0.5 or 0.3 equiv. of $n$-butyllithium were carried out in $\mathrm{Et}_{2} \mathrm{O}$ solution. Using this solvent, the reactions produced a variable mixture of amidinoguanidinate and amidinate salts, $\operatorname{Li}\left[{ }^{n} \mathrm{BuC}(=\mathrm{NCy})(\mathrm{NCy}) \mathrm{C}(\mathrm{NCy})_{2}\right]$ and $\mathrm{Li}\left[{ }^{n} \mathrm{BuC}(\mathrm{NCy})_{2}\right]$, respectively, as illustrated in the reaction scheme (Fig. 1). This was clearly indicated by the rather 'messy' NMR spectra of the reaction products. Attempts to separate the product mixture by fractional crystallization from THF, DME, or diethyl ether were unsuccessful.

The presence of both types of anions in the reaction mixture obtained was also confirmed by the subsequent reaction of the in situ-prepared mixture of $\mathrm{Li}\left[{ }^{n} \mathrm{BuC}(=\mathrm{NCy})(\mathrm{NCy}) \mathrm{C}(\mathrm{NCy})_{2}\right]$ and $\mathrm{Li}\left[{ }^{n} \mathrm{BuC}(\mathrm{NCy})_{2}\right]$ with anhydrous $\mathrm{HoCl}_{3}$. In detail, treatment of $N, N^{\prime}$-dicyclohexylcarbodiimide with 0.5 equiv. of ${ }^{n} \mathrm{BuLi}$ in $\mathrm{Et}_{2} \mathrm{O}$ followed by addition of anhydrous $\mathrm{HoCl}_{3}$ (Freeman \& Smith, 1958) in THF produced a yellow solution. Separation of the $\mathrm{LiCl}$ by-product and recrystallization from $n$-pentane afforded the unexpected holmium complex $\left[{ }^{n} \mathrm{BuC}(=\mathrm{NCy})(\mathrm{NCy}) \mathrm{C}(\mathrm{NCy})_{2}\right] \mathrm{Ho}\left[{ }^{n} \mathrm{BuC}(\mathrm{NCy})_{2}\right](\mu-\mathrm{Cl})_{2} \mathrm{Li}-$ $(\mathrm{THF})_{2}(\mathbf{3})$ in $71 \%$ yield. This compound is a mixed-ligand complex containing both the new amidinoguanidinato ligand and the normal amidinato ligand $\left[{ }^{n} \mathrm{BuC}(\mathrm{NCy})_{2}\right]^{-}$in the coordination sphere of holmium. Compound $\mathbf{3}$ was fully characterized by its IR spectrum, elemental analysis and 


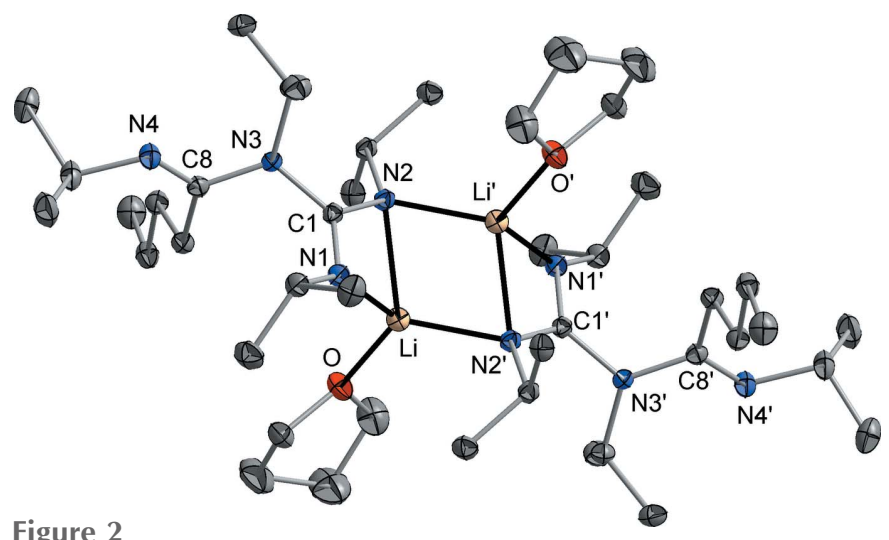

Figure

The molecular structure of compound 2 in the crystal. Displacement ellipsoids are drawn at the $50 \%$ probability level and $\mathrm{H}$ atoms have been omitted for clarity. Symmetry operator to generate equivalent atoms: $2-x, 1-y,-z$.

single-crystal X-ray diffraction. As a result of the highly paramagnetic nature of the $\mathrm{Ho}^{3+}$ ion, it was impossible to obtain interpretable NMR data for 3. Yellow, air- and moisture-sensitive, needle-like single-crystals of $\mathbf{3}$ were obtained by slowly cooling a saturated solution in $n$-pentane to $268 \mathrm{~K}$.

In summarizing the results reported here, we prepared the first metal complexes containing novel amidinoguanidinate ligands obtained by dimerization of $N, N^{\prime}$-diorganocarbodiimides in the presences of sub-stoichiometric amounts of $n$-butyllithium. The cyclohexyl-substituted lithium-amidinoguanidinate salt $\mathrm{Li}\left[{ }^{n} \mathrm{BuC}(=\mathrm{NCy})(\mathrm{NCy}) \mathrm{C}(\mathrm{NCy})_{2}\right] \cdot \mathrm{THF}(\mathbf{1})$ is readily available as a pure solid in fairly good yield $(60 \%)$. This compound could play an interesting role as a precursor for the synthesis of new transition metal and lanthanide amidinoguanidinate complexes. The first lanthanide complex comprising the new ligand system is the holmium 'ate' complex $\left[{ }^{n} \mathrm{BuC}(=\mathrm{NCy})(\mathrm{NCy}) \mathrm{C}(\mathrm{NCy})_{2}\right] \mathrm{Ho}\left[{ }^{n} \mathrm{BuC}(\mathrm{NCy})_{2}\right](\mu-$ $\mathrm{Cl})_{2} \operatorname{Li}(\mathrm{THF})_{2}(\mathbf{3})$.

\section{Structural commentary}

The crystal structure determination of $\mathbf{2}$ revealed the presence of ladder-type centrosymmetric dimers (space group $P 2_{1} / \mathrm{c}$, $Z=2$ ), which is the most characteristic structural motif of most previously characterized lithium amidinates and guanidinates (Stalke et al., 1992; Snaith \& Wright, 1995; Downard \& Chivers, 2001). Fig. 2 shows the molecular structure of compound 2, while crystallographic data are summarized in Table 1. The central building unit of the dimer is a typical planar $\mathrm{Li}_{2} \mathrm{~N}_{2}$ ring, formed by $\mu$-bridging coordination of one of the guanidinate $\mathrm{N}$ atoms $(\mathrm{N} 2)$. The $\mathrm{Li}-\mathrm{N}$ distances within this ring are 2.0528 (17) and 2.1559 (17) $\AA$ and therefore in the expected range. The second $\mathrm{N}$ atom of the guanidinate unit (N1) is attached to only one $\mathrm{Li}$ atom with a shorter $\mathrm{Li}-\mathrm{N}$ bond of 2.0177 (18) $\AA$. Through this $\mu-\kappa^{3} N, N^{\prime}: N$-coordination mode of the guanidinato moiety, a 'ladder' consisting of three four-membered rings is formed. By coordination of a solvent THF molecule, a typical distorted tetrahedral coordination of

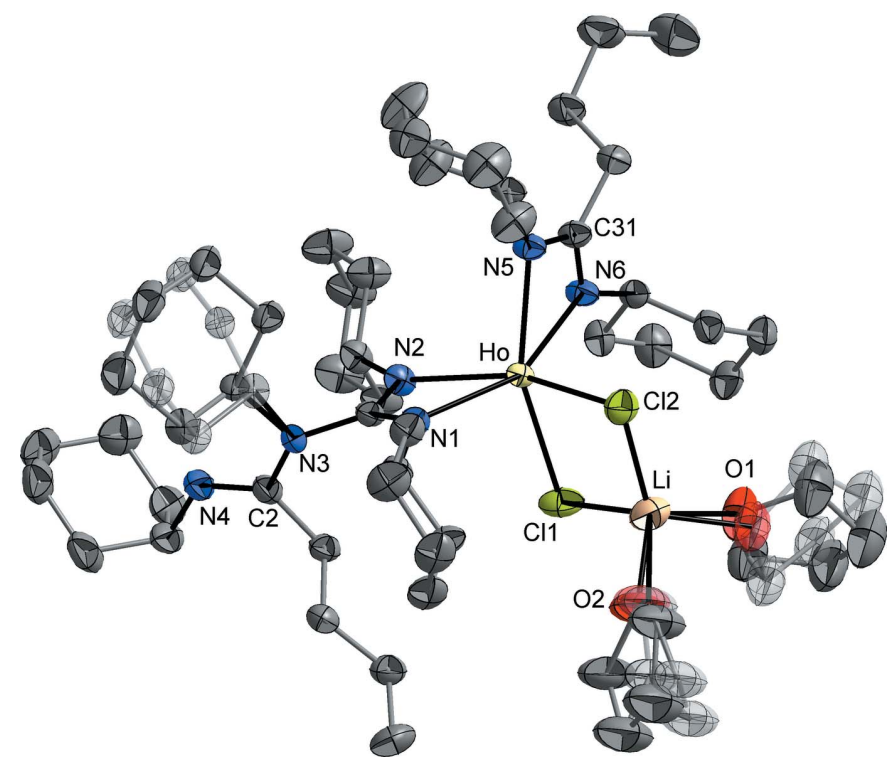

Figure 3

The molecular structure of compound $\mathbf{3}$ in the crystal, illustrating the disorder of one cyclohexyl group and both THF ligands. Displacement ellipsoids drawn at the $50 \%$ probability level and $\mathrm{H}$ atoms have been omitted for clarity.

the $\mathrm{Li}$ atom is completed. The free $\mathrm{N}$ donor of the amidinate unit (N4) does not contribute to coordinative saturation of the $\mathrm{Li}$ atom. The bonds $\mathrm{C} 1-\mathrm{N} 1$ [1.3197 (12) $\mathrm{A}]$ and $\mathrm{C} 1-\mathrm{N} 2$ [1.3396 (11) $\AA$ ] are similar in length, indicating a common delocalization of the negative charge within the Li-coordinating $\mathrm{N}-\mathrm{C}-\mathrm{N}$ fragment. By contrast, the third $\mathrm{C}-\mathrm{N}$ bond of the guanidinate unit $\mathrm{C} 1-\mathrm{N} 3$ is considerably longer at 1.4528 (11) $\AA$ and can therefore be interpreted as a pure single bond. The 1-butylamidinate fragment does not show any delocalization of the $\pi$-electron density, with one distinct double bond [C8-N4, 1.2808 (12) $\AA$ ] and one single bond [C8-N3, 1.3940 (11) A] . The amidinate C3-C8-N3 fragment is twisted out of the guanidinate $\mathrm{C} 1 / \mathrm{N} 1 / \mathrm{N} 2 / \mathrm{N} 3$ plane by approx. $75^{\circ}$, similar to that found earlier for this type of ligands (Zhou et al., 1998; Wood et al., 1999; Lu et al., 2001).

The holmium complex $\mathbf{3}$ crystallizes in the triclinic space group $P \overline{1}$ with one molecule in the asymmetric unit. The molecular structure is shown in Fig. 3. The X-ray diffraction study revealed the presence of an 'ate' complex formed through retention of a $\left[\mathrm{LiCl}(\mathrm{THF})_{2}\right]$ fragment by the five-coordinate unit $\left[{ }^{n} \mathrm{BuC}(=\mathrm{NCy})(\mathrm{NCy}) \mathrm{C}(\mathrm{NCy})_{2}\right] \mathrm{Ho}\left[{ }^{n} \mathrm{BuC}\right.$ $\left.(\mathrm{NCy})_{2}\right] \mathrm{Cl}$. The phenomenon of 'ate' complex formation via retention of alkali metal halides in the products is quite common in organolanthanide chemistry (Edelmann, 2006). It can be traced back to the strong tendency of the large $\mathrm{Ln}^{3+}$ ions to adopt high coordination numbers. In the resulting sixcoordinate bimetallic complex $\mathbf{3}$, the central holmium(III) ion is coordinated by two $\mu$-bridging chloride ions, one chelating amidinoguanidinate ligand and one chelating amidinate ligand. The Ho atom is located in the C1N1N2N3 plane of the amidinoguanidinate ligand and, just like in the case of the lithium derivative $\mathbf{2}$, the amidinate $\mathrm{N}$ atom $\mathrm{N} 4$ does not contribute to metal coordination. The $\mathrm{Ho}-\mathrm{N}$ distances are in 
a narrow range of 2.327 (3)-2.354 (3) $\AA$ that is in good agreement with the values observed in related lanthanide amidinate and guanidinate complexes (Edelmann, 2009, 2012). The same applies to the corresponding coordination angles $\mathrm{N} 1-\mathrm{Ho}-\mathrm{N} 2\left[57.0(1)^{\circ}\right]$ and $\mathrm{N} 5-\mathrm{Ho}-\mathrm{N} 6\left[57.3(1)^{\circ}\right]$. The guanidinate and the amidinate moiety in compound $\mathbf{3}$ are arranged nearly perpendicular to each other, gaining a minimal contact between the bulky cyclohexyl substituents. The $\left[\mathrm{LiCl}_{2}(\mathrm{THF})_{2}\right]$ fragment is attached to the Ho atom in a formally chelating mode, leading to the formation of a regular kite-shaped $\mathrm{Ho} / \mathrm{Cl} 1 / \mathrm{Li} / \mathrm{Cl} 2$ ring $[\mathrm{Ho}-\mathrm{Cl} 2.6326(13)$ and $2.6453(15) \AA$, $\mathrm{Ho}-\mathrm{Cl}-\mathrm{Li} 87.0(2)$ and $\left.88.0(3)^{\circ}\right]$. The $\mathrm{Li}$ atom exhibits a typical tetrahedral coordination by the two $\mu$-bridging $\mathrm{Cl}$ atoms and two THF ligands. Within the chelating NCN units of the amidinato and the amidinoguanidinato ligands, the $\mathrm{C}-\mathrm{N}$ distances are nearly equal [1.324 (5)-1.336 (5) $\AA$ ], indicating a typical $\pi$-electron delocalization within these units. The conformation of the amidinatoguanidinate ligand is very similar to that in compound 2 (angle between guanidinate and amidinate plane approx. $75^{\circ}$ ), and the localization of single and double bonds within the 1-butylamidinate backbone is identical with that in the lithium derivative [C-N $1.272(5)-1.429$ (5) $\mathrm{A}]$.

\section{Supramolecular features}

Due to an effective 'packaging' of the molecules by the sterically demanding alkyl substituents, both title compounds do not feature any specific intermolecular interactions. In the lithium derivative $\mathbf{2}$, the closest intermolecular contacts are between two isopropyl- $\mathrm{CH}_{3}$ groups [C3 …C10, 3.740 (3) $\mathrm{A}$ ] and between an isopropyl- $\mathrm{CH}_{3}$ and a butyl- $\mathrm{CH}_{3}$ group $[\mathrm{C} 13 \cdots \mathrm{C} 18,3.744$ (4) $\AA$ ] . The crystal structure of the holmium complex 3 comprises a close package of cyclohexyl groups, butyl groups and THF ligands with a minimal $\mathrm{H}_{2} \mathrm{C} \cdots \mathrm{CH}_{2}$ distance of 3.64 (4) $\AA$, and one $\mathrm{H}_{2} \mathrm{C} \cdots \mathrm{CH}_{3}$ contact of at least 3.73 (6) $\AA$ ( 66 and $\mathrm{C} 21 B$ of disordered cyclohexyl group).

\section{Database survey}

For other structurally characterized lithium amidinates and guanidinates, see: Stalke et al. (1992), Aharonovich et al. (2008), Chlupatý et al. (2011), Nevoralová et al. (2013) and Hong et al. (2013).

For other lanthanide(III) complexes with amidinate ligands, see: Richter et al. (2004), Edelmann $(2009,2012)$ and Deacon et al. (2014).

\section{Synthesis and crystallization}

General Procedures: All reactions were carried out under an inert atmosphere of dry argon employing standard Schlenk and glovebox techniques. THF and $n$-pentane were distilled from sodium/benzophenone under nitrogen atmosphere prior to use. All glassware was oven-dried at $393 \mathrm{~K}$ for at least $24 \mathrm{~h}$, assembled while hot, and cooled under high vacuum prior to use. Anhydrous holmium(III) chloride was prepared according to the literature method (Freeman \& Smith, 1958). $n$-Butyllithium solution, $N, N^{\prime}$-diisopropylcarbodiimide and $N, N^{\prime}$-dicyclohexylcarbodiimide were purchased from Aldrich and used as received. ${ }^{1} \mathrm{H}$ NMR $(400 \mathrm{MHz})$ and ${ }^{13} \mathrm{C}$ NMR $(100.6 \mathrm{MHz})$ spectra were recorded in DMSO- $d_{6}$ solution on a Bruker DPX 400 spectrometer at $298 \mathrm{~K}$. Chemical shifts are referenced to TMS. IR spectra were recorded using $\mathrm{KBr}$ pellets on a Perkin Elmer FT-IR spectrometer system 2000 between $4000 \mathrm{~cm}^{-1}$ and $400 \mathrm{~cm}^{-1}$. Microanalyses (C, $\mathrm{H}$ and $\mathrm{N}$ ) of compounds $\mathbf{1}$ and $\mathbf{3}$ were performed using a Leco CHNS 932 apparatus.

Synthesis of $\operatorname{Li}\left[{ }^{n} \mathrm{BuC}(=\mathrm{NCy})(\mathrm{NCy}) \mathrm{C}(\mathrm{NCy})_{2}\right] \cdot \mathrm{THF}(1)$ : A solution of $N, N^{\prime}$-dicyclohexylcarbodiimide $(10.30 \mathrm{~g}, 50 \mathrm{mmol})$ in $100 \mathrm{ml}$ of THF at $253 \mathrm{~K}$ was treated slowly with $n$-butyllithium ( $16 \mathrm{ml}, 1.6 \mathrm{M}$ solution in hexanes). The reaction mixture was stirred for $10 \mathrm{~min}$ at $253 \mathrm{~K}$, then warmed to room temperature and stirred overnight to give a white suspension in THF. The solvent was removed under vacuum affording $\mathbf{1}$ as white solid. Yield: $16.4 \mathrm{~g}, 60 \%$. Elemental analysis for $\mathrm{C}_{34} \mathrm{H}_{61} \mathrm{LiN}_{4} \mathrm{O}\left(548.83 \mathrm{~g} \mathrm{~mol}^{-1}\right)$ : C, 74.41; H, 11.20; N, 10.21; found $\mathrm{C}, 74.82 ; \mathrm{H}, 10.85 ; \mathrm{N}, 10.50 .{ }^{1} \mathrm{H}$ NMR $(400 \mathrm{MHz}$, $\left.\left(\mathrm{CD}_{3}\right)_{2} \mathrm{SO}, 298 \mathrm{~K}\right): \delta$ (p.p.m.) $3.84(m, 1 \mathrm{H}, \mathrm{CH}, \mathrm{Cy}), 3.60(m$, 4H, THF), 3.43 ( $m, 1 \mathrm{H}, \mathrm{CH}, \mathrm{Cy}), 3.04-3.18$ ( $m, 2 \mathrm{H}, \mathrm{CH}, \mathrm{Cy})$, $2.66\left(m, 1 \mathrm{H}, \mathrm{CH}_{2},{ }^{n} \mathrm{Bu}\right), 2.33\left(m, 1 \mathrm{H}, \mathrm{CH}_{2},{ }^{n} \mathrm{Bu}\right), 2.09(m, 2 \mathrm{H}$, $\left.\mathrm{CH}_{2},{ }^{n} \mathrm{Bu}\right), 1.84\left(m, 2 \mathrm{H}, \mathrm{CH}_{2},{ }^{n} \mathrm{Bu}\right), 1.76(m, 4 \mathrm{H}, \mathrm{THF}), 1.65$ $\left(m, 8 \mathrm{H}, \mathrm{CH}_{2}, \mathrm{Cy}\right), 1.52\left(m, 6 \mathrm{H}, \mathrm{CH}_{2}, \mathrm{Cy}\right), 1.26\left(m, 26 \mathrm{H}, \mathrm{CH}_{2}\right.$, $\mathrm{Cy}), 0.85\left(m, 3 \mathrm{H}, \mathrm{CH}_{3},{ }^{n} \mathrm{Bu}\right) ;{ }^{13} \mathrm{C}$ NMR $\left(100.6 \mathrm{MHz}, \mathrm{C}_{6} \mathrm{D}_{6}\right.$, 298 K): $\delta$ (p.p.m.) 155.3 (NCN), $145.1(\mathrm{NCN}), 67.0$ (THF), 55.4 $(\mathrm{CH}, \mathrm{Cy}), 54.2(\mathrm{CH}, \mathrm{Cy}), 49.3(\mathrm{CH}, \mathrm{Cy}), 35.7\left(\mathrm{CH}_{2}, \mathrm{Cy}\right), 35.1$ $\left(\mathrm{CH}_{2}, \mathrm{Cy}\right), 34.8\left(\mathrm{CH}_{2}, \mathrm{Cy}\right), 34.5\left(\mathrm{CH}_{2},{ }^{n} \mathrm{Bu}\right), 30.7\left(\mathrm{CH}_{2},{ }^{n} \mathrm{Bu}\right)$, $29.5\left(\mathrm{CH}_{2},{ }^{n} \mathrm{Bu}\right), 25.8$ (THF), $24.9\left(\mathrm{CH}_{2}, \mathrm{Cy}\right), 22.6\left(\mathrm{CH}_{2}, \mathrm{Cy}\right)$, $22.1\left(\mathrm{CH}_{2}, \mathrm{Cy}\right), 13.8\left(\mathrm{CH}_{3}\right)$. MS (EI, $\left.M=548.50\right)$ : $m / z(\%) 125.2(27)\left[\mathrm{Cy}+\mathrm{C}_{3} \mathrm{H}_{6}\right]^{+}, 153.2(88)[2 \mathrm{Cy}-\mathrm{Me}]^{2+}$, $183.3(20)[2 \mathrm{Cy}+\mathrm{Me}]^{2+}, 207.3(12)\left[\mathrm{C}(\mathrm{NCy})_{2}\right]^{+}, 222.3(62)$ $\left[\mathrm{C}(\mathrm{NCy})_{2}+\mathrm{Me}\right]^{2+}, 235.4(100)\left[\mathrm{C}(\mathrm{NCy})_{2}+\mathrm{C}_{2} \mathrm{H}_{5}\right]^{+}, 264.4(55)$ $\left[{ }^{n} \mathrm{Bu}+\mathrm{C}(\mathrm{NCy})_{2}\right]^{+}$. IR $(\mathrm{KBr}): n\left(\mathrm{~cm}^{-1}\right) 3449(w), 3327(w)$, $3225(w), 2927(v s), 2853(s), 2666(w), 2533(w), 2354(w), 2120$ $(w), 1959(w), 1645(m), 1578(w), 1516(m), 14450(m), 1367$ $(w), 1339(m), 1155(w), 1128(m), 1105(w), 1053(w), 1029$ $(w), 988(w), 919(w), 889(w), 845(w), 804(w), 748(w), 695$ $(w), 657(w), 640(w), 555(w), 502(w), 454(w)$.

Synthesis of $\operatorname{Li}\left[{ }^{n} \mathrm{BuC}\left(=\mathrm{N}^{i} \mathbf{P r}\right)\left(\mathrm{N}^{i} \mathbf{P r}\right) \mathbf{C}\left(\mathrm{N}^{i} \mathbf{P r}\right)_{2}\right]$.THF (2): In a similar manner as for compound $\mathbf{1}, N, N^{\prime}$-diisopropylcarbodiimide $(4.2 \mathrm{~g}, 50 \mathrm{mmol})$ was treated with $n$-butyllithium (10 ml, $2.5 M$ solution in hexanes) in THF solution $(80 \mathrm{ml})$. From this reaction $14.3 \mathrm{~g}$ of colorless 2 were isolated. X-ray quality single crystals (colorless rods) were occasionally obtained directly upon cooling of the reaction mixture to 278 K. However, NMR data showed that the bulk product was heavily contaminated with the lithium amidinate salt $\mathrm{Li}\left[{ }^{n} \mathrm{BuC}\left(\mathrm{N}^{i} \mathrm{Pr}\right)_{2}\right](10-20 \%)$ which could not be separated by fractional crystallization.

Synthesis of $\left[{ }^{n} \mathrm{BuC}(=\mathrm{NCy})(\mathrm{NCy}) \mathrm{C}(\mathrm{NCy})_{2}\right] \mathrm{Ho}\left[{ }^{n} \mathrm{BuC}-\right.$ $\left.(\mathbf{N C y})_{2}\right](\mu-\mathbf{C l})_{2} \mathbf{L i}(\mathbf{T H F})_{2}$ (3): A solution of anhydrous $\mathrm{HoCl}_{3}$ $(1.0 \mathrm{~g}, 3.6 \mathrm{mmol})$ in $50 \mathrm{ml}$ THF was added to a stirred $\mathrm{Et}_{2} \mathrm{O}$ solution $(80 \mathrm{ml})$ of an in situ-prepared mixture of $\mathrm{Li}\left[{ }^{n} \mathrm{Bu}\right.$ $\left.\mathrm{C}(=\mathrm{NCy})(\mathrm{NCy}) \mathrm{C}(\mathrm{NCy})_{2}\right] \quad$ and $\mathrm{Li}\left[{ }^{n} \mathrm{BuC}(\mathrm{NCy})_{2}\right] \quad\left(N, N^{\prime}\right.$-di- 
Table 1

Experimental details.

2

Crystal data

Chemical formula

$M_{\mathrm{r}}$

Crystal system, space group

Temperature $(\mathrm{K})$

$a, b, c(\AA)$

$\alpha, \beta, \gamma\left(^{\circ}\right)$

$V\left(\AA^{3}\right)$

Z

Radiation type

$\mu\left(\mathrm{mm}^{-1}\right)$

Crystal size (mm)

Data collection

Diffractometer

Absorption correction

$T_{\min }, T_{\max }$

No. of measured, independent and

observed $[I>2 \sigma(I)]$ reflections

$R_{\text {int }}$

$(\sin \theta / \lambda)_{\max }\left(\AA^{-1}\right)$

Refinement

$R\left[F^{2}>2 \sigma\left(F^{2}\right)\right], w R\left(F^{2}\right), S$

No. of reflections

No. of parameters

No. of restraints

$\mathrm{H}$-atom treatment

$\Delta \rho_{\max }, \Delta \rho_{\min }\left(\mathrm{e} \AA^{-3}\right)$

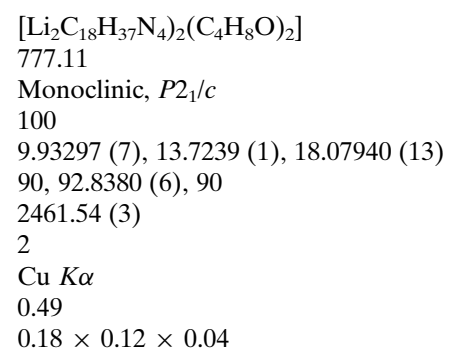

Agilent Xcalibur, Atlas, Nova

Multi-scan (CrysAlis PRO; Agilent, 2011)

$0.891,1.000$

30246, 5126, 4657

0.027

0.629

$0.036,0.096,1.05$

5126

263

0

$\mathrm{H}$-atom parameters constrained

$0.31,-0.21$
3

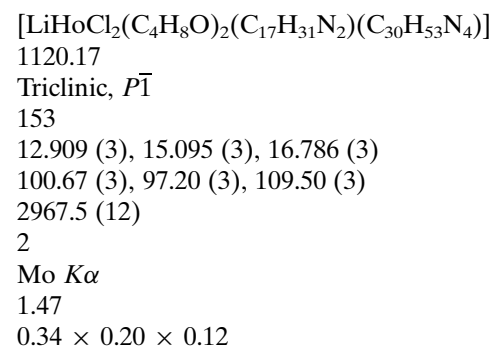

Stoe IPDS 2T

For a sphere $(X-A R E A$ and $X-R E D$; Stoe \& Cie, 2002)

$0.814,0.889$

29449, 12948, 9625

0.078

0.639

$0.048,0.074,0.91$

12948

751

552

$\mathrm{H}$-atom parameters constrained $0.98,-1.68$

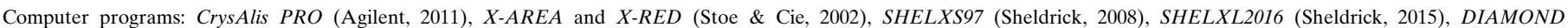
(Brandenburg, 1999) and publCIF (Westrip, 2010).

cyclohexylcarbodiimide $(10.30 \mathrm{~g}, 50 \mathrm{mmol})$ in $80 \mathrm{ml}$ of $\mathrm{Et}_{2} \mathrm{O}$ and was treated slowly with $n$-butyllithium $(16 \mathrm{~mL}, 1.6 \mathrm{M}$ solution in hexanes) at $253 \mathrm{~K}$. The reaction mixture was stirred for $3 \mathrm{~h}$ at room temperature. The solvents were evaporated under vacuum, and the residue was extracted with $20 \mathrm{ml} n$ pentane. Concentration and cooling of the filtered solution to $278 \mathrm{~K}$ afforded $\mathbf{3}$ as yellow, air- and moisture-sensitive, needlelike crystals. Yield: $2.8 \mathrm{~g}, 71 \%$. Elemental analysis for $\mathrm{C}_{55} \mathrm{H}_{100} \mathrm{Cl}_{2} \mathrm{HoLiN}_{6} \mathrm{O}_{2}\left(1120.22 \mathrm{~g} \mathrm{~mol}^{-1}\right): \mathrm{C}, 58.97 ; \mathrm{H}, 9.00 ; \mathrm{N}$, 7.50; found C, 58.92; H, 8.98; N, 7.44\%. IR (KBr): $n\left(\mathrm{~cm}^{-1}\right)$ $3321(w), 3223(w), 2929(v s), 2857(s), 2661(w), 2525(w), 2356$ $(w), 2118(w), 1952(w), 1577(w), 1518(m), 1367(w), 1156(w)$, $1129(m), 1108(w), 1085(w), 1055(w), 1045(w), 983(w), 922$ $(w), 892(w), 865(w), 820(w), 715(w), 657(w), 643(w), 553$ $(w), 505(w), 456(w)$. Meaningful NMR spectra could not be obtained due to the strong paramagnetism of the $\mathrm{Ho}^{3+}$ ion.

\section{Refinement}

Crystal data, data collection and structure refinement details are summarized in Table 1 . All $\mathrm{H}$ atoms were fixed geometrically and refined using a riding model with $U_{\text {iso }}(\mathrm{H})=1.2$ $U_{\text {eq }}(\mathrm{C})$. C- $\mathrm{H}$ distances in $\mathrm{CH}_{3}$ groups were constrained to $0.98 \AA$, those in $\mathrm{CH}_{2}$ groups to $0.99 \AA$ and those in $\mathrm{CH}$ groups to $1.00 \AA$. Methyl $\mathrm{H}$ atoms were allowed to rotate around the $\mathrm{C}-\mathrm{C}$ vector but not to tip to best fit the experimental electron density (AFIX 137 in SHELXL). In the crystallographic dataset of compound $\mathbf{3}$, the intensities of reflections (111) and (111) strongly disagreed with the structural model and were therefore omitted from the refinement. One of the cyclohexyl groups (C19-C24) and both THF ligands (O1, C48-C51 and $\mathrm{O} 2, \mathrm{C} 52-\mathrm{C} 55)$ in compound $\mathbf{3}$ are disordered. The aforementioned atoms were each split over two sites (site occupancy factors refined freely). Equivalent disordered THF and cyclohexyl moieties were restrained to have similar geometries (SAME restraint in SHELXLL), and $U_{\mathrm{ij}}$ components of ADPs were restrained to be similar for atoms closer than $1.7 \AA$ (SIMU restraint in SHELXL; the esd applied was $0.01 \AA^{2}$ ). Occupancy ratios refined to $0.760(6)$ and $0.240(6)$ for the cyclohexyl group (C19-C24), and to 0.663 (11) and 0.337 (11) (O1, C48-C51) and to 0.823 (11) and 0.177 (11) (O2, C52-C55) for the THF moieties.

\section{Acknowledgements}

Financial support of this work by the Otto-von-GuerickeUniversität Magdeburg is gratefully acknowledged. FMS is grateful to the Ministry of Higher Educational Scientific Research (MHESR), Egypt, and the German Academic Exchange Service (DAAD), Germany, for a PhD scholarship within the German Egyptian Research Long-Term Scholarship (GERLS) program. 


\section{References}

Agilent (2011). CrysAlis PRO. Agilent Technologies Ltd, Yarnton, England.

Aharonovich, S., Kapon, M., Botoshanski, M. \& Eisen, M. S. (2008). Organometallics, 27, 1869-1877.

Bailey, P. J. \& Pace, S. (2001). Coord. Chem. Rev. 214, 91-141.

Brandenburg, K. (1999). DIAMOND. Crystal Impact GbR, Bonn, Germany.

Chlupatý, T., Padělková, A., Lyčka, A. \& Růžička, A. (2011). J. Organomet. Chem. 696, 2346-2354.

Collins, S. (2011). Coord. Chem. Rev. 255, 118-138.

Deacon, G. B., Junk, P. C., Wang, J. \& Werner, D. (2014). Inorg. Chem. 53, 12553-12563.

Devi, A. (2013). Coord. Chem. Rev. 257, 3332-3384.

Downard, A. \& Chivers, T. (2001). Eur. J. Inorg. Chem. pp. 21932201.

Edelmann, F. T. (2006). In Comprehensive Organometallic Chemistry III, Vol. 3, Complexes of Scandium, Yttrium and the Lanthanide Elements, edited by R. H. Crabtree \& D. M. P. Mingos. Oxford: Elsevier.

Edelmann, F. T. (2009). Chem. Soc. Rev. 38, 2253-2268.

Edelmann, F. T. (2012). Chem. Soc. Rev. 41, 7657-7672.

Edelmann, F. T. (2013). Adv. Organomet. Chem. 61, 55-374.
Freeman, J. H. \& Smith, M. L. (1958). J. Inorg. Nucl. Chem. 7, 224 227.

Hong, J., Zhang, L., Wang, K., Chen, Z., Wu, L. \& Zhou, X. (2013). Organometallics, 32, 7312-7322.

Lu, Z. P., Yap, G. P. A. \& Richeson, D. S. (2001). Organometallics, 20, 706-712.

Nevoralová, J., Chlupatý, T., Padělková, A. \& Růžička, A. (2013). J. Organomet. Chem. 745-746, 186-189.

Richter, J., Feiling, J., Schmidt, H.-G., Noltemeyer, M., Brüser, W. \& Edelmann, F. T. (2004). Z. Anorg. Allg. Chem. 630, 1269-1275.

Sheldrick, G. M. (2008). Acta Cryst. A64, 112-122.

Sheldrick, G. M. (2015). Acta Cryst. C71, 3-8.

Snaith, R. \& Wright, D. S. (1995). In Lithium Chemistry, A Theoretical and Experimental Overview, edited by A. Sapse \& P. von R. Schleyer. New York: Wiley.

Stalke, D., Wedler, M. \& Edelmann, F. T. (1992). J. Organomet. Chem. 431, C1-C5.

Stoe \& Cie (2002). $X$-AREA and $X$-RED. Stoe \& Cie, Darmstadt, Germany.

Trifonov, A. A. (2010). Coord. Chem. Rev. 254, 1327-1347.

Westrip, S. P. (2010). J. Appl. Cryst. 43, 920-925.

Wood, D., Yap, G. P. A. \& Richeson, D. S. (1999). Inorg. Chem. 38, 5788-5794.

Zhou, Y., Yap, G. P. A. \& Richeson, D. S. (1998). Organometallics, 17, 4387-4391. 


\section{supporting information}

Acta Cryst. (2016). E72, 1526-1531 [https://doi.org/10.1107/S2056989016015322]

Formation and structure of the first metal complexes comprising amidinoguanidinate ligands

Farid M. Sroor, Phil Liebing, Cristian G. Hrib, Daniel Gräsing, Liane Hilfert and Frank T.

\section{Edelmann}

Computing details

Data collection: CrysAlis PRO (Agilent, 2011) for compound_2; X-AREA (Stoe \& Cie, 2002) for compound_3. Cell refinement: CrysAlis PRO (Agilent, 2011) for compound_2; X-AREA (Stoe \& Cie, 2002) for compound_3. Data reduction: CrysAlis PRO (Agilent, 2011) for compound_2; X-AREA and X-RED (Stoe \& Cie, 2002) for compound_3. For both compounds, program(s) used to solve structure: SHELXS97 (Sheldrick, 2008); program(s) used to refine structure: SHELXL2016 (Sheldrick, 2015). Molecular graphics: Diamond (Brandenburg, 1999) for compound_2; DIAMOND (Brandenburg, 1999) for compound_3. For both compounds, software used to prepare material for publication: publCIF (Westrip, 2010).

(compound_2) Bis[ $\mu \mu-N, N^{\prime}, N^{\prime \prime}, N^{\prime \prime \prime}$-tetraisopropyl-1-(1-butylamidinato)guanidinato$\left.\kappa^{3} N^{1}, N^{2}: N^{2}\right]$ bis [(tetrahydrofuran)lithium]

Crystal data

$\left.\left[\mathrm{Li}_{2} \mathrm{C}_{18} \mathrm{H}_{37} \mathrm{~N}_{4}\right)_{2}\left(\mathrm{C}_{4} \mathrm{H}_{8} \mathrm{O}\right)_{2}\right]$

$M_{r}=777.11$

Monoclinic, $P 2_{1} / c$

$a=9.93297(7) \AA$

$b=13.7239$ (1) $\AA$

$c=18.07940(13) \AA$

$\beta=92.8380(6)^{\circ}$

$V=2461.54(3) \AA^{3}$

$Z=2$

$F(000)=864$

$D_{\mathrm{x}}=1.048 \mathrm{Mg} \mathrm{m}^{-3}$

$\mathrm{Cu} K \alpha$ radiation, $\lambda=1.54184 \AA$

Cell parameters from 18888 reflections

$\theta=3.2-75.6^{\circ}$

$\mu=0.49 \mathrm{~mm}^{-1}$

$T=100 \mathrm{~K}$

Plate, colorless

$0.18 \times 0.12 \times 0.04 \mathrm{~mm}$

Data collection

Agilent Xcalibur, Atlas, Nova diffractometer

Radiation source: Nova $(\mathrm{Cu}) \mathrm{X}$-ray Source Detector resolution: 10.3543 pixels $\mathrm{mm}^{-1}$

$\omega$ scans

Absorption correction: multi-scan

(CrysAlisPro; Agilent, 2011)

$T_{\min }=0.891, T_{\max }=1.000$

30246 measured reflections

5126 independent reflections

4657 reflections with $I>2 \sigma(I)$

$R_{\text {int }}=0.027$

$\theta_{\text {max }}=75.8^{\circ}, \theta_{\min }=4.1^{\circ}$

$h=-12 \rightarrow 11$

$k=-16 \rightarrow 17$

$l=-17 \rightarrow 22$ 


\section{Refinement}

Refinement on $F^{2}$

Least-squares matrix: full

$R\left[F^{2}>2 \sigma\left(F^{2}\right)\right]=0.036$

$w R\left(F^{2}\right)=0.096$

$S=1.05$

5126 reflections

263 parameters

0 restraints

Primary atom site location: structure-invariant direct methods

Secondary atom site location: difference Fourier map
Hydrogen site location: inferred from neighbouring sites

$\mathrm{H}$-atom parameters constrained

$w=1 /\left[\sigma^{2}\left(F_{\mathrm{o}}^{2}\right)+(0.0474 P)^{2}+0.6355 P\right]$

where $P=\left(F_{\mathrm{o}}^{2}+2 F_{\mathrm{c}}{ }^{2}\right) / 3$

$(\Delta / \sigma)_{\max }=0.001$

$\Delta \rho_{\max }=0.31 \mathrm{e} \AA^{-3}$

$\Delta \rho_{\min }=-0.21$ e $\AA^{-3}$

Extinction correction: SHELXL2016

(Sheldrick, 2015),

$\mathrm{Fc}^{*}=\mathrm{kFc}\left[1+0.001 \mathrm{xFc}^{2} \lambda^{3} / \sin (2 \theta)\right]^{-1 / 4}$

Extinction coefficient: 0.00067 (16)

\section{Special details}

Geometry. All esds (except the esd in the dihedral angle between two 1.s. planes) are estimated using the full covariance matrix. The cell esds are taken into account individually in the estimation of esds in distances, angles and torsion angles; correlations between esds in cell parameters are only used when they are defined by crystal symmetry. An approximate (isotropic) treatment of cell esds is used for estimating esds involving l.s. planes.

Fractional atomic coordinates and isotropic or equivalent isotropic displacement parameters $\left(\AA^{2}\right)$

\begin{tabular}{lllll}
\hline & $x$ & $y$ & $z$ & $U_{\text {iso }} * / U_{\text {eq }}$ \\
\hline C1 & $1.13747(9)$ & $0.49827(6)$ & $0.11713(5)$ & $0.01508(18)$ \\
C2 & $0.98312(9)$ & $0.49701(7)$ & $0.21715(5)$ & $0.0199(2)$ \\
H2 & 1.067737 & 0.513701 & 0.246717 & $0.024^{*}$ \\
C3 & $0.92362(11)$ & $0.40357(8)$ & $0.24810(6)$ & $0.0286(2)$ \\
H3A & 0.896871 & 0.415184 & 0.298801 & $0.034^{*}$ \\
H3B & 0.991346 & 0.351631 & 0.248263 & $0.034^{*}$ \\
H3C & 0.844533 & 0.384025 & 0.217059 & $0.034^{*}$ \\
C4 & $0.88214(10)$ & $0.58050(8)$ & $0.22330(6)$ & $0.0269(2)$ \\
H4A & 0.866222 & 0.592480 & 0.275587 & $0.032^{*}$ \\
H4B & 0.796995 & 0.562914 & 0.196968 & $0.032^{*}$ \\
H4C & 0.918553 & 0.639559 & 0.201291 & $0.032^{*}$ \\
C5 & $1.28751(9)$ & $0.48998(7)$ & $0.01575(5)$ & $0.01694(19)$ \\
H5 & 1.355755 & 0.487672 & 0.058274 & $0.020^{*}$ \\
C6 & $1.28738(10)$ & $0.39198(7)$ & $-0.02470(5)$ & $0.0216(2)$ \\
H6A & 1.376825 & 0.379957 & -0.043384 & $0.026^{*}$ \\
H6B & 1.220220 & 0.393392 & -0.066259 & $0.026^{*}$ \\
H6C & 1.265025 & 0.339863 & 0.009657 & $0.026^{*}$ \\
C7 & $1.32471(9)$ & $0.57220(7)$ & $-0.03661(5)$ & $0.0223(2)$ \\
H7A & 1.415937 & 0.561386 & -0.053232 & $0.027^{*}$ \\
H7B & 1.321415 & 0.634711 & -0.010560 & $0.027^{*}$ \\
H7C & 1.260605 & 0.573175 & -0.079593 & $0.027^{*}$ \\
C8 & $1.27924(9)$ & $0.42776(7)$ & $0.21963(5)$ & $0.01661(18)$ \\
C9 & $1.32067(9)$ & $0.59902(7)$ & $0.17680(5)$ & $0.01914(19)$ \\
H9 & 1.308813 & 0.631572 & 0.127416 & $0.023^{*}$ \\
C10 & $1.25452(10)$ & $0.66563(7)$ & $0.23194(6)$ & $0.0250(2)$ \\
H10A & 1.301263 & 0.728531 & 0.233932 & $0.030^{*}$ \\
H10B & 1.260085 & 0.635471 & 0.281130 & $0.030^{*}$ \\
\end{tabular}




\begin{tabular}{|c|c|c|c|c|}
\hline $\mathrm{H} 10 \mathrm{C}$ & 1.159716 & 0.675682 & 0.216188 & $0.030^{*}$ \\
\hline $\mathrm{C} 11$ & $1.47245(9)$ & $0.59045(7)$ & $0.19329(5)$ & $0.0230(2)$ \\
\hline H11A & 1.515323 & 0.653447 & 0.184560 & $0.028^{*}$ \\
\hline H11B & 1.509569 & 0.541135 & 0.160805 & $0.028^{*}$ \\
\hline $\mathrm{H} 11 \mathrm{C}$ & 1.489873 & 0.571267 & 0.245094 & $0.028^{*}$ \\
\hline $\mathrm{C} 12$ & $1.37114(11)$ & $0.37049(7)$ & $0.33586(5)$ & $0.0240(2)$ \\
\hline H12 & 1.353554 & 0.305706 & 0.311966 & $0.029^{*}$ \\
\hline $\mathrm{C} 13$ & $1.28767(12)$ & $0.38011(9)$ & $0.40405(6)$ & $0.0340(3)$ \\
\hline $\mathrm{H} 13 \mathrm{~A}$ & 1.312206 & 0.327951 & 0.439204 & $0.041^{*}$ \\
\hline H13B & 1.191678 & 0.375085 & 0.389225 & $0.041 *$ \\
\hline $\mathrm{H} 13 \mathrm{C}$ & 1.305491 & 0.443472 & 0.427535 & $0.041 *$ \\
\hline $\mathrm{C} 14$ & $1.52124(11)$ & $0.38091(9)$ & $0.35630(6)$ & $0.0310(2)$ \\
\hline $\mathrm{H} 14 \mathrm{~A}$ & 1.549829 & 0.328604 & 0.390479 & $0.037 *$ \\
\hline H14B & 1.538082 & 0.444157 & 0.380172 & $0.037^{*}$ \\
\hline $\mathrm{H} 14 \mathrm{C}$ & 1.572284 & 0.376680 & 0.311393 & $0.037 *$ \\
\hline $\mathrm{C} 15$ & $1.25689(9)$ & $0.32623(7)$ & $0.18708(5)$ & $0.01830(19)$ \\
\hline $\mathrm{H} 15 \mathrm{~A}$ & 1.186367 & 0.329059 & 0.146475 & $0.022 *$ \\
\hline H15B & 1.225324 & 0.281858 & 0.225761 & $0.022 *$ \\
\hline $\mathrm{C} 16$ & $1.38830(9)$ & $0.28650(7)$ & $0.15713(5)$ & 0.01958 (19) \\
\hline H16A & 1.424067 & 0.334657 & 0.122370 & $0.024 *$ \\
\hline H16B & 1.455792 & 0.278751 & 0.198869 & $0.024 *$ \\
\hline $\mathrm{C} 17$ & $1.37014(10)$ & $0.18882(7)$ & $0.11733(5)$ & $0.0215(2)$ \\
\hline H17A & 1.346490 & 0.138322 & 0.153569 & $0.026^{*}$ \\
\hline H17B & 1.294556 & 0.193946 & 0.079734 & $0.026^{*}$ \\
\hline $\mathrm{C} 18$ & $1.49719(11)$ & $0.15769(8)$ & $0.07957(6)$ & $0.0281(2)$ \\
\hline $\mathrm{H} 18 \mathrm{~A}$ & 1.479126 & 0.097653 & 0.051461 & $0.034 *$ \\
\hline H18B & 1.569961 & 0.146142 & 0.117146 & $0.034 *$ \\
\hline $\mathrm{H} 18 \mathrm{C}$ & 1.524114 & 0.209274 & 0.045858 & $0.034 *$ \\
\hline $\mathrm{C} 19$ & $0.94341(14)$ & $0.23713(9)$ & $-0.03529(6)$ & $0.0357(3)$ \\
\hline H19A & 1.029322 & 0.232716 & -0.060603 & $0.043^{*}$ \\
\hline H19B & 0.876981 & 0.272617 & -0.067824 & $0.043^{*}$ \\
\hline $\mathrm{C} 20$ & $0.89174(18)$ & $0.13655(11)$ & $-0.01791(8)$ & $0.0536(4)$ \\
\hline $\mathrm{H} 20 \mathrm{~A}$ & 0.932479 & 0.086723 & -0.049483 & $0.064 *$ \\
\hline H20B & 0.792403 & 0.133407 & -0.025275 & $0.064 *$ \\
\hline $\mathrm{C} 21$ & $0.93514(17)$ & $0.12190(9)$ & $0.06268(8)$ & $0.0476(3)$ \\
\hline $\mathrm{H} 21 \mathrm{~A}$ & 0.874625 & 0.075971 & 0.087114 & $0.057^{*}$ \\
\hline $\mathrm{H} 21 \mathrm{~B}$ & 1.028931 & 0.097574 & 0.068212 & $0.057^{*}$ \\
\hline $\mathrm{C} 22$ & $0.92342(12)$ & $0.22318(8)$ & $0.09337(6)$ & $0.0292(2)$ \\
\hline $\mathrm{H} 22 \mathrm{~A}$ & 0.829573 & 0.236969 & 0.106203 & $0.035^{*}$ \\
\hline $\mathrm{H} 22 \mathrm{~B}$ & 0.983554 & 0.231476 & 0.138250 & $0.035^{*}$ \\
\hline LI & $0.96597(16)$ & $0.42919(12)$ & $0.03809(9)$ & $0.0200(3)$ \\
\hline N1 & $1.01480(8)$ & $0.48338(6)$ & $0.13965(4)$ & $0.01756(17)$ \\
\hline N2 & $1.15401(7)$ & $0.50792(5)$ & $0.04440(4)$ & $0.01578(16)$ \\
\hline N3 & $1.25379(7)$ & $0.50292(5)$ & $0.16898(4)$ & $0.01575(16)$ \\
\hline N4 & $1.32979(8)$ & $0.44822(6)$ & $0.28432(4)$ & 0.01977 (17) \\
\hline $\mathrm{O}$ & $0.96357(7)$ & $0.28667(5)$ & $0.03486(4)$ & $0.02535(17)$ \\
\hline
\end{tabular}


Atomic displacement parameters $\left(\AA^{2}\right)$

\begin{tabular}{|c|c|c|c|c|c|c|}
\hline & $U^{11}$ & $U^{22}$ & $U^{33}$ & $U^{12}$ & $U^{13}$ & $U^{23}$ \\
\hline $\mathrm{C} 1$ & $0.0155(4)$ & $0.0136(4)$ & $0.0160(4)$ & 0.0008 & -0.0011 & -0.0001 \\
\hline $\mathrm{C} 2$ & $0.0186(4)$ & $0.0254(5)$ & $0.0159(4)$ & $-0.0003(4)$ & 0.0025 & -0.0002 \\
\hline $\mathrm{C} 3$ & $0.0315(5)$ & $0.0328(6)$ & $0.0221(5)$ & $-0.0046(4)$ & 0.0078 & $0.0036(4)$ \\
\hline $\mathrm{C} 4$ & $0.0244(5)$ & $0.0347(6)$ & $0.0219(5)$ & $0.0067(4)$ & 0.0039 (4) & -0.0025 \\
\hline $\mathrm{C} 5$ & $0.0141(4)$ & $0.0205(4)$ & $0.0162(4)$ & 0.0009 & 0.0009 & 0.0013 \\
\hline C6 & $0.0232(5)$ & $0.0234(5)$ & $0.0184(4)$ & 0.0025 & 0.0028 & -0.0005 (4) \\
\hline $\mathrm{C} 7$ & $0.0182(4)$ & $0.0249(5)$ & $0.0241(5)$ & $-0.0003(4)$ & $0.0052(3)$ & $0.0041(4)$ \\
\hline $\mathrm{C} 8$ & $0.0154(4)$ & $0.0174(4)$ & $0.0171(4)$ & $0.0004(3)$ & $0.0013(3)$ & 0.0010 \\
\hline C9 & $0.0202(4)$ & $0.0168(4)$ & $0.0202(4)$ & $-0.0028(3)$ & $-0.0013(3)$ & 0.0001 \\
\hline $\mathrm{C} 10$ & $0.0253(5)$ & $0.0203(5)$ & $0.0293(5)$ & $0.0001(4)$ & $0.0005(4)$ & $-0.0046(4)$ \\
\hline C11 & 0.0198 & $0.0256(5)$ & $0.0235(5)$ & $-0.0054(4)$ & $0.0005(4)$ & $-0.0033(4)$ \\
\hline $\mathrm{C} 12$ & $0.0322(5)$ & $0.0203(5)$ & $0.0189(5)$ & $0.0034(4)$ & $-0.0057(4)$ & $0.0021(4)$ \\
\hline C13 & $0.0382(6)$ & $0.0385(6)$ & $0.0252(5)$ & $0.0038(5)$ & $0.0018(4)$ & $0.0124(5)$ \\
\hline $\mathrm{C} 14$ & $0.0314(6)$ & $0.0336(6)$ & $0.0272(5)$ & $0.0099(4)$ & -0.0063 & $-0.0002(4)$ \\
\hline $\mathrm{C} 15$ & $0.0193(4)$ & $0.0166(4)$ & $0.0188(4)$ & -0.0008 & -0.0011 & 0.0009 \\
\hline C16 & 0.0205 & $0.0185(4)$ & $0.0195(4)$ & 0.0008 & -0.0012 & -0.0001 \\
\hline $\mathrm{C} 17$ & $0.0265(5)$ & $0.0183(4)$ & $0.0196(4)$ & $0.0012(4)$ & 0.0000 & $0.0004(3)$ \\
\hline $\mathrm{C} 18$ & $0.0325(5)$ & $0.0280(5)$ & $0.0239(5)$ & $0.0055(4)$ & 0.0025 & -0.0028 (4) \\
\hline C19 & $0.0500(7)$ & $0.0284(6)$ & $0.0290(6)$ & $0.0071(5)$ & $0.0045(5)$ & -0.0049 (4) \\
\hline $\mathrm{C} 20$ & $0.0773(11)$ & $0.0365(7)$ & $0.0462(8)$ & $-0.0150(7)$ & $-0.0042(7)$ & $-0.0097(6)$ \\
\hline $\mathrm{C} 21$ & $0.0688(9)$ & $0.0217(6)$ & $0.0518(8)$ & $-0.0069(6)$ & $-0.0025(7)$ & $0.0040(5)$ \\
\hline $\mathrm{C} 22$ & $0.0346(6)$ & $0.0232(5)$ & $0.0294(5)$ & $-0.0059(4)$ & -0.0020 & $0.0076(4)$ \\
\hline LI & $0.0200(7)$ & $0.0195(8)$ & $0.0203(8)$ & $-0.0011(6)$ & $-0.0004(6)$ & $0.0019(6)$ \\
\hline N1 & $0.0161(4)$ & $0.0217(4)$ & $0.0150(4)$ & -0.0003 & $0.0017(3)$ & 0.0006 \\
\hline $\mathrm{N} 2$ & 0.0134 & $0.0188(4)$ & $0.0152(4)$ & 0.0003 & 0.0010 & 0.0008 \\
\hline N3 & $0.0163(4)$ & $0.0153(4)$ & $0.0154(4)$ & $-0.0014(3)$ & -0.0019 & 0.0008 \\
\hline N4 & $0.0234(4)$ & $0.0190(4)$ & $0.0166(4)$ & 0.0018 & $-0.0016(3)$ & 0.0013 \\
\hline $\mathrm{O}$ & $0.0318(4)$ & 0.0186 & $0.0258(4)$ & -0.0010 & $0.0031(3)$ & 0.0021 \\
\hline
\end{tabular}

Geometric parameters $\left(A,{ }^{\circ}\right)$

\begin{tabular}{|c|c|c|c|}
\hline $\mathrm{C} 1-\mathrm{N} 1$ & $1.3197(12)$ & $\mathrm{C} 12-\mathrm{C} 13$ & $1.5251(15)$ \\
\hline $\mathrm{C} 1-\mathrm{N} 2$ & $1.3396(11)$ & $\mathrm{C} 12-\mathrm{H} 12$ & 1.0000 \\
\hline $\mathrm{C} 1-\mathrm{N} 3$ & $1.4528(11)$ & $\mathrm{C} 13-\mathrm{H} 13 \mathrm{~A}$ & 0.9800 \\
\hline $\mathrm{C} 1-\mathrm{LI}$ & $2.3667(18)$ & $\mathrm{C} 13-\mathrm{H} 13 \mathrm{~B}$ & 0.9800 \\
\hline $\mathrm{C} 2-\mathrm{N} 1$ & $1.4633(11)$ & $\mathrm{C} 13-\mathrm{H} 13 \mathrm{C}$ & 0.9800 \\
\hline $\mathrm{C} 2-\mathrm{C} 3$ & $1.5296(14)$ & $\mathrm{C} 14-\mathrm{H} 14 \mathrm{~A}$ & 0.9800 \\
\hline $\mathrm{C} 2-\mathrm{C} 4$ & $1.5304(13)$ & $\mathrm{C} 14-\mathrm{H} 14 \mathrm{~B}$ & 0.9800 \\
\hline $\mathrm{C} 2-\mathrm{H} 2$ & 1.0000 & $\mathrm{C} 14-\mathrm{H} 14 \mathrm{C}$ & 0.9800 \\
\hline $\mathrm{C} 3-\mathrm{H} 3 \mathrm{~A}$ & 0.9800 & $\mathrm{C} 15-\mathrm{C} 16$ & $1.5376(13)$ \\
\hline $\mathrm{C} 3-\mathrm{H} 3 \mathrm{~B}$ & 0.9800 & $\mathrm{C} 15-\mathrm{H} 15 \mathrm{~A}$ & 0.9900 \\
\hline $\mathrm{C} 3-\mathrm{H} 3 \mathrm{C}$ & 0.9800 & $\mathrm{C} 15-\mathrm{H} 15 \mathrm{~B}$ & 0.9900 \\
\hline $\mathrm{C} 4-\mathrm{H} 4 \mathrm{~A}$ & 0.9800 & $\mathrm{C} 16-\mathrm{C} 17$ & $1.5279(13)$ \\
\hline $\mathrm{C} 4-\mathrm{H} 4 \mathrm{~B}$ & 0.9800 & $\mathrm{C} 16-\mathrm{H} 16 \mathrm{~A}$ & 0.9900 \\
\hline $\mathrm{C} 4-\mathrm{H} 4 \mathrm{C}$ & 0.9800 & $\mathrm{C} 16-\mathrm{H} 16 \mathrm{~B}$ & 0.9900 \\
\hline
\end{tabular}




\begin{tabular}{|c|c|c|c|}
\hline $\mathrm{C} 5-\mathrm{N} 2$ & $1.4681(11)$ & $\mathrm{C} 17-\mathrm{C} 18$ & $1.5252(14)$ \\
\hline $\mathrm{C} 5-\mathrm{C} 7$ & $1.5299(13)$ & C17-H17A & 0.9900 \\
\hline $\mathrm{C} 5-\mathrm{C} 6$ & $1.5309(13)$ & $\mathrm{C} 17-\mathrm{H} 17 \mathrm{~B}$ & 0.9900 \\
\hline $\mathrm{C} 5-\mathrm{H} 5$ & 1.0000 & $\mathrm{C} 18-\mathrm{H} 18 \mathrm{~A}$ & 0.9800 \\
\hline C6-H6A & 0.9800 & $\mathrm{C} 18-\mathrm{H} 18 \mathrm{~B}$ & 0.9800 \\
\hline C6-H6B & 0.9800 & $\mathrm{C} 18-\mathrm{H} 18 \mathrm{C}$ & 0.9800 \\
\hline C6- $\mathrm{H} 6 \mathrm{C}$ & 0.9800 & $\mathrm{C} 19-\mathrm{O}$ & $1.4441(13)$ \\
\hline C7-H7A & 0.9800 & $\mathrm{C} 19-\mathrm{C} 20$ & $1.5111(18)$ \\
\hline C7-H7B & 0.9800 & C19-H19A & 0.9900 \\
\hline $\mathrm{C} 7-\mathrm{H} 7 \mathrm{C}$ & 0.9800 & C19-H19B & 0.9900 \\
\hline $\mathrm{C} 8-\mathrm{N} 4$ & $1.2808(12)$ & $\mathrm{C} 20-\mathrm{C} 21$ & $1.512(2)$ \\
\hline $\mathrm{C} 8-\mathrm{N} 3$ & $1.3940(11)$ & $\mathrm{C} 20-\mathrm{H} 20 \mathrm{~A}$ & 0.9900 \\
\hline $\mathrm{C} 8-\mathrm{C} 15$ & $1.5245(12)$ & $\mathrm{C} 20-\mathrm{H} 20 \mathrm{~B}$ & 0.9900 \\
\hline $\mathrm{C} 9-\mathrm{N} 3$ & $1.4804(11)$ & $\mathrm{C} 21-\mathrm{C} 22$ & $1.5033(17)$ \\
\hline $\mathrm{C} 9-\mathrm{C} 10$ & $1.5251(13)$ & $\mathrm{C} 21-\mathrm{H} 21 \mathrm{~A}$ & 0.9900 \\
\hline $\mathrm{C} 9-\mathrm{C} 11$ & $1.5268(13)$ & $\mathrm{C} 21-\mathrm{H} 21 \mathrm{~B}$ & 0.9900 \\
\hline C9-H9 & 1.0000 & $\mathrm{C} 22-\mathrm{O}$ & $1.4419(12)$ \\
\hline $\mathrm{C} 10-\mathrm{H} 10 \mathrm{~A}$ & 0.9800 & $\mathrm{C} 22-\mathrm{H} 22 \mathrm{~A}$ & 0.9900 \\
\hline $\mathrm{C} 10-\mathrm{H} 10 \mathrm{~B}$ & 0.9800 & $\mathrm{C} 22-\mathrm{H} 22 \mathrm{~B}$ & 0.9900 \\
\hline $\mathrm{C} 10-\mathrm{H} 10 \mathrm{C}$ & 0.9800 & $\mathrm{LI}-\mathrm{O}$ & $1.9569(18)$ \\
\hline $\mathrm{C} 11-\mathrm{H} 11 \mathrm{~A}$ & 0.9800 & $\mathrm{LI}-\mathrm{N} 1$ & $2.0177(18)$ \\
\hline $\mathrm{C} 11-\mathrm{H} 11 \mathrm{~B}$ & 0.9800 & $\mathrm{LI}-\mathrm{N} 2^{\mathrm{i}}$ & $2.0528(17)$ \\
\hline $\mathrm{C} 11-\mathrm{H} 11 \mathrm{C}$ & 0.9800 & $\mathrm{LI}-\mathrm{N} 2$ & $2.1559(17)$ \\
\hline $\mathrm{C} 12-\mathrm{N} 4$ & $1.4619(12)$ & $\mathrm{LI}-\mathrm{LI}^{\mathrm{i}}$ & $2.495(3)$ \\
\hline $\mathrm{C} 12-\mathrm{C} 14$ & $1.5250(15)$ & $\mathrm{N} 2-\mathrm{LI}^{\mathrm{i}}$ & $2.0528(17)$ \\
\hline $\mathrm{N} 1-\mathrm{C} 1-\mathrm{N} 2$ & $118.56(8)$ & $\mathrm{H} 14 \mathrm{~B}-\mathrm{C} 14-\mathrm{H} 14 \mathrm{C}$ & 109.5 \\
\hline $\mathrm{N} 1-\mathrm{C} 1-\mathrm{N} 3$ & $121.64(8)$ & $\mathrm{C} 8-\mathrm{C} 15-\mathrm{C} 16$ & $110.49(7)$ \\
\hline $\mathrm{N} 2-\mathrm{C} 1-\mathrm{N} 3$ & $119.79(8)$ & $\mathrm{C} 8-\mathrm{C} 15-\mathrm{H} 15 \mathrm{~A}$ & 109.6 \\
\hline $\mathrm{N} 1-\mathrm{C} 1-\mathrm{LI}$ & $58.42(6)$ & $\mathrm{C} 16-\mathrm{C} 15-\mathrm{H} 15 \mathrm{~A}$ & 109.6 \\
\hline $\mathrm{N} 2-\mathrm{C} 1-\mathrm{LI}$ & $64.32(6)$ & $\mathrm{C} 8-\mathrm{C} 15-\mathrm{H} 15 \mathrm{~B}$ & 109.6 \\
\hline $\mathrm{N} 3-\mathrm{C} 1-\mathrm{LI}$ & $158.88(7)$ & $\mathrm{C} 16-\mathrm{C} 15-\mathrm{H} 15 \mathrm{~B}$ & 109.6 \\
\hline $\mathrm{N} 1-\mathrm{C} 2-\mathrm{C} 3$ & $110.39(8)$ & $\mathrm{H} 15 \mathrm{~A}-\mathrm{C} 15-\mathrm{H} 15 \mathrm{~B}$ & 108.1 \\
\hline $\mathrm{N} 1-\mathrm{C} 2-\mathrm{C} 4$ & $109.76(8)$ & $\mathrm{C} 17-\mathrm{C} 16-\mathrm{C} 15$ & $113.24(8)$ \\
\hline $\mathrm{C} 3-\mathrm{C} 2-\mathrm{C} 4$ & $109.55(8)$ & $\mathrm{C} 17-\mathrm{C} 16-\mathrm{H} 16 \mathrm{~A}$ & 108.9 \\
\hline $\mathrm{N} 1-\mathrm{C} 2-\mathrm{H} 2$ & 109.0 & $\mathrm{C} 15-\mathrm{C} 16-\mathrm{H} 16 \mathrm{~A}$ & 108.9 \\
\hline $\mathrm{C} 3-\mathrm{C} 2-\mathrm{H} 2$ & 109.0 & $\mathrm{C} 17-\mathrm{C} 16-\mathrm{H} 16 \mathrm{~B}$ & 108.9 \\
\hline $\mathrm{C} 4-\mathrm{C} 2-\mathrm{H} 2$ & 109.0 & $\mathrm{C} 15-\mathrm{C} 16-\mathrm{H} 16 \mathrm{~B}$ & 108.9 \\
\hline $\mathrm{C} 2-\mathrm{C} 3-\mathrm{H} 3 \mathrm{~A}$ & 109.5 & $\mathrm{H} 16 \mathrm{~A}-\mathrm{C} 16-\mathrm{H} 16 \mathrm{~B}$ & 107.7 \\
\hline $\mathrm{C} 2-\mathrm{C} 3-\mathrm{H} 3 \mathrm{~B}$ & 109.5 & $\mathrm{C} 18-\mathrm{C} 17-\mathrm{C} 16$ & $112.06(8)$ \\
\hline $\mathrm{H} 3 \mathrm{~A}-\mathrm{C} 3-\mathrm{H} 3 \mathrm{~B}$ & 109.5 & $\mathrm{C} 18-\mathrm{C} 17-\mathrm{H} 17 \mathrm{~A}$ & 109.2 \\
\hline $\mathrm{C} 2-\mathrm{C} 3-\mathrm{H} 3 \mathrm{C}$ & 109.5 & $\mathrm{C} 16-\mathrm{C} 17-\mathrm{H} 17 \mathrm{~A}$ & 109.2 \\
\hline $\mathrm{H} 3 \mathrm{~A}-\mathrm{C} 3-\mathrm{H} 3 \mathrm{C}$ & 109.5 & $\mathrm{C} 18-\mathrm{C} 17-\mathrm{H} 17 \mathrm{~B}$ & 109.2 \\
\hline $\mathrm{H} 3 \mathrm{~B}-\mathrm{C} 3-\mathrm{H} 3 \mathrm{C}$ & 109.5 & $\mathrm{C} 16-\mathrm{C} 17-\mathrm{H} 17 \mathrm{~B}$ & 109.2 \\
\hline $\mathrm{C} 2-\mathrm{C} 4-\mathrm{H} 4 \mathrm{~A}$ & 109.5 & $\mathrm{H} 17 \mathrm{~A}-\mathrm{C} 17-\mathrm{H} 17 \mathrm{~B}$ & 107.9 \\
\hline $\mathrm{C} 2-\mathrm{C} 4-\mathrm{H} 4 \mathrm{~B}$ & 109.5 & $\mathrm{C} 17-\mathrm{C} 18-\mathrm{H} 18 \mathrm{~A}$ & 109.5 \\
\hline $\mathrm{H} 4 \mathrm{~A}-\mathrm{C} 4-\mathrm{H} 4 \mathrm{~B}$ & 109.5 & $\mathrm{C} 17-\mathrm{C} 18-\mathrm{H} 18 \mathrm{~B}$ & 109.5 \\
\hline $\mathrm{C} 2-\mathrm{C} 4-\mathrm{H} 4 \mathrm{C}$ & 109.5 & $\mathrm{H} 18 \mathrm{~A}-\mathrm{C} 18-\mathrm{H} 18 \mathrm{~B}$ & 109.5 \\
\hline
\end{tabular}




\begin{tabular}{|c|c|c|c|}
\hline $\mathrm{H} 4 \mathrm{~A}-\mathrm{C} 4-\mathrm{H} 4 \mathrm{C}$ & 109.5 & $\mathrm{C} 17-\mathrm{C} 18-\mathrm{H} 18 \mathrm{C}$ & 109.5 \\
\hline $\mathrm{H} 4 \mathrm{~B}-\mathrm{C} 4-\mathrm{H} 4 \mathrm{C}$ & 109.5 & $\mathrm{H} 18 \mathrm{~A}-\mathrm{C} 18-\mathrm{H} 18 \mathrm{C}$ & 109.5 \\
\hline $\mathrm{N} 2-\mathrm{C} 5-\mathrm{C} 7$ & $110.17(7)$ & $\mathrm{H} 18 \mathrm{~B}-\mathrm{C} 18-\mathrm{H} 18 \mathrm{C}$ & 109.5 \\
\hline $\mathrm{N} 2-\mathrm{C} 5-\mathrm{C} 6$ & $109.66(7)$ & $\mathrm{O}-\mathrm{C} 19-\mathrm{C} 20$ & $106.34(10)$ \\
\hline $\mathrm{C} 7-\mathrm{C} 5-\mathrm{C} 6$ & $110.30(8)$ & $\mathrm{O}-\mathrm{C} 19-\mathrm{H} 19 \mathrm{~A}$ & 110.5 \\
\hline $\mathrm{N} 2-\mathrm{C} 5-\mathrm{H} 5$ & 108.9 & $\mathrm{C} 20-\mathrm{C} 19-\mathrm{H} 19 \mathrm{~A}$ & 110.5 \\
\hline $\mathrm{C} 7-\mathrm{C} 5-\mathrm{H} 5$ & 108.9 & $\mathrm{O}-\mathrm{C} 19-\mathrm{H} 19 \mathrm{~B}$ & 110.5 \\
\hline $\mathrm{C} 6-\mathrm{C} 5-\mathrm{H} 5$ & 108.9 & $\mathrm{C} 20-\mathrm{C} 19-\mathrm{H} 19 \mathrm{~B}$ & 110.5 \\
\hline $\mathrm{C} 5-\mathrm{C} 6-\mathrm{H} 6 \mathrm{~A}$ & 109.5 & $\mathrm{H} 19 \mathrm{~A}-\mathrm{C} 19-\mathrm{H} 19 \mathrm{~B}$ & 108.7 \\
\hline $\mathrm{C} 5-\mathrm{C} 6-\mathrm{H} 6 \mathrm{~B}$ & 109.5 & $\mathrm{C} 19-\mathrm{C} 20-\mathrm{C} 21$ & $103.78(11)$ \\
\hline $\mathrm{H} 6 \mathrm{~A}-\mathrm{C} 6-\mathrm{H} 6 \mathrm{~B}$ & 109.5 & $\mathrm{C} 19-\mathrm{C} 20-\mathrm{H} 20 \mathrm{~A}$ & 111.0 \\
\hline $\mathrm{C} 5-\mathrm{C} 6-\mathrm{H} 6 \mathrm{C}$ & 109.5 & $\mathrm{C} 21-\mathrm{C} 20-\mathrm{H} 20 \mathrm{~A}$ & 111.0 \\
\hline $\mathrm{H} 6 \mathrm{~A}-\mathrm{C} 6-\mathrm{H} 6 \mathrm{C}$ & 109.5 & $\mathrm{C} 19-\mathrm{C} 20-\mathrm{H} 20 \mathrm{~B}$ & 111.0 \\
\hline $\mathrm{H} 6 \mathrm{~B}-\mathrm{C} 6-\mathrm{H} 6 \mathrm{C}$ & 109.5 & $\mathrm{C} 21-\mathrm{C} 20-\mathrm{H} 20 \mathrm{~B}$ & 111.0 \\
\hline $\mathrm{C} 5-\mathrm{C} 7-\mathrm{H} 7 \mathrm{~A}$ & 109.5 & $\mathrm{H} 20 \mathrm{~A}-\mathrm{C} 20-\mathrm{H} 20 \mathrm{~B}$ & 109.0 \\
\hline $\mathrm{C} 5-\mathrm{C} 7-\mathrm{H} 7 \mathrm{~B}$ & 109.5 & $\mathrm{C} 22-\mathrm{C} 21-\mathrm{C} 20$ & $102.07(11)$ \\
\hline $\mathrm{H} 7 \mathrm{~A}-\mathrm{C} 7-\mathrm{H} 7 \mathrm{~B}$ & 109.5 & $\mathrm{C} 22-\mathrm{C} 21-\mathrm{H} 21 \mathrm{~A}$ & 111.4 \\
\hline $\mathrm{C} 5-\mathrm{C} 7-\mathrm{H} 7 \mathrm{C}$ & 109.5 & $\mathrm{C} 20-\mathrm{C} 21-\mathrm{H} 21 \mathrm{~A}$ & 111.4 \\
\hline $\mathrm{H} 7 \mathrm{~A}-\mathrm{C} 7-\mathrm{H} 7 \mathrm{C}$ & 109.5 & $\mathrm{C} 22-\mathrm{C} 21-\mathrm{H} 21 \mathrm{~B}$ & 111.4 \\
\hline $\mathrm{H} 7 \mathrm{~B}-\mathrm{C} 7-\mathrm{H} 7 \mathrm{C}$ & 109.5 & $\mathrm{C} 20-\mathrm{C} 21-\mathrm{H} 21 \mathrm{~B}$ & 111.4 \\
\hline $\mathrm{N} 4-\mathrm{C} 8-\mathrm{N} 3$ & $119.19(8)$ & $\mathrm{H} 21 \mathrm{~A}-\mathrm{C} 21-\mathrm{H} 21 \mathrm{~B}$ & 109.2 \\
\hline $\mathrm{N} 4-\mathrm{C} 8-\mathrm{C} 15$ & $126.58(8)$ & $\mathrm{O}-\mathrm{C} 22-\mathrm{C} 21$ & $104.99(9)$ \\
\hline $\mathrm{N} 3-\mathrm{C} 8-\mathrm{C} 15$ & $113.86(8)$ & $\mathrm{O}-\mathrm{C} 22-\mathrm{H} 22 \mathrm{~A}$ & 110.7 \\
\hline $\mathrm{N} 3-\mathrm{C} 9-\mathrm{C} 10$ & $112.98(8)$ & $\mathrm{C} 21-\mathrm{C} 22-\mathrm{H} 22 \mathrm{~A}$ & 110.7 \\
\hline $\mathrm{N} 3-\mathrm{C} 9-\mathrm{C} 11$ & $112.59(8)$ & $\mathrm{O}-\mathrm{C} 22-\mathrm{H} 22 \mathrm{~B}$ & 110.7 \\
\hline $\mathrm{C} 10-\mathrm{C} 9-\mathrm{C} 11$ & $111.82(8)$ & $\mathrm{C} 21-\mathrm{C} 22-\mathrm{H} 22 \mathrm{~B}$ & 110.7 \\
\hline $\mathrm{N} 3-\mathrm{C} 9-\mathrm{H} 9$ & 106.3 & $\mathrm{H} 22 \mathrm{~A}-\mathrm{C} 22-\mathrm{H} 22 \mathrm{~B}$ & 108.8 \\
\hline $\mathrm{C} 10-\mathrm{C} 9-\mathrm{H} 9$ & 106.3 & $\mathrm{O}-\mathrm{LI}-\mathrm{N} 1$ & $113.43(8)$ \\
\hline $\mathrm{C} 11-\mathrm{C} 9-\mathrm{H} 9$ & 106.3 & $\mathrm{O}-\mathrm{LI}-\mathrm{N} 2^{\mathrm{i}}$ & $113.13(8)$ \\
\hline $\mathrm{C} 9-\mathrm{C} 10-\mathrm{H} 10 \mathrm{~A}$ & 109.5 & $\mathrm{~N} 1-\mathrm{LI}-\mathrm{N} 2^{\mathrm{i}}$ & $127.65(9)$ \\
\hline $\mathrm{C} 9-\mathrm{C} 10-\mathrm{H} 10 \mathrm{~B}$ & 109.5 & $\mathrm{O}-\mathrm{LI}-\mathrm{N} 2$ & $120.78(8)$ \\
\hline $\mathrm{H} 10 \mathrm{~A}-\mathrm{C} 10-\mathrm{H} 10 \mathrm{~B}$ & 109.5 & $\mathrm{~N} 1-\mathrm{LI}-\mathrm{N} 2$ & $66.33(6)$ \\
\hline $\mathrm{C} 9-\mathrm{C} 10-\mathrm{H} 10 \mathrm{C}$ & 109.5 & $\mathrm{~N} 2 \mathrm{i}-\mathrm{LI}-\mathrm{N} 2$ & $107.34(7)$ \\
\hline $\mathrm{H} 10 \mathrm{~A}-\mathrm{C} 10-\mathrm{H} 10 \mathrm{C}$ & 109.5 & $\mathrm{O}-\mathrm{LI}-\mathrm{C} 1$ & $115.20(8)$ \\
\hline $\mathrm{H} 10 \mathrm{~B}-\mathrm{C} 10-\mathrm{H} 10 \mathrm{C}$ & 109.5 & $\mathrm{~N} 1-\mathrm{LI}-\mathrm{C} 1$ & $33.86(4)$ \\
\hline $\mathrm{C} 9-\mathrm{C} 11-\mathrm{H} 11 \mathrm{~A}$ & 109.5 & $\mathrm{~N} 2 \mathrm{i}-\mathrm{LI}-\mathrm{C} 1$ & $130.16(8)$ \\
\hline C9-C11-H11B & 109.5 & $\mathrm{~N} 2-\mathrm{LI}-\mathrm{C} 1$ & $34.06(4)$ \\
\hline $\mathrm{H} 11 \mathrm{~A}-\mathrm{C} 11-\mathrm{H} 11 \mathrm{~B}$ & 109.5 & $\mathrm{O}-\mathrm{LI}-\mathrm{LI}^{\mathrm{i}}$ & $139.94(12)$ \\
\hline $\mathrm{C} 9-\mathrm{C} 11-\mathrm{H} 11 \mathrm{C}$ & 109.5 & $\mathrm{~N} 1-\mathrm{LI}-\mathrm{LI}^{\mathrm{i}}$ & $98.96(9)$ \\
\hline $\mathrm{H} 11 \mathrm{~A}-\mathrm{C} 11-\mathrm{H} 11 \mathrm{C}$ & 109.5 & $\mathrm{~N} 22^{\mathrm{i}}-\mathrm{LI}-\mathrm{LI}^{\mathrm{i}}$ & $55.58(6)$ \\
\hline $\mathrm{H} 11 \mathrm{~B}-\mathrm{C} 11-\mathrm{H} 11 \mathrm{C}$ & 109.5 & $\mathrm{~N} 2-\mathrm{LI}-\mathrm{LI}^{\mathrm{i}}$ & $51.76(6)$ \\
\hline $\mathrm{N} 4-\mathrm{C} 12-\mathrm{C} 14$ & $109.05(8)$ & $\mathrm{C} 1-\mathrm{LI}-\mathrm{LI}^{\mathrm{i}}$ & $79.32(8)$ \\
\hline $\mathrm{N} 4-\mathrm{C} 12-\mathrm{C} 13$ & $107.78(8)$ & $\mathrm{C} 1-\mathrm{N} 1-\mathrm{C} 2$ & $121.42(8)$ \\
\hline $\mathrm{C} 14-\mathrm{C} 12-\mathrm{C} 13$ & $111.10(9)$ & $\mathrm{C} 1-\mathrm{N} 1-\mathrm{LI}$ & $87.72(7)$ \\
\hline $\mathrm{N} 4-\mathrm{C} 12-\mathrm{H} 12$ & 109.6 & $\mathrm{C} 2-\mathrm{N} 1-\mathrm{LI}$ & $149.90(8)$ \\
\hline $\mathrm{C} 14-\mathrm{C} 12-\mathrm{H} 12$ & 109.6 & $\mathrm{C} 1-\mathrm{N} 2-\mathrm{C} 5$ & $119.11(7)$ \\
\hline $\mathrm{C} 13-\mathrm{C} 12-\mathrm{H} 12$ & 109.6 & $\mathrm{C} 1-\mathrm{N} 2-\mathrm{LI}^{\mathrm{i}}$ & $131.28(8)$ \\
\hline $\mathrm{C} 12-\mathrm{C} 13-\mathrm{H} 13 \mathrm{~A}$ & 109.5 & $\mathrm{C} 5-\mathrm{N} 2-\mathrm{LI}^{\mathrm{i}}$ & $108.43(7)$ \\
\hline
\end{tabular}




\begin{tabular}{|c|c|c|c|}
\hline $\mathrm{C} 12-\mathrm{C} 13-\mathrm{H} 13 \mathrm{~B}$ & 109.5 & $\mathrm{C} 1-\mathrm{N} 2-\mathrm{LI}$ & $81.63(7)$ \\
\hline $\mathrm{H} 13 \mathrm{~A}-\mathrm{C} 13-\mathrm{H} 13 \mathrm{~B}$ & 109.5 & $\mathrm{C} 5-\mathrm{N} 2-\mathrm{LI}$ & $133.84(7)$ \\
\hline $\mathrm{C} 12-\mathrm{C} 13-\mathrm{H} 13 \mathrm{C}$ & 109.5 & $\mathrm{LI}-\mathrm{N} 2-\mathrm{LI}$ & $72.66(7)$ \\
\hline $\mathrm{H} 13 \mathrm{~A}-\mathrm{C} 13-\mathrm{H} 13 \mathrm{C}$ & 109.5 & $\mathrm{C} 8-\mathrm{N} 3-\mathrm{C} 1$ & $120.26(7)$ \\
\hline $\mathrm{H} 13 \mathrm{~B}-\mathrm{C} 13-\mathrm{H} 13 \mathrm{C}$ & 109.5 & $\mathrm{C} 8-\mathrm{N} 3-\mathrm{C} 9$ & $122.05(7)$ \\
\hline $\mathrm{C} 12-\mathrm{C} 14-\mathrm{H} 14 \mathrm{~A}$ & 109.5 & $\mathrm{C} 1-\mathrm{N} 3-\mathrm{C} 9$ & $116.07(7)$ \\
\hline $\mathrm{C} 12-\mathrm{C} 14-\mathrm{H} 14 \mathrm{~B}$ & 109.5 & $\mathrm{C} 8-\mathrm{N} 4-\mathrm{C} 12$ & $120.47(8)$ \\
\hline $\mathrm{H} 14 \mathrm{~A}-\mathrm{C} 14-\mathrm{H} 14 \mathrm{~B}$ & 109.5 & $\mathrm{C} 22-\mathrm{O}-\mathrm{C} 19$ & $109.17(8)$ \\
\hline $\mathrm{C} 12-\mathrm{C} 14-\mathrm{H} 14 \mathrm{C}$ & 109.5 & $\mathrm{C} 22-\mathrm{O}-\mathrm{LI}$ & $125.84(8)$ \\
\hline $\mathrm{H} 14 \mathrm{~A}-\mathrm{C} 14-\mathrm{H} 14 \mathrm{C}$ & 109.5 & $\mathrm{C} 19-\mathrm{O}-\mathrm{LI}$ & $119.85(8)$ \\
\hline $\mathrm{N} 4-\mathrm{C} 8-\mathrm{C} 15-\mathrm{C} 16$ & $-81.54(11)$ & $\mathrm{C} 7-\mathrm{C} 5-\mathrm{N} 2-\mathrm{LI}^{\mathrm{i}}$ & $36.11(10)$ \\
\hline $\mathrm{N} 3-\mathrm{C} 8-\mathrm{C} 15-\mathrm{C} 16$ & $91.27(9)$ & $\mathrm{C} 6-\mathrm{C} 5-\mathrm{N} 2-\mathrm{LI}^{\mathrm{i}}$ & $-85.46(9)$ \\
\hline $\mathrm{C} 8-\mathrm{C} 15-\mathrm{C} 16-\mathrm{C} 17$ & $-174.74(7)$ & $\mathrm{C} 7-\mathrm{C} 5-\mathrm{N} 2-\mathrm{LI}$ & $119.48(10)$ \\
\hline $\mathrm{C} 15-\mathrm{C} 16-\mathrm{C} 17-\mathrm{C} 18$ & $172.52(8)$ & $\mathrm{C} 6-\mathrm{C} 5-\mathrm{N} 2-\mathrm{LI}$ & $-2.09(13)$ \\
\hline $\mathrm{O}-\mathrm{C} 19-\mathrm{C} 20-\mathrm{C} 21$ & $-20.85(15)$ & $\mathrm{N} 4-\mathrm{C} 8-\mathrm{N} 3-\mathrm{C} 1$ & $-144.83(9)$ \\
\hline $\mathrm{C} 19-\mathrm{C} 20-\mathrm{C} 21-\mathrm{C} 22$ & $34.32(15)$ & $\mathrm{C} 15-\mathrm{C} 8-\mathrm{N} 3-\mathrm{C} 1$ & $41.78(11)$ \\
\hline $\mathrm{C} 20-\mathrm{C} 21-\mathrm{C} 22-\mathrm{O}$ & $-35.89(14)$ & $\mathrm{N} 4-\mathrm{C} 8-\mathrm{N} 3-\mathrm{C} 9$ & $20.09(13)$ \\
\hline $\mathrm{N} 2-\mathrm{C} 1-\mathrm{N} 1-\mathrm{C} 2$ & $-164.04(8)$ & $\mathrm{C} 15-\mathrm{C} 8-\mathrm{N} 3-\mathrm{C} 9$ & $-153.30(8)$ \\
\hline $\mathrm{N} 3-\mathrm{C} 1-\mathrm{N} 1-\mathrm{C} 2$ & $16.86(13)$ & $\mathrm{N} 1-\mathrm{C} 1-\mathrm{N} 3-\mathrm{C} 8$ & $53.11(12)$ \\
\hline $\mathrm{LI}-\mathrm{C} 1-\mathrm{N} 1-\mathrm{C} 2$ & $172.01(10)$ & $\mathrm{N} 2-\mathrm{C} 1-\mathrm{N} 3-\mathrm{C} 8$ & $-125.98(9)$ \\
\hline $\mathrm{N} 2-\mathrm{C} 1-\mathrm{N} 1-\mathrm{LI}$ & $23.95(9)$ & $\mathrm{LI}-\mathrm{C} 1-\mathrm{N} 3-\mathrm{C} 8$ & $-30.5(2)$ \\
\hline $\mathrm{N} 3-\mathrm{C} 1-\mathrm{N} 1-\mathrm{LI}$ & $-155.15(9)$ & $\mathrm{N} 1-\mathrm{C} 1-\mathrm{N} 3-\mathrm{C} 9$ & $-112.67(9)$ \\
\hline $\mathrm{C} 3-\mathrm{C} 2-\mathrm{N} 1-\mathrm{C} 1$ & $-123.41(9)$ & $\mathrm{N} 2-\mathrm{C} 1-\mathrm{N} 3-\mathrm{C} 9$ & $68.23(10)$ \\
\hline $\mathrm{C} 4-\mathrm{C} 2-\mathrm{N} 1-\mathrm{C} 1$ & $115.74(10)$ & $\mathrm{LI}-\mathrm{C} 1-\mathrm{N} 3-\mathrm{C} 9$ & $163.75(18)$ \\
\hline $\mathrm{C} 3-\mathrm{C} 2-\mathrm{N} 1-\mathrm{LI}$ & $40.51(18)$ & $\mathrm{C} 10-\mathrm{C} 9-\mathrm{N} 3-\mathrm{C} 8$ & $-80.92(10)$ \\
\hline $\mathrm{C} 4-\mathrm{C} 2-\mathrm{N} 1-\mathrm{LI}$ & $-80.34(17)$ & $\mathrm{C} 11-\mathrm{C} 9-\mathrm{N} 3-\mathrm{C} 8$ & $46.94(11)$ \\
\hline $\mathrm{N} 1-\mathrm{C} 1-\mathrm{N} 2-\mathrm{C} 5$ & $-158.57(8)$ & $\mathrm{C} 10-\mathrm{C} 9-\mathrm{N} 3-\mathrm{C} 1$ & $84.59(10)$ \\
\hline $\mathrm{N} 3-\mathrm{C} 1-\mathrm{N} 2-\mathrm{C} 5$ & $20.55(12)$ & $\mathrm{C} 11-\mathrm{C} 9-\mathrm{N} 3-\mathrm{C} 1$ & $-147.55(8)$ \\
\hline $\mathrm{LI}-\mathrm{C} 1-\mathrm{N} 2-\mathrm{C} 5$ & $-136.00(9)$ & $\mathrm{N} 3-\mathrm{C} 8-\mathrm{N} 4-\mathrm{C} 12$ & $-173.36(8)$ \\
\hline $\mathrm{N} 1-\mathrm{C} 1-\mathrm{N} 2-\mathrm{LI}^{\mathrm{i}}$ & $35.43(13)$ & $\mathrm{C} 15-\mathrm{C} 8-\mathrm{N} 4-\mathrm{C} 12$ & $-0.89(14)$ \\
\hline $\mathrm{N} 3-\mathrm{C} 1-\mathrm{N} 2-\mathrm{LI}^{\mathrm{i}}$ & $-145.45(9)$ & $\mathrm{C} 14-\mathrm{C} 12-\mathrm{N} 4-\mathrm{C} 8$ & $120.28(10)$ \\
\hline $\mathrm{LI}-\mathrm{C} 1-\mathrm{N} 2-\mathrm{LI}^{\mathrm{i}}$ & $58.00(11)$ & $\mathrm{C} 13-\mathrm{C} 12-\mathrm{N} 4-\mathrm{C} 8$ & $-119.01(10)$ \\
\hline $\mathrm{N} 1-\mathrm{C} 1-\mathrm{N} 2-\mathrm{LI}$ & $-22.57(9)$ & $\mathrm{C} 21-\mathrm{C} 22-\mathrm{O}-\mathrm{C} 19$ & $23.85(12)$ \\
\hline $\mathrm{N} 3-\mathrm{C} 1-\mathrm{N} 2-\mathrm{LI}$ & $156.55(9)$ & $\mathrm{C} 21-\mathrm{C} 22-\mathrm{O}-\mathrm{LI}$ & $178.16(10)$ \\
\hline $\mathrm{C} 7-\mathrm{C} 5-\mathrm{N} 2-\mathrm{C} 1$ & $-132.84(8)$ & $\mathrm{C} 20-\mathrm{C} 19-\mathrm{O}-\mathrm{C} 22$ & $-1.70(13)$ \\
\hline $\mathrm{C} 6-\mathrm{C} 5-\mathrm{N} 2-\mathrm{C} 1$ & $105.59(9)$ & $\mathrm{C} 20-\mathrm{C} 19-\mathrm{O}-\mathrm{LI}$ & $-157.79(11)$ \\
\hline
\end{tabular}

Symmetry code: (i) $-x+2,-y+1,-z$.

(compound_3) [Bis(tetrahydrofuran)lithium]-di- $\mu$-chlorido- $\left\{\left(N, N^{\prime}\right.\right.$-dicyclohexyl-1-butylamidinato- $\left.\kappa^{2} N^{1}, N^{2}\right)$

$\left[N, N^{\prime}, N^{\prime \prime}, N^{\prime \prime \prime}\right.$-tetracyclohexyl-1-(1-butylamidinato)guanidinato- $\left.\kappa^{2} N^{1}, N^{2}\right]$ holmate(III)\}

Crystal data

$\left[\mathrm{LiHoCl} 2\left(\mathrm{C}_{4} \mathrm{H}_{8} \mathrm{O}\right)_{2}\left(\mathrm{C}_{17} \mathrm{H}_{31} \mathrm{~N}_{2}\right)\left(\mathrm{C}_{30} \mathrm{H}_{53} \mathrm{~N}_{4}\right)\right]$

$M_{r}=1120.17$

Triclinic, $P \overline{1}$

$a=12.909(3) \AA$

$b=15.095(3) \AA$

$$
\begin{aligned}
& c=16.786(3) \AA \\
& \alpha=100.67(3)^{\circ} \\
& \beta=97.20(3)^{\circ} \\
& \gamma=109.50(3)^{\circ} \\
& V=2967.5(12) \AA^{3}
\end{aligned}
$$




\section{$Z=2$}

$F(000)=1184$

$D_{\mathrm{x}}=1.254 \mathrm{Mg} \mathrm{m}^{-3}$

Mo $K \alpha$ radiation, $\lambda=0.71073 \AA$

Cell parameters from 29309 reflections

\section{Data collection}

Stoe IPDS 2T

diffractometer

Radiation source: fine-focus sealed tube

Detector resolution: 6.67 pixels $\mathrm{mm}^{-1}$

area detector scans

Absorption correction: for a sphere

(X-AREA and X-RED; Stoe \& Cie, 2002)

$T_{\min }=0.814, T_{\max }=0.889$

\section{Refinement}

Refinement on $F^{2}$

Least-squares matrix: full

$R\left[F^{2}>2 \sigma\left(F^{2}\right)\right]=0.048$

$w R\left(F^{2}\right)=0.074$

$S=0.91$

12948 reflections

751 parameters

552 restraints

Primary atom site location: heavy-atom method $\theta=2.2-29.5^{\circ}$

$\mu=1.47 \mathrm{~mm}^{-1}$

$T=153 \mathrm{~K}$

Prism, yellow

$0.34 \times 0.20 \times 0.12 \times 0.13$ (radius) $\mathrm{mm}$

29449 measured reflections

12948 independent reflections

9625 reflections with $I>2 \sigma(I)$

$R_{\text {int }}=0.078$

$\theta_{\max }=27.0^{\circ}, \theta_{\min }=2.3^{\circ}$

$h=-16 \rightarrow 16$

$k=-18 \rightarrow 19$

$l=-21 \rightarrow 21$

Secondary atom site location: difference Fourier map

Hydrogen site location: inferred from neighbouring sites

$\mathrm{H}$-atom parameters constrained

$w=1 /\left[\sigma^{2}\left(F_{\mathrm{o}}^{2}\right)+(0.0158 P)^{2}\right]$

where $P=\left(F_{\mathrm{o}}^{2}+2 F_{\mathrm{c}}^{2}\right) / 3$

$(\Delta / \sigma)_{\max }=0.001$

$\Delta \rho_{\max }=0.98$ e $\AA^{-3}$

$\Delta \rho_{\min }=-1.68 \mathrm{e} \AA^{-3}$

Special details

Geometry. All esds (except the esd in the dihedral angle between two 1.s. planes) are estimated using the full covariance matrix. The cell esds are taken into account individually in the estimation of esds in distances, angles and torsion angles; correlations between esds in cell parameters are only used when they are defined by crystal symmetry. An approximate (isotropic) treatment of cell esds is used for estimating esds involving 1.s. planes.

Fractional atomic coordinates and isotropic or equivalent isotropic displacement parameters $\left(\AA^{2}\right)$

\begin{tabular}{llllll}
\hline & $x$ & $y$ & $z$ & $U_{\text {iso }} * U_{\text {eq }}$ & Occ. $(<1)$ \\
\hline HO & $0.88596(2)$ & $0.22098(2)$ & $0.79652(2)$ & $0.02381(5)$ & \\
CL1 & $1.01515(10)$ & $0.18232(10)$ & $0.90859(7)$ & $0.0502(3)$ & \\
CL2 & $0.92641(9)$ & $0.38265(8)$ & $0.90918(6)$ & $0.0426(3)$ & \\
LI & $1.0402(7)$ & $0.3342(8)$ & $0.9944(5)$ & $0.059(2)$ & \\
N1 & $0.7121(3)$ & $0.1321(2)$ & $0.82444(18)$ & $0.0266(7)$ & \\
N2 & $0.7857(3)$ & $0.0572(2)$ & $0.73204(19)$ & $0.0319(8)$ & \\
N3 & $0.6167(3)$ & $-0.0399(2)$ & $0.77010(19)$ & $0.0291(8)$ & \\
N4 & $0.5748(3)$ & $-0.2042(3)$ & $0.7462(2)$ & $0.0400(9)$ & \\
N5 & $0.8511(3)$ & $0.2905(3)$ & $0.68838(19)$ & $0.0295(8)$ & \\
N6 & $1.0136(3)$ & $0.2673(3)$ & $0.7108(2)$ & $0.0315(8)$ & \\
C1 & $0.7056(3)$ & $0.0497(3)$ & $0.7760(2)$ & $0.0277(9)$ & \\
C2 & $0.6417(4)$ & $-0.1197(3)$ & $0.7857(3)$ & $0.0324(10)$ & \\
C3 & $0.7431(3)$ & $-0.0933(3)$ & $0.8544(2)$ & $0.0324(10)$ & \\
H3A & 0.797489 & -0.028883 & 0.854679 & $0.039 *$ & \\
H3B & 0.779768 & -0.140929 & 0.842362 & $0.039 *$ &
\end{tabular}




\begin{tabular}{|c|c|c|c|c|c|}
\hline $\mathrm{C} 4$ & $0.7146(3)$ & -0.0907 (3) & $0.9401(2)$ & $0.0359(10)$ & \\
\hline $\mathrm{H} 4 \mathrm{~A}$ & 0.669097 & -0.049866 & 0.949777 & $0.043 *$ & \\
\hline H4B & 0.669505 & -0.157029 & 0.943057 & $0.043^{*}$ & \\
\hline $\mathrm{C} 5$ & $0.8207(4)$ & -0.0503 & $1.0069(3)$ & $0.0411(11)$ & \\
\hline $\mathrm{H} 5 \mathrm{~A}$ & 0.867870 & 0.013966 & 1.000824 & $0.049 *$ & \\
\hline H5B & 0.863621 & -0.093570 & 0.998476 & $0.049^{*}$ & \\
\hline C6 & 0.7985 & $-0.0397(4)$ & $1.0953(3)$ & $0.0552(14)$ & \\
\hline H6A & 0.870064 & -0.013388 & 1.135146 & $0.083^{*}$ & \\
\hline H6B & 0.757704 & 0.004407 & 1.104798 & $0.083^{*}$ & \\
\hline $\mathrm{H} 6 \mathrm{C}$ & 0.753450 & -0.103239 & 1.102447 & $0.083 *$ & \\
\hline $\mathrm{C} 7$ & $0.6469(4)$ & 0.1335 & $0.8895(2)$ & $0.0309(10)$ & \\
\hline $\mathrm{H} 7$ & 0.589271 & 0.067236 & 0.881307 & $0.037 *$ & \\
\hline $\mathrm{C} 8$ & $0.5874(4)$ & $0.2047(3)$ & $0.8852(3)$ & $0.0376(11)$ & \\
\hline H8A & 0.536844 & 0.185592 & 0.830440 & $0.045^{*}$ & \\
\hline H8B & 0.643569 & 0.270306 & 0.891481 & $0.045^{*}$ & \\
\hline C9 & $0.5193(4)$ & $0.2068(4)$ & $0.9533(3)$ & $0.0500(13)$ & \\
\hline H9A & 0.484465 & 0.255824 & 0.950913 & $0.060^{*}$ & \\
\hline H9B & 0.458330 & 0.142864 & 0.943519 & $0.060 *$ & \\
\hline $\mathrm{C} 10$ & $0.5916(4)$ & $0.2307(4)$ & $1.0384(3)$ & $0.0511(14)$ & \\
\hline H10A & 0.646167 & 0.298083 & 1.051409 & $0.061^{*}$ & \\
\hline H10B & 0.543651 & 0.225935 & 1.080279 & $0.061 *$ & \\
\hline C11 & $0.6542(4)$ & $0.1623(4)$ & $1.0429(3)$ & $0.0490(14)$ & \\
\hline H11A & 0.600010 & 0.096001 & 1.037098 & $0.059^{*}$ & \\
\hline H11B & 0.705383 & 0.182756 & 1.097580 & $0.059^{*}$ & \\
\hline $\mathrm{C} 12$ & 0.7218 & 0.1618 & $0.9748(2)$ & $0.0366(11)$ & \\
\hline $\mathrm{H} 12 \mathrm{~A}$ & 0.779745 & 0.227098 & 0.983208 & $0.044 *$ & \\
\hline H12B & 0.760360 & 0.115525 & 0.977993 & $0.044 *$ & \\
\hline $\mathrm{C} 13$ & 0.7848 & -0.0221 & $0.6659(3)$ & $0.0354(10)$ & \\
\hline H13 & 0.713095 & -0.078757 & 0.656913 & $0.042 *$ & \\
\hline C14 & $0.8830(4)$ & -0.0521 & $0.6889(3)$ & $0.0423(11)$ & \\
\hline H14A & 0.876540 & -0.076142 & 0.739779 & $0.051^{*}$ & \\
\hline H14B & 0.953624 & 0.004912 & 0.700714 & $0.051^{*}$ & \\
\hline $\mathrm{C} 15$ & $0.8872(5)$ & $-0.1321(4)$ & 0.6188 & $0.0543(14)$ & \\
\hline H15A & 0.953655 & -0.148673 & 0.634712 & $0.065^{*}$ & \\
\hline H15B & 0.819270 & -0.191067 & 0.609515 & $0.065^{*}$ & \\
\hline $\mathrm{C} 16$ & $0.8938(5)$ & -0.0969 (4) & $0.5397(3)$ & $0.0566(14)$ & \\
\hline H16A & 0.965698 & -0.041969 & 0.547613 & $0.068^{*}$ & \\
\hline H16B & 0.892221 & -0.149708 & 0.494197 & $0.068^{*}$ & \\
\hline $\mathrm{C} 17$ & $0.7982(5)$ & -0.0651 & $0.5164(3)$ & $0.0573(15)$ & \\
\hline H17A & 0.726760 & -0.121539 & 0.502910 & $0.069^{*}$ & \\
\hline H17B & 0.807019 & -0.039528 & 0.466385 & $0.069^{*}$ & \\
\hline $\mathrm{C} 18$ & $0.7937(4)$ & 0.0131 & $0.5865(3)$ & $0.0442(12)$ & \\
\hline H18A & 0.862233 & 0.071768 & 0.596644 & $0.053^{*}$ & \\
\hline H18B & 0.728130 & 0.030596 & 0.570247 & $0.053^{*}$ & \\
\hline C19A & $0.5097(5)$ & $-0.0672(5)$ & $0.7090(4)$ & $0.0331(14)$ & $0.760(6)$ \\
\hline H19A & 0.507199 & -0.121037 & 0.663003 & $0.040^{*}$ & $0.760(6)$ \\
\hline $\mathrm{C} 20 \mathrm{~A}$ & $0.4073(5)$ & $-0.1061(6)$ & $0.7469(4)$ & $0.0429(18)$ & $0.760(6)$ \\
\hline $\mathrm{H} 20 \mathrm{~A}$ & 0.405727 & -0.054363 & 0.792001 & $0.051^{*}$ & $0.760(6)$ \\
\hline
\end{tabular}




\begin{tabular}{|c|c|c|c|c|c|}
\hline $\mathrm{H} 20 \mathrm{~B}$ & 0.411903 & -0.160300 & 0.770710 & $0.051 *$ & $0.760(6)$ \\
\hline C21A & $0.3004(5)$ & $-0.1414(5)$ & $0.6806(5)$ & 0.0578 (18) & $0.760(6)$ \\
\hline $\mathrm{H} 21 \mathrm{~A}$ & 0.298719 & -0.197599 & 0.638679 & $0.069 *$ & $0.760(6)$ \\
\hline $\mathrm{H} 21 \mathrm{~B}$ & 0.234292 & -0.162916 & 0.706398 & $0.069 *$ & $0.760(6)$ \\
\hline $\mathrm{C} 22 \mathrm{~A}$ & $0.2936(6)$ & -0.0619 (6) & $0.6385(5)$ & 0.0556 (19) & $0.760(6)$ \\
\hline $\mathrm{H} 22 \mathrm{~A}$ & 0.227323 & -0.089060 & 0.592518 & $0.067^{*}$ & $0.760(6)$ \\
\hline $\mathrm{H} 22 \mathrm{~B}$ & 0.283341 & -0.010198 & 0.678791 & $0.067^{*}$ & $0.760(6)$ \\
\hline $\mathrm{C} 23 \mathrm{~A}$ & $0.3971(5)$ & $-0.0187(5)$ & $0.6052(5)$ & $0.0500(18)$ & $0.760(6)$ \\
\hline $\mathrm{H} 23 \mathrm{~A}$ & 0.392214 & 0.037082 & 0.583990 & $0.060^{*}$ & $0.760(6)$ \\
\hline $\mathrm{H} 23 \mathrm{~B}$ & 0.400267 & -0.067578 & 0.558233 & $0.060^{*}$ & $0.760(6)$ \\
\hline $\mathrm{C} 24 \mathrm{~A}$ & $0.5048(5)$ & $0.0152(4)$ & $0.6710(4)$ & $0.0402(14)$ & $0.760(6)$ \\
\hline $\mathrm{H} 24 \mathrm{~A}$ & 0.570868 & 0.037883 & 0.645383 & $0.048^{*}$ & $0.760(6)$ \\
\hline $\mathrm{H} 24 \mathrm{~B}$ & 0.506798 & 0.070018 & 0.714643 & $0.048 *$ & $0.760(6)$ \\
\hline C19B & $0.5113(16)$ & $-0.0319(18)$ & $0.7313(11)$ & $0.042(3)$ & $0.240(6)$ \\
\hline H19B & 0.521468 & 0.038259 & 0.744885 & $0.050 *$ & $0.240(6)$ \\
\hline $\mathrm{C} 20 \mathrm{~B}$ & $0.4137(16)$ & $-0.085(2)$ & $0.7678(14)$ & $0.045(3)$ & $0.240(6)$ \\
\hline $\mathrm{H} 20 \mathrm{C}$ & 0.431333 & -0.060050 & 0.828658 & $0.054^{*}$ & $0.240(6)$ \\
\hline $\mathrm{H} 20 \mathrm{D}$ & 0.401787 & -0.155174 & 0.755793 & $0.054^{*}$ & $0.240(6)$ \\
\hline $\mathrm{C} 21 \mathrm{~B}$ & $0.3073(14)$ & $-0.0722(16)$ & $0.7312(11)$ & $0.050(3)$ & $0.240(6)$ \\
\hline $\mathrm{H} 21 \mathrm{C}$ & 0.243651 & -0.109707 & 0.753477 & $0.060 *$ & $0.240(6)$ \\
\hline $\mathrm{H} 21 \mathrm{D}$ & 0.317008 & -0.003019 & 0.747349 & $0.060 *$ & $0.240(6)$ \\
\hline $\mathrm{C} 22 \mathrm{~B}$ & $0.2814(16)$ & $-0.1062(19)$ & $0.6379(12)$ & $0.053(3)$ & $0.240(6)$ \\
\hline $\mathrm{H} 22 \mathrm{C}$ & 0.266325 & -0.176452 & 0.621643 & $0.064 *$ & $0.240(6)$ \\
\hline $\mathrm{H} 22 \mathrm{D}$ & 0.213338 & -0.095331 & 0.615060 & $0.064^{*}$ & $0.240(6)$ \\
\hline $\mathrm{C} 23 \mathrm{~B}$ & $0.3782(16)$ & $-0.0521(18)$ & $0.6030(14)$ & $0.048(3)$ & $0.240(6)$ \\
\hline $\mathrm{H} 23 \mathrm{C}$ & 0.390539 & 0.017755 & 0.616784 & $0.058 *$ & $0.240(6)$ \\
\hline $\mathrm{H} 23 \mathrm{D}$ & 0.360483 & -0.075539 & 0.542001 & $0.058 *$ & $0.240(6)$ \\
\hline C24B & $0.4863(13)$ & $-0.0667(15)$ & $0.6386(10)$ & $0.041(3)$ & $0.240(6)$ \\
\hline $\mathrm{H} 24 \mathrm{C}$ & 0.475836 & -0.136095 & 0.622681 & $0.049 *$ & $0.240(6)$ \\
\hline $\mathrm{H} 24 \mathrm{D}$ & 0.549806 & -0.029687 & 0.615859 & $0.049^{*}$ & $0.240(6)$ \\
\hline $\mathrm{C} 25$ & $0.5914(4)$ & $-0.2901(3)$ & $0.7618(3)$ & $0.0490(13)$ & \\
\hline $\mathrm{H} 25$ & 0.635051 & -0.275229 & 0.819387 & $0.059 *$ & \\
\hline $\mathrm{C} 26$ & $0.4763(5)$ & -0.3690 (4) & 0.7521 & 0.0653 (16) & \\
\hline $\mathrm{H} 26 \mathrm{~A}$ & 0.486433 & -0.426639 & 0.766690 & $0.078 *$ & \\
\hline $\mathrm{H} 26 \mathrm{~B}$ & 0.434280 & -0.345260 & 0.790956 & $0.078 *$ & \\
\hline $\mathrm{C} 27$ & 0.4085 & $-0.3980(4)$ & 0.6640 & $0.0577(15)$ & \\
\hline $\mathrm{H} 27 \mathrm{~A}$ & 0.335785 & -0.450564 & 0.659609 & $0.069 *$ & \\
\hline $\mathrm{H} 27 \mathrm{~B}$ & 0.392910 & -0.341828 & 0.650828 & $0.069 *$ & \\
\hline $\mathrm{C} 28$ & $0.4717(4)$ & -0.4319 (4) & 0.6028 & $0.0584(15)$ & \\
\hline $\mathrm{H} 28 \mathrm{~A}$ & 0.428556 & -0.446053 & 0.545917 & $0.070 *$ & \\
\hline $\mathrm{H} 28 \mathrm{~B}$ & 0.479290 & -0.492553 & 0.611991 & $0.070 *$ & \\
\hline $\mathrm{C} 29$ & 0.5868 & -0.3566 (4) & $0.6113(3)$ & $0.0555(15)$ & \\
\hline $\mathrm{H} 29 \mathrm{~A}$ & 0.579015 & -0.299086 & 0.594905 & $0.067^{*}$ & \\
\hline $\mathrm{H} 29 \mathrm{~B}$ & 0.627909 & -0.383214 & 0.573360 & $0.067^{*}$ & \\
\hline $\mathrm{C} 30$ & $0.6541(4)$ & -0.3261 (4) & $0.6997(3)$ & $0.0547(13)$ & \\
\hline $\mathrm{H} 30 \mathrm{~A}$ & 0.726971 & -0.274007 & 0.703484 & $0.066^{*}$ & \\
\hline H30B & 0.669539 & -0.381955 & 0.713760 & $0.066^{*}$ & \\
\hline C31 & $0.9532(3)$ & $0.3082(3)$ & $0.6710(2)$ & $0.0312(10)$ & \\
\hline
\end{tabular}




\begin{tabular}{|c|c|c|c|c|}
\hline $\mathrm{C} 32$ & $0.9966(3)$ & $0.3686(3)$ & $0.6105(2)$ & $0.0357(10)$ \\
\hline $\mathrm{H} 32 \mathrm{~A}$ & 1.078453 & 0.404269 & 0.629160 & $0.043^{*}$ \\
\hline H32B & 0.960005 & 0.416616 & 0.609458 & $0.043^{*}$ \\
\hline $\mathrm{C} 33$ & 0.9724 & $0.3042(4)$ & $0.5237(3)$ & $0.0429(12)$ \\
\hline $\mathrm{H} 33 \mathrm{~A}$ & 0.890802 & 0.266289 & 0.506849 & $0.052 *$ \\
\hline H33B & 1.011278 & 0.257846 & 0.525102 & $0.052 *$ \\
\hline $\mathrm{C} 34$ & $1.0095(5)$ & 0.3607 (4) & $0.4587(3)$ & 0.0615 \\
\hline H34A & 0.983018 & 0.315359 & 0.403234 & $0.074 *$ \\
\hline H34B & 0.973580 & 0.409324 & 0.459137 & $0.074^{*}$ \\
\hline $\mathrm{C} 35$ & $1.1355(5)$ & $0.4118(5)$ & $0.4732(4)$ & $0.080(2)$ \\
\hline H35A & 1.154240 & 0.446504 & 0.430002 & $0.120^{*}$ \\
\hline H35B & 1.162139 & 0.457921 & 0.527510 & $0.120^{*}$ \\
\hline $\mathrm{H} 35 \mathrm{C}$ & 1.171581 & 0.363907 & 0.471578 & $0.120^{*}$ \\
\hline $\mathrm{C} 36$ & $0.7672(3)$ & 0.3214 & $0.6481(3)$ & $0.0356(10)$ \\
\hline H36 & 0.805056 & 0.372309 & 0.619663 & $0.043 *$ \\
\hline $\mathrm{C} 37$ & $0.6801(4)$ & 0.2393 & 0.5858 & 0.0585 \\
\hline H37A & 0.646912 & 0.186625 & 0.612946 & $0.070^{*}$ \\
\hline H37B & 0.715939 & 0.214275 & 0.542683 & $0.070^{*}$ \\
\hline $\mathrm{C} 38$ & 0.5862 & $0.2662(5)$ & 0.5443 & $0.0704(18)$ \\
\hline H38A & 0.617167 & 0.313861 & 0.511885 & $0.085^{*}$ \\
\hline H38B & 0.528600 & 0.207747 & 0.505750 & $0.085^{*}$ \\
\hline C39 & $0.5330(4)$ & 0.3089 (4) & $0.6087(3)$ & 0.0543 \\
\hline H39A & 0.476913 & 0.331023 & 0.581531 & $0.065^{*}$ \\
\hline Н39В & 0.493639 & 0.258428 & 0.636125 & $0.065^{*}$ \\
\hline $\mathrm{C} 40$ & $0.6187(4)$ & 0.3914 & 0.6715 (3) & $0.0616(16)$ \\
\hline $\mathrm{H} 40 \mathrm{~A}$ & 0.582190 & 0.415206 & 0.714736 & $0.074 *$ \\
\hline $\mathrm{H} 40 \mathrm{~B}$ & 0.651283 & 0.444841 & 0.644881 & $0.074 *$ \\
\hline $\mathrm{C} 41$ & 0.7133 & $0.3643(4)$ & 0.7123 & 0.0550 \\
\hline H41A & 0.770903 & 0.422789 & 0.750790 & $0.066^{*}$ \\
\hline H41B & 0.682580 & 0.316731 & 0.744805 & $0.066^{*}$ \\
\hline $\mathrm{C} 42$ & $1.1312(3)$ & $0.2840(4)$ & $0.7071(3)$ & $0.0308(10)$ \\
\hline $\mathrm{H} 42$ & 1.145267 & 0.300954 & 0.653577 & $0.037^{*}$ \\
\hline $\mathrm{C} 43$ & $1.1522(3)$ & $0.1916(3)$ & 0.7092 & 0.0365 \\
\hline $\mathrm{H} 43 \mathrm{~A}$ & 1.105112 & 0.140195 & 0.659777 & $0.044 *$ \\
\hline H43B & 1.130249 & 0.169962 & 0.758879 & $0.044^{*}$ \\
\hline $\mathrm{C} 44$ & $1.2756(4)$ & $0.2061(4)$ & $0.7110(3)$ & 0.0479 (13) \\
\hline $\mathrm{H} 44 \mathrm{~A}$ & 1.287276 & 0.145537 & 0.715685 & $0.057^{*}$ \\
\hline H44B & 1.295429 & 0.220700 & 0.658566 & $0.057 *$ \\
\hline $\mathrm{C} 45$ & $1.3510(3)$ & $0.2875(4)$ & $0.7826(3)$ & $0.0464(12)$ \\
\hline $\mathrm{H} 45 \mathrm{~A}$ & 1.430363 & 0.297481 & 0.780586 & $0.056^{*}$ \\
\hline H45B & 1.336410 & 0.270185 & 0.835252 & $0.056^{*}$ \\
\hline C46 & $1.3312(3)$ & $0.3804(4)$ & $0.7796(3)$ & $0.0473(12)$ \\
\hline H46A & 1.353685 & 0.401361 & 0.729874 & $0.057^{*}$ \\
\hline H46B & 1.378416 & 0.431883 & 0.828959 & $0.057^{*}$ \\
\hline $\mathrm{C} 47$ & 1.2085 & 0.3668 & 0.7774 & $0.0376(10)$ \\
\hline H47A & 1.188910 & 0.354205 & 0.830431 & $0.045^{*}$ \\
\hline H47B & 1.197492 & 0.427205 & 0.771297 & $0.045^{*}$ \\
\hline O1A & $1.2012(6)$ & $0.3939(5)$ & $1.0059(6)$ & $0.068(2)$ \\
\hline
\end{tabular}




\begin{tabular}{|c|c|c|c|c|c|}
\hline $\mathrm{C} 48 \mathrm{~A}$ & $1.2625(9)$ & $0.4994(8)$ & $1.0228(10)$ & 0.080 & $0.663(11)$ \\
\hline H48A & 1.274964 & 0.519623 & 0.970858 & $0.096^{*}$ & $0.663(11)$ \\
\hline H48B & 1.220589 & 0.535503 & 1.051151 & $0.096^{*}$ & $0.663(11)$ \\
\hline C49A & $1.3690(8)$ & $0.5161(8)$ & $1.0764(8)$ & $0.090(3)$ & $0.663(11)$ \\
\hline $\mathrm{H} 49 \mathrm{~A}$ & 1.431192 & 0.567465 & 1.063643 & $0.107^{*}$ & $0.663(11)$ \\
\hline H49B & 1.365638 & 0.535823 & 1.135336 & $0.107^{*}$ & 0.663 \\
\hline $\mathrm{C} 50 \mathrm{~A}$ & $1.3853(8)$ & $0.4236(8)$ & $1.0590(9)$ & $0.091(3)$ & $0.663(11)$ \\
\hline H50A & 1.436979 & 0.424596 & 1.020053 & $0.109^{*}$ & $0.663(11)$ \\
\hline H50B & 1.418617 & 0.412419 & 1.110831 & $0.109^{*}$ & $0.663(11)$ \\
\hline C51A & $1.2766(6)$ & $0.3465(6)$ & $1.0228(7)$ & $0.068(2)$ & 0.663 \\
\hline H51A & 1.280535 & 0.304691 & 0.971227 & $0.081^{*}$ & 0.663 \\
\hline H51B & 1.252386 & 0.305921 & 1.062064 & $0.081^{*}$ & 0.663 \\
\hline O1B & $1.1907(11)$ & $0.4215(12)$ & $1.0355(11)$ & $0.071(3)$ & 0.337 \\
\hline C48B & $1.2503(16)$ & $0.5020(18)$ & $1.0006(18)$ & $0.077(3)$ & 0.337 \\
\hline $\mathrm{H} 48 \mathrm{C}$ & 1.235041 & 0.560929 & 1.022480 & $0.093^{*}$ & $0.337(11)$ \\
\hline H48D & 1.229113 & 0.484365 & 0.939389 & $0.093^{*}$ & $0.337(11)$ \\
\hline C49B & $1.3702(14)$ & $0.5162(14)$ & $1.0291(17)$ & 0.085 & $0.337(11)$ \\
\hline $\mathrm{H} 49 \mathrm{C}$ & 1.400914 & 0.560276 & 1.085066 & $0.102^{*}$ & $0.337(11)$ \\
\hline H49D & 1.416696 & 0.542913 & 0.990343 & $0.102 *$ & $0.337(11)$ \\
\hline $\mathrm{C} 50 \mathrm{~B}$ & $1.3656(16)$ & $0.4157(16)$ & $1.0301(14)$ & $0.082(3)$ & $0.337(11)$ \\
\hline $\mathrm{H} 50 \mathrm{C}$ & 1.352174 & 0.375145 & 0.973496 & $0.098^{*}$ & $0.337(11)$ \\
\hline H50D & 1.434669 & 0.416376 & 1.063665 & $0.098^{*}$ & $0.337(11)$ \\
\hline C51B & $1.2669(13)$ & $0.3833(16)$ & $1.0698(14)$ & 0.078 & 0.337 \\
\hline $\mathrm{H} 51 \mathrm{C}$ & 1.233379 & 0.311731 & 1.057091 & $0.093^{*}$ & $0.337(11)$ \\
\hline H51D & 1.288978 & 0.408865 & 1.130623 & $0.093^{*}$ & 0.337 \\
\hline $\mathrm{O} 2 \mathrm{~A}$ & $1.0007(16)$ & $0.3190(12)$ & $1.0994(5)$ & $0.0804(18)$ & 0.823 \\
\hline C52A & $0.9608(13)$ & $0.3830(10)$ & $1.1520(5)$ & $0.086(2)$ & 0.823 \\
\hline H52A & 0.883103 & 0.374213 & 1.127587 & $0.103 *$ & 0.823 \\
\hline H52B & 1.009542 & 0.451516 & 1.159370 & $0.103 *$ & 0.823 \\
\hline $\mathrm{C} 53 \mathrm{~A}$ & $0.9650(10)$ & $0.3558(8)$ & $1.2315(5)$ & $0.101(3)$ & 0.823 \\
\hline H53A & 1.022040 & 0.409209 & 1.275267 & $0.121^{*}$ & 0.823 \\
\hline H53B & 0.891206 & 0.341805 & 1.247827 & $0.121^{*}$ & 0.823 \\
\hline C54A & $0.9944(9)$ & $0.2699(7)$ & $1.2204(4)$ & $0.092(2)$ & 0.823 \\
\hline H54A & 1.066163 & 0.283501 & 1.258211 & $0.110^{*}$ & 0.823 \\
\hline H54B & 0.935406 & 0.216153 & 1.233507 & $0.110^{*}$ & 0.823 \\
\hline C55A & $1.0048(9)$ & $0.2429(7)$ & $1.1357(5)$ & $0.090(2)$ & 0.823 \\
\hline H55A & 1.076793 & 0.233467 & 1.133376 & $0.108^{*}$ & 0.823 \\
\hline H55B & 0.942657 & 0.181679 & 1.105878 & $0.108^{*}$ & 0.823 \\
\hline $\mathrm{O} 2 \mathrm{~B}$ & $1.001(8)$ & $0.329(6)$ & $1.0966(19)$ & $0.084(3)$ & $0.177(11)$ \\
\hline $\mathrm{C} 52 \mathrm{~B}$ & $1.054(4)$ & $0.291(3)$ & $1.158(2)$ & $0.088(4)$ & 0.177 \\
\hline $\mathrm{H} 52 \mathrm{C}$ & 1.130016 & 0.294988 & 1.149183 & $0.106^{*}$ & $0.177(11)$ \\
\hline H52D & 1.008073 & 0.222688 & 1.154448 & $0.106^{*}$ & 0.177 \\
\hline C53B & $1.060(4)$ & $0.355(3)$ & $1.2372(17)$ & $0.093(4)$ & 0.177 \\
\hline $\mathrm{H} 53 \mathrm{C}$ & 1.042864 & 0.316691 & 1.279167 & $0.112^{*}$ & 0.177 \\
\hline H53D & 1.136071 & 0.404992 & 1.257247 & $0.112^{*}$ & $0.177(11)$ \\
\hline C54B & $0.978(4)$ & $0.400(3)$ & $1.224(2)$ & $0.095(4)$ & 0.177 (11) \\
\hline $\mathrm{H} 54 \mathrm{C}$ & 1.011332 & 0.470084 & 1.249695 & $0.114^{*}$ & 0.177 (11) \\
\hline H54D & 0.912345 & 0.370614 & 1.248044 & $0.114 *$ & 0.177 \\
\hline
\end{tabular}




\begin{tabular}{llllll} 
C55B & $0.943(6)$ & $0.383(5)$ & $1.134(2)$ & $0.088(4)$ & $0.177(11)$ \\
H55C & 0.860697 & 0.347129 & 1.116540 & $0.106^{*}$ & $0.177(11)$ \\
H55D & 0.961018 & 0.445725 & 1.117958 & $0.106^{*}$ & $0.177(11)$ \\
\hline
\end{tabular}

Atomic displacement parameters $\left(\AA^{2}\right)$

\begin{tabular}{|c|c|c|c|c|c|c|}
\hline & $U^{11}$ & $U^{22}$ & $U^{33}$ & $U^{12}$ & $U^{13}$ & $U^{23}$ \\
\hline $\mathrm{HO}$ & $0.02778(9)$ & $0.02410(10)$ & $0.02243(8)$ & $0.01058(7)$ & $0.00729(6)$ & $0.00895(7)$ \\
\hline CL1 & $0.0550(7)$ & $0.0666(9)$ & $0.0433(6)$ & $0.0348(7)$ & $0.0066(5)$ & $0.0259(6)$ \\
\hline CL2 & $0.0553(7)$ & $0.0321(7)$ & $0.0357(6)$ & $0.0152(6)$ & $0.0048(5)$ & $0.0012(5)$ \\
\hline LI & $0.057(5)$ & $0.074(7)$ & $0.040(5)$ & $0.016(5)$ & $0.000(4)$ & $0.018(5)$ \\
\hline N1 & $0.0327(18)$ & 0.0240 (19) & $0.0251(17)$ & $0.0121(15)$ & $0.0102(14)$ & $0.0051(15)$ \\
\hline N2 & $0.044(2)$ & $0.025(2)$ & $0.0302(18)$ & $0.0167(17)$ & $0.0132(15)$ & $0.0046(16)$ \\
\hline N3 & $0.0317(18)$ & 0.0219 (19) & $0.0303(18)$ & $0.0061(16)$ & $0.0033(14)$ & $0.0073(16)$ \\
\hline N4 & $0.053(2)$ & $0.027(2)$ & $0.034(2)$ & 0.0082 (19) & $0.0046(17)$ & $0.0087(18)$ \\
\hline N5 & $0.0266(18)$ & $0.039(2)$ & $0.0319(18)$ & $0.0179(16)$ & $0.0086(14)$ & $0.0160(17)$ \\
\hline N6 & 0.0255 (17) & $0.043(2)$ & 0.0348 (19) & $0.0171(17)$ & $0.0101(14)$ & $0.0201(18)$ \\
\hline $\mathrm{C} 1$ & $0.034(2)$ & $0.025(2)$ & $0.026(2)$ & $0.0114(19)$ & 0.0075 & $0.0094(18)$ \\
\hline $\mathrm{C} 2$ & $0.040(2)$ & $0.026(3)$ & $0.031(2)$ & $0.010(2)$ & 0.0107 (18) & $0.008(2)$ \\
\hline $\mathrm{C} 3$ & $0.040(2)$ & 0.029 (2) & $0.031(2)$ & $0.014(2)$ & $0.0075(18)$ & 0.0108 (19) \\
\hline $\mathrm{C} 4$ & $0.040(2)$ & $0.034(3)$ & $0.034(2)$ & $0.010(2)$ & $0.0089(18)$ & $0.015(2)$ \\
\hline $\mathrm{C} 5$ & $0.049(3)$ & $0.042(3)$ & $0.036(2)$ & $0.018(2)$ & $0.006(2)$ & $0.015(2)$ \\
\hline C6 & $0.065(3)$ & $0.071(4)$ & $0.030(2)$ & $0.029(3)$ & $0.002(2)$ & $0.010(3)$ \\
\hline $\mathrm{C} 7$ & $0.039(2)$ & $0.028(3)$ & $0.029(2)$ & $0.012(2)$ & $0.0122(18)$ & $0.008(2)$ \\
\hline $\mathrm{C} 8$ & $0.040(3)$ & $0.040(3)$ & $0.034(2)$ & $0.018(2)$ & 0.0116 (19) & $0.004(2)$ \\
\hline C9 & $0.049(3)$ & $0.065(4)$ & $0.044(3)$ & $0.031(3)$ & $0.019(2)$ & $0.007(3)$ \\
\hline C10 & $0.051(3)$ & $0.062(4)$ & $0.037(3)$ & $0.021(3)$ & $0.021(2)$ & $-0.002(3)$ \\
\hline $\mathrm{C} 11$ & $0.053(3)$ & $0.063(4)$ & $0.026(2)$ & $0.016(3)$ & $0.015(2)$ & $0.006(2)$ \\
\hline $\mathrm{C} 12$ & $0.040(2)$ & $0.048(3)$ & $0.026(2)$ & $0.022(2)$ & $0.0086(18)$ & 0.009 (2) \\
\hline $\mathrm{C} 13$ & $0.045(3)$ & $0.028(3)$ & $0.037(2)$ & $0.017(2)$ & 0.0156 (19) & 0.007 (2) \\
\hline $\mathrm{C} 14$ & $0.059(3)$ & $0.045(3)$ & $0.035(2)$ & $0.029(3)$ & $0.021(2)$ & $0.015(2)$ \\
\hline $\mathrm{C} 15$ & $0.083(4)$ & $0.052(3)$ & $0.050(3)$ & $0.046(3)$ & $0.029(3)$ & $0.014(3)$ \\
\hline $\mathrm{C} 16$ & $0.087(4)$ & $0.058(4)$ & $0.044(3)$ & $0.042(3)$ & $0.034(3)$ & $0.015(3)$ \\
\hline $\mathrm{C} 17$ & $0.087(4)$ & $0.067(4)$ & $0.033(3)$ & $0.048(3)$ & $0.020(2)$ & 0.007 (3) \\
\hline $\mathrm{C} 18$ & $0.068(3)$ & $0.044(3)$ & $0.034(2)$ & $0.036(3)$ & $0.016(2)$ & $0.010(2)$ \\
\hline C19A & $0.035(3)$ & $0.028(3)$ & $0.034(3)$ & $0.010(3)$ & $-0.002(2)$ & $0.010(2)$ \\
\hline $\mathrm{C} 20 \mathrm{~A}$ & $0.038(3)$ & $0.041(4)$ & $0.044(3)$ & $0.008(3)$ & 0.005 & $0.011(3)$ \\
\hline $\mathrm{C} 21 \mathrm{~A}$ & $0.041(3)$ & $0.052(4)$ & $0.067(4)$ & $0.008(3)$ & $-0.002(3)$ & $0.011(3)$ \\
\hline $\mathrm{C} 22 \mathrm{~A}$ & $0.047(3)$ & $0.040(4)$ & $0.068(3)$ & $0.015(3)$ & -0.015 & $0.006(3)$ \\
\hline $\mathrm{C} 23 \mathrm{~A}$ & $0.051(3)$ & $0.034(4)$ & $0.052(3)$ & 0.009 (3) & $-0.013(3)$ & $0.008(3)$ \\
\hline $\mathrm{C} 24 \mathrm{~A}$ & $0.044(3)$ & $0.031(3)$ & $0.041(3)$ & $0.013(2)$ & $-0.006(2)$ & $0.008(2)$ \\
\hline C19B & $0.039(4)$ & $0.036(5)$ & $0.042(5)$ & $0.006(5)$ & $0.003(4)$ & $0.009(5)$ \\
\hline $\mathrm{C} 20 \mathrm{~B}$ & $0.038(4)$ & $0.041(5)$ & $0.047(5)$ & 0.007 (4) & $0.000(4)$ & $0.009(5)$ \\
\hline $\mathrm{C} 21 \mathrm{~B}$ & $0.038(4)$ & $0.045(5)$ & $0.056(5)$ & 0.009 (4) & $-0.001(4)$ & $0.008(5)$ \\
\hline $\mathrm{C} 22 \mathrm{~B}$ & $0.043(5)$ & $0.042(5)$ & $0.062(5)$ & $0.010(5)$ & $-0.012(4)$ & $0.011(5)$ \\
\hline $\mathrm{C} 23 \mathrm{~B}$ & $0.048(5)$ & $0.036(5)$ & $0.051(5)$ & $0.011(5)$ & $-0.012(4)$ & $0.011(5)$ \\
\hline C24B & $0.043(4)$ & $0.031(5)$ & $0.041(4)$ & $0.006(4)$ & $-0.002(4)$ & $0.010(4)$ \\
\hline $\mathrm{C} 25$ & $0.073(3)$ & $0.026(3)$ & $0.038(3)$ & $0.011(3)$ & $-0.001(2)$ & 0.009 (2) \\
\hline
\end{tabular}




\begin{tabular}{|c|c|c|c|c|c|c|}
\hline $\mathrm{C} 26$ & $0.096(4)$ & 0.029 & $0.051(3)$ & -0.001 & $0.022(3)$ & $0.006(3)$ \\
\hline $\mathrm{C} 27$ & $0.062(3)$ & $0.033(3)$ & $0.060(3)$ & $0.001(3)$ & 0.009 (3) & -0.002 \\
\hline $\mathrm{C} 28$ & 0.080 & 0.037 (3) & 0.048 & $0.023(3)$ & -0.006 & -0.005 \\
\hline $\mathrm{C} 29$ & $0.073(4)$ & $0.051(4)$ & 0.047 (3) & $0.031(3)$ & 0.015 & $0.004(3)$ \\
\hline $\mathrm{C} 30$ & $0.069(3)$ & 0.032 & 0.060 & $0.019(3)$ & 0.005 & $0.010(3)$ \\
\hline $\mathrm{C} 31$ & $0.033(2)$ & 0.038 & $0.026(2)$ & $0.015(2)$ & $0.0110(17)$ & $0.010(2)$ \\
\hline $\mathrm{C} 32$ & $0.039(2)$ & 0.038 & $0.035(2)$ & $0.015(2)$ & $0.0110(18)$ & $0.015(2)$ \\
\hline $\mathrm{C} 33$ & 0.050 & 0.047 (3) & $0.033(2)$ & $0.015(2)$ & $0.017(2)$ & $0.011(2)$ \\
\hline C34 & $0.083(4)$ & $0.071(4)$ & $0.038(3)$ & $0.027(3)$ & $0.025(3)$ & $0.023(3)$ \\
\hline $\mathrm{C} 35$ & $0.084(4)$ & $0.095(5)$ & 0.065 (4) & $0.023(4)$ & $0.041(3)$ & $0.032(4)$ \\
\hline $\mathrm{C} 36$ & $0.032(2)$ & $0.048(3)$ & $0.037(2)$ & 0.019 (2) & $0.0106(18)$ & $0.024(2)$ \\
\hline $\mathrm{C} 37$ & $0.050(3)$ & $0.068(4)$ & $0.059(3)$ & $0.036(3)$ & $0.002(2)$ & $-0.001(3)$ \\
\hline $\mathrm{C} 38$ & 0.049 (3) & $0.096(5)$ & $0.064(4)$ & $0.042(3)$ & $-0.011(3)$ & $0.001(3)$ \\
\hline C39 & $0.037(3)$ & $0.070(4)$ & $0.064(3)$ & $0.028(3)$ & $0.008(2)$ & $0.022(3)$ \\
\hline $\mathrm{C} 40$ & $0.062(3)$ & $0.065(4)$ & $0.071(4)$ & $0.046(3)$ & 0.008 & 0.009 (3) \\
\hline $\mathrm{C} 41$ & $0.059(3)$ & $0.061(4)$ & $0.049(3)$ & $0.037(3)$ & $0.002(2)$ & $0.001(3)$ \\
\hline $\mathrm{C} 42$ & $0.027(3)$ & 0.039 & $0.030(2)$ & $0.013(2)$ & $0.0072(18)$ & $0.0128(19$ \\
\hline C43 & $0.032(2)$ & 0.032 & $0.044(3)$ & $0.011(2)$ & 0.0085 (19) & $0.007(2)$ \\
\hline C44 & $0.041(3)$ & 0.045 & 0.060 & $0.021(2)$ & 0.014 & 0.006 \\
\hline $\mathrm{C} 45$ & $0.028(2)$ & $0.056(3)$ & $0.051(3)$ & $0.011(2)$ & $0.003(2)$ & $0.013(3)$ \\
\hline $\mathrm{C} 46$ & $0.033(2)$ & $0.044(3)$ & $0.054(3)$ & $0.004(2)$ & $0.001(2)$ & $0.010(3)$ \\
\hline $\mathrm{C} 47$ & $0.037(2)$ & $0.030(3)$ & $0.044(3)$ & $0.010(2)$ & 0.0058 (19) & $0.009(2)$ \\
\hline O1A & $0.048(3)$ & 0.049 & $0.092(5)$ & 0.017 (3) & -0.009 & -0.003 \\
\hline C48A & $0.062(4)$ & $0.057(4)$ & $0.103(6)$ & $0.021(3)$ & $-0.007(4)$ & -0.005 (4) \\
\hline C49A & 0.065 (4) & $0.073(4)$ & $0.098(6)$ & $0.010(3)$ & -0.014 & $-0.007(5)$ \\
\hline $\mathrm{C} 50 \mathrm{~A}$ & $0.066(4)$ & $0.072(4)$ & $0.108(6)$ & $0.010(4)$ & $-0.026(4)$ & $0.018(5)$ \\
\hline C51A & 0.054 (4) & 0.057 (4) & $0.087(5)$ & $0.026(3)$ & $-0.007(4)$ & 0.009 (4) \\
\hline O1B & $0.052(4)$ & $0.058(5)$ & $0.092(6)$ & $0.017(4)$ & -0.005 & $0.009(5)$ \\
\hline C48B & $0.064(5)$ & $0.058(5)$ & $0.092(6)$ & $0.016(4)$ & $-0.006(5)$ & $0.001(5)$ \\
\hline C49B & $0.064(5)$ & $0.067(5)$ & $0.102(7)$ & $0.016(4)$ & $-0.012(5)$ & $0.006(5)$ \\
\hline C50B & $0.062(5)$ & $0.069(5)$ & $0.095(7)$ & 0.019 (4) & $-0.018(5)$ & $0.008(5)$ \\
\hline C51B & 0.061 (4) & $0.061(5)$ & $0.095(6)$ & $0.017(4)$ & $-0.012(4)$ & $0.010(5)$ \\
\hline $\mathrm{O} 2 \mathrm{~A}$ & $0.135(3)$ & $0.077(5)$ & $0.038(2)$ & $0.044(3)$ & $0.019(2)$ & $0.026(2)$ \\
\hline $\mathrm{C} 52 \mathrm{~A}$ & $0.150(5)$ & $0.071(4)$ & $0.048(4)$ & $0.046(4)$ & $0.026(4)$ & $0.027(4)$ \\
\hline C53A & $0.167(5)$ & $0.087(5)$ & 0.055 (4) & $0.045(5)$ & $0.030(4)$ & $0.028(4)$ \\
\hline C54A & $0.148(5)$ & $0.091(5)$ & 0.054 & $0.050(4)$ & $0.031(4)$ & $0.037(4)$ \\
\hline C55A & $0.145(5)$ & $0.083(5)$ & 0.053 (4) & $0.047(4)$ & 0.009 (4) & $0.034(4)$ \\
\hline $\mathrm{O} 2 \mathrm{~B}$ & $0.141(5)$ & $0.077(6)$ & $0.045(5)$ & $0.048(5)$ & $0.019(5)$ & $0.029(5)$ \\
\hline C52B & $0.144(6)$ & $0.082(6)$ & $0.050(5)$ & $0.049(5)$ & $0.021(5)$ & $0.030(5)$ \\
\hline C53B & $0.152(6)$ & $0.085(6)$ & $0.052(5)$ & $0.046(6)$ & $0.025(5)$ & $0.030(5)$ \\
\hline C54B & $0.158(6)$ & $0.080(6)$ & $0.053(6)$ & $0.043(6)$ & $0.025(6)$ & $0.029(6)$ \\
\hline C55B & $0.149(6)$ & $0.077(6)$ & 0.048 & $0.046(5)$ & 0.025 & $0.026(5)$ \\
\hline
\end{tabular}

Geometric parameters $\left(\AA,{ }^{\circ}\right)$

\begin{tabular}{llll}
\hline $\mathrm{HO}-\mathrm{N} 5$ & $2.327(3)$ & $\mathrm{C} 26-\mathrm{C} 27$ & $1.525(7)$ \\
$\mathrm{HO}-\mathrm{N} 6$ & $2.339(3)$ & $\mathrm{C} 26-\mathrm{H} 26 \mathrm{~A}$ & 0.9900 \\
$\mathrm{HO}-\mathrm{N} 2$ & $2.341(4)$ & $\mathrm{C} 26-\mathrm{H} 26 \mathrm{~B}$ & 0.9900
\end{tabular}




\begin{tabular}{|c|c|c|c|}
\hline $\mathrm{HO}-\mathrm{N} 1$ & $2.354(3)$ & $\mathrm{C} 27-\mathrm{C} 28$ & $1.509(7)$ \\
\hline $\mathrm{HO}-\mathrm{CL} 1$ & $2.6326(13)$ & $\mathrm{C} 27-\mathrm{H} 27 \mathrm{~A}$ & 0.9900 \\
\hline $\mathrm{HO}-\mathrm{CL} 2$ & $2.6453(15)$ & $\mathrm{C} 27-\mathrm{H} 27 \mathrm{~B}$ & 0.9900 \\
\hline $\mathrm{HO}-\mathrm{C} 31$ & $2.766(4)$ & $\mathrm{C} 28-\mathrm{C} 29$ & $1.510(7)$ \\
\hline $\mathrm{HO}-\mathrm{C} 1$ & $2.770(4)$ & $\mathrm{C} 28-\mathrm{H} 28 \mathrm{~A}$ & 0.9900 \\
\hline $\mathrm{HO}-\mathrm{LI}$ & $3.447(8)$ & $\mathrm{C} 28-\mathrm{H} 28 \mathrm{~B}$ & 0.9900 \\
\hline CL1-LI & $2.366(10)$ & $\mathrm{C} 29-\mathrm{C} 30$ & $1.524(7)$ \\
\hline CL2-LI & $2.306(9)$ & $\mathrm{C} 29-\mathrm{H} 29 \mathrm{~A}$ & 0.9900 \\
\hline $\mathrm{LI}-\mathrm{O} 2 \mathrm{~B}$ & $1.86(5)$ & $\mathrm{C} 29-\mathrm{H} 29 \mathrm{~B}$ & 0.9900 \\
\hline $\mathrm{LI}-\mathrm{O} 1 \mathrm{~B}$ & $1.898(17)$ & $\mathrm{C} 30-\mathrm{H} 30 \mathrm{~A}$ & 0.9900 \\
\hline $\mathrm{LI}-\mathrm{O} 2 \mathrm{~A}$ & $1.928(13)$ & $\mathrm{C} 30-\mathrm{H} 30 \mathrm{~B}$ & 0.9900 \\
\hline $\mathrm{LI}-\mathrm{O} 1 \mathrm{~A}$ & $1.937(11)$ & $\mathrm{C} 31-\mathrm{C} 32$ & $1.523(6)$ \\
\hline $\mathrm{N} 1-\mathrm{C} 1$ & $1.324(5)$ & $\mathrm{C} 32-\mathrm{C} 33$ & $1.524(6)$ \\
\hline $\mathrm{N} 1-\mathrm{C} 7$ & $1.461(5)$ & $\mathrm{C} 32-\mathrm{H} 32 \mathrm{~A}$ & 0.9900 \\
\hline $\mathrm{N} 2-\mathrm{C} 1$ & $1.331(5)$ & C $32-\mathrm{H} 32 \mathrm{~B}$ & 0.9900 \\
\hline $\mathrm{N} 2-\mathrm{C} 13$ & $1.469(5)$ & $\mathrm{C} 33-\mathrm{C} 34$ & $1.533(6)$ \\
\hline $\mathrm{N} 3-\mathrm{C} 2$ & $1.407(5)$ & $\mathrm{C} 33-\mathrm{H} 33 \mathrm{~A}$ & 0.9900 \\
\hline $\mathrm{N} 3-\mathrm{C} 1$ & $1.429(5)$ & C $33-\mathrm{H} 33 \mathrm{~B}$ & 0.9900 \\
\hline N3- $\mathrm{C} 19 \mathrm{~B}$ & $1.49(2)$ & $\mathrm{C} 34-\mathrm{C} 35$ & $1.514(7)$ \\
\hline N3-C19A & $1.496(6)$ & $\mathrm{C} 34-\mathrm{H} 34 \mathrm{~A}$ & 0.9900 \\
\hline $\mathrm{N} 4-\mathrm{C} 2$ & $1.272(5)$ & $\mathrm{C} 34-\mathrm{H} 34 \mathrm{~B}$ & 0.9900 \\
\hline $\mathrm{N} 4-\mathrm{C} 25$ & $1.447(6)$ & $\mathrm{C} 35-\mathrm{H} 35 \mathrm{~A}$ & 0.9800 \\
\hline N5-C31 & $1.336(5)$ & $\mathrm{C} 35-\mathrm{H} 35 \mathrm{~B}$ & 0.9800 \\
\hline N5-C36 & $1.456(5)$ & $\mathrm{C} 35-\mathrm{H} 35 \mathrm{C}$ & 0.9800 \\
\hline N6-C31 & $1.334(5)$ & $\mathrm{C} 36-\mathrm{C} 37$ & $1.479(6)$ \\
\hline $\mathrm{N} 6-\mathrm{C} 42$ & $1.465(5)$ & $\mathrm{C} 36-\mathrm{C} 41$ & $1.515(6)$ \\
\hline $\mathrm{C} 2-\mathrm{C} 3$ & $1.518(6)$ & $\mathrm{C} 36-\mathrm{H} 36$ & 1.0000 \\
\hline $\mathrm{C} 3-\mathrm{C} 4$ & $1.527(5)$ & $\mathrm{C} 37-\mathrm{C} 38$ & $1.530(6)$ \\
\hline $\mathrm{C} 3-\mathrm{H} 3 \mathrm{~A}$ & 0.9900 & $\mathrm{C} 37-\mathrm{H} 37 \mathrm{~A}$ & 0.9900 \\
\hline $\mathrm{C} 3-\mathrm{H} 3 \mathrm{~B}$ & 0.9900 & C37-H37B & 0.9900 \\
\hline $\mathrm{C} 4-\mathrm{C} 5$ & $1.519(6)$ & $\mathrm{C} 38-\mathrm{C} 39$ & $1.509(7)$ \\
\hline $\mathrm{C} 4-\mathrm{H} 4 \mathrm{~A}$ & 0.9900 & $\mathrm{C} 38-\mathrm{H} 38 \mathrm{~A}$ & 0.9900 \\
\hline $\mathrm{C} 4-\mathrm{H} 4 \mathrm{~B}$ & 0.9900 & $\mathrm{C} 38-\mathrm{H} 38 \mathrm{~B}$ & 0.9900 \\
\hline $\mathrm{C} 5-\mathrm{C} 6$ & $1.536(6)$ & $\mathrm{C} 39-\mathrm{C} 40$ & $1.479(7)$ \\
\hline $\mathrm{C} 5-\mathrm{H} 5 \mathrm{~A}$ & 0.9900 & $\mathrm{C} 39-\mathrm{H} 39 \mathrm{~A}$ & 0.9900 \\
\hline $\mathrm{C} 5-\mathrm{H} 5 \mathrm{~B}$ & 0.9900 & C39-H39B & 0.9900 \\
\hline C6-H6A & 0.9800 & $\mathrm{C} 40-\mathrm{C} 41$ & $1.536(6)$ \\
\hline C6-H6B & 0.9800 & $\mathrm{C} 40-\mathrm{H} 40 \mathrm{~A}$ & 0.9900 \\
\hline C6-H6C & 0.9800 & $\mathrm{C} 40-\mathrm{H} 40 \mathrm{~B}$ & 0.9900 \\
\hline $\mathrm{C} 7-\mathrm{C} 12$ & $1.522(6)$ & $\mathrm{C} 41-\mathrm{H} 41 \mathrm{~A}$ & 0.9900 \\
\hline $\mathrm{C} 7-\mathrm{C} 8$ & $1.522(6)$ & $\mathrm{C} 41-\mathrm{H} 41 \mathrm{~B}$ & 0.9900 \\
\hline $\mathrm{C} 7-\mathrm{H} 7$ & 1.0000 & $\mathrm{C} 42-\mathrm{C} 47$ & $1.509(6)$ \\
\hline $\mathrm{C} 8-\mathrm{C} 9$ & $1.529(6)$ & $\mathrm{C} 42-\mathrm{C} 43$ & $1.513(7)$ \\
\hline $\mathrm{C} 8-\mathrm{H} 8 \mathrm{~A}$ & 0.9900 & $\mathrm{C} 42-\mathrm{H} 42$ & 1.0000 \\
\hline $\mathrm{C} 8-\mathrm{H} 8 \mathrm{~B}$ & 0.9900 & $\mathrm{C} 43-\mathrm{C} 44$ & $1.529(6)$ \\
\hline $\mathrm{C} 9-\mathrm{C} 10$ & $1.515(6)$ & $\mathrm{C} 43-\mathrm{H} 43 \mathrm{~A}$ & 0.9900 \\
\hline C9-H9A & 0.9900 & $\mathrm{C} 43-\mathrm{H} 43 \mathrm{~B}$ & 0.9900 \\
\hline С9-H9B & 0.9900 & $\mathrm{C} 44-\mathrm{C} 45$ & $1.504(6)$ \\
\hline
\end{tabular}




\begin{tabular}{|c|c|c|c|}
\hline $\mathrm{C} 10-\mathrm{C} 11$ & $1.515(7)$ & $\mathrm{C} 44-\mathrm{H} 44 \mathrm{~A}$ & 0.9900 \\
\hline $\mathrm{C} 10-\mathrm{H} 10 \mathrm{~A}$ & 0.9900 & $\mathrm{C} 44-\mathrm{H} 44 \mathrm{~B}$ & 0.9900 \\
\hline $\mathrm{C} 10-\mathrm{H} 10 \mathrm{~B}$ & 0.9900 & $\mathrm{C} 45-\mathrm{C} 46$ & $1.515(7)$ \\
\hline $\mathrm{C} 11-\mathrm{C} 12$ & $1.523(5)$ & $\mathrm{C} 45-\mathrm{H} 45 \mathrm{~A}$ & 0.9900 \\
\hline $\mathrm{C} 11-\mathrm{H} 11 \mathrm{~A}$ & 0.9900 & $\mathrm{C} 45-\mathrm{H} 45 \mathrm{~B}$ & 0.9900 \\
\hline C11-H11B & 0.9900 & $\mathrm{C} 46-\mathrm{C} 47$ & $1.522(6)$ \\
\hline $\mathrm{C} 12-\mathrm{H} 12 \mathrm{~A}$ & 0.9900 & $\mathrm{C} 46-\mathrm{H} 46 \mathrm{~A}$ & 0.9900 \\
\hline $\mathrm{C} 12-\mathrm{H} 12 \mathrm{~B}$ & 0.9900 & $\mathrm{C} 46-\mathrm{H} 46 \mathrm{~B}$ & 0.9900 \\
\hline $\mathrm{C} 13-\mathrm{C} 14$ & $1.511(6)$ & C47-H47A & 0.9900 \\
\hline $\mathrm{C} 13-\mathrm{C} 18$ & $1.526(6)$ & C47-H47B & 0.9900 \\
\hline $\mathrm{C} 13-\mathrm{H} 13$ & 1.0000 & $\mathrm{O} 1 \mathrm{~A}-\mathrm{C} 51 \mathrm{~A}$ & $1.418(9)$ \\
\hline $\mathrm{C} 14-\mathrm{C} 15$ & $1.542(6)$ & $\mathrm{O} 1 \mathrm{~A}-\mathrm{C} 48 \mathrm{~A}$ & $1.477(11)$ \\
\hline $\mathrm{C} 14-\mathrm{H} 14 \mathrm{~A}$ & 0.9900 & $\mathrm{C} 48 \mathrm{~A}-\mathrm{C} 49 \mathrm{~A}$ & $1.463(10)$ \\
\hline C14-H14B & 0.9900 & $\mathrm{C} 48 \mathrm{~A}-\mathrm{H} 48 \mathrm{~A}$ & 0.9900 \\
\hline $\mathrm{C} 15-\mathrm{C} 16$ & $1.520(7)$ & $\mathrm{C} 48 \mathrm{~A}-\mathrm{H} 48 \mathrm{~B}$ & 0.9900 \\
\hline $\mathrm{C} 15-\mathrm{H} 15 \mathrm{~A}$ & 0.9900 & $\mathrm{C} 49 \mathrm{~A}-\mathrm{C} 50 \mathrm{~A}$ & $1.462(11)$ \\
\hline C15-H15B & 0.9900 & $\mathrm{C} 49 \mathrm{~A}-\mathrm{H} 49 \mathrm{~A}$ & 0.9900 \\
\hline $\mathrm{C} 16-\mathrm{C} 17$ & $1.500(7)$ & $\mathrm{C} 49 \mathrm{~A}-\mathrm{H} 49 \mathrm{~B}$ & 0.9900 \\
\hline $\mathrm{C} 16-\mathrm{H} 16 \mathrm{~A}$ & 0.9900 & $\mathrm{C} 50 \mathrm{~A}-\mathrm{C} 51 \mathrm{~A}$ & $1.458(10)$ \\
\hline C16-H16B & 0.9900 & $\mathrm{C} 50 \mathrm{~A}-\mathrm{H} 50 \mathrm{~A}$ & 0.9900 \\
\hline $\mathrm{C} 17-\mathrm{C} 18$ & $1.527(6)$ & $\mathrm{C} 50 \mathrm{~A}-\mathrm{H} 50 \mathrm{~B}$ & 0.9900 \\
\hline C17-H17A & 0.9900 & $\mathrm{C} 51 \mathrm{~A}-\mathrm{H} 51 \mathrm{~A}$ & 0.9900 \\
\hline C17-H17B & 0.9900 & $\mathrm{C} 51 \mathrm{~A}-\mathrm{H} 51 \mathrm{~B}$ & 0.9900 \\
\hline $\mathrm{C} 18-\mathrm{H} 18 \mathrm{~A}$ & 0.9900 & $\mathrm{O} 1 \mathrm{~B}-\mathrm{C} 51 \mathrm{~B}$ & $1.411(14)$ \\
\hline C18-H18B & 0.9900 & $\mathrm{O} 1 \mathrm{~B}-\mathrm{C} 48 \mathrm{~B}$ & $1.471(15)$ \\
\hline $\mathrm{C} 19 \mathrm{~A}-\mathrm{C} 24 \mathrm{~A}$ & $1.515(8)$ & $\mathrm{C} 48 \mathrm{~B}-\mathrm{C} 49 \mathrm{~B}$ & $1.492(15)$ \\
\hline $\mathrm{C} 19 \mathrm{~A}-\mathrm{C} 20 \mathrm{~A}$ & $1.525(7)$ & $\mathrm{C} 48 \mathrm{~B}-\mathrm{H} 48 \mathrm{C}$ & 0.9900 \\
\hline $\mathrm{C} 19 \mathrm{~A}-\mathrm{H} 19 \mathrm{~A}$ & 1.0000 & $\mathrm{C} 48 \mathrm{~B}-\mathrm{H} 48 \mathrm{D}$ & 0.9900 \\
\hline $\mathrm{C} 20 \mathrm{~A}-\mathrm{C} 21 \mathrm{~A}$ & $1.527(8)$ & $\mathrm{C} 49 \mathrm{~B}-\mathrm{C} 50 \mathrm{~B}$ & $1.501(15)$ \\
\hline $\mathrm{C} 20 \mathrm{~A}-\mathrm{H} 20 \mathrm{~A}$ & 0.9900 & $\mathrm{C} 49 \mathrm{~B}-\mathrm{H} 49 \mathrm{C}$ & 0.9900 \\
\hline $\mathrm{C} 20 \mathrm{~A}-\mathrm{H} 20 \mathrm{~B}$ & 0.9900 & $\mathrm{C} 49 \mathrm{~B}-\mathrm{H} 49 \mathrm{D}$ & 0.9900 \\
\hline $\mathrm{C} 21 \mathrm{~A}-\mathrm{C} 22 \mathrm{~A}$ & $1.521(9)$ & $\mathrm{C} 50 \mathrm{~B}-\mathrm{C} 51 \mathrm{~B}$ & $1.491(16)$ \\
\hline $\mathrm{C} 21 \mathrm{~A}-\mathrm{H} 21 \mathrm{~A}$ & 0.9900 & $\mathrm{C} 50 \mathrm{~B}-\mathrm{H} 50 \mathrm{C}$ & 0.9900 \\
\hline $\mathrm{C} 21 \mathrm{~A}-\mathrm{H} 21 \mathrm{~B}$ & 0.9900 & $\mathrm{C} 50 \mathrm{~B}-\mathrm{H} 50 \mathrm{D}$ & 0.9900 \\
\hline $\mathrm{C} 22 \mathrm{~A}-\mathrm{C} 23 \mathrm{~A}$ & $1.504(9)$ & $\mathrm{C} 51 \mathrm{~B}-\mathrm{H} 51 \mathrm{C}$ & 0.9900 \\
\hline $\mathrm{C} 22 \mathrm{~A}-\mathrm{H} 22 \mathrm{~A}$ & 0.9900 & C51B-H51D & 0.9900 \\
\hline $\mathrm{C} 22 \mathrm{~A}-\mathrm{H} 22 \mathrm{~B}$ & 0.9900 & $\mathrm{O} 2 \mathrm{~A}-\mathrm{C} 55 \mathrm{~A}$ & $1.409(12)$ \\
\hline $\mathrm{C} 23 \mathrm{~A}-\mathrm{C} 24 \mathrm{~A}$ & $1.532(7)$ & $\mathrm{O} 2 \mathrm{~A}-\mathrm{C} 52 \mathrm{~A}$ & $1.452(9)$ \\
\hline $\mathrm{C} 23 \mathrm{~A}-\mathrm{H} 23 \mathrm{~A}$ & 0.9900 & $\mathrm{C} 52 \mathrm{~A}-\mathrm{C} 53 \mathrm{~A}$ & $1.466(10)$ \\
\hline $\mathrm{C} 23 \mathrm{~A}-\mathrm{H} 23 \mathrm{~B}$ & 0.9900 & $\mathrm{C} 52 \mathrm{~A}-\mathrm{H} 52 \mathrm{~A}$ & 0.9900 \\
\hline $\mathrm{C} 24 \mathrm{~A}-\mathrm{H} 24 \mathrm{~A}$ & 0.9900 & $\mathrm{C} 52 \mathrm{~A}-\mathrm{H} 52 \mathrm{~B}$ & 0.9900 \\
\hline $\mathrm{C} 24 \mathrm{~A}-\mathrm{H} 24 \mathrm{~B}$ & 0.9900 & $\mathrm{C} 53 \mathrm{~A}-\mathrm{C} 54 \mathrm{~A}$ & $1.455(10)$ \\
\hline $\mathrm{C} 19 \mathrm{~B}-\mathrm{C} 24 \mathrm{~B}$ & $1.504(16)$ & $\mathrm{C} 53 \mathrm{~A}-\mathrm{H} 53 \mathrm{~A}$ & 0.9900 \\
\hline $\mathrm{C} 19 \mathrm{~B}-\mathrm{C} 20 \mathrm{~B}$ & $1.521(16)$ & $\mathrm{C} 53 \mathrm{~A}-\mathrm{H} 53 \mathrm{~B}$ & 0.9900 \\
\hline $\mathrm{C} 19 \mathrm{~B}-\mathrm{H} 19 \mathrm{~B}$ & 1.0000 & $\mathrm{C} 54 \mathrm{~A}-\mathrm{C} 55 \mathrm{~A}$ & $1.442(9)$ \\
\hline $\mathrm{C} 20 \mathrm{~B}-\mathrm{C} 21 \mathrm{~B}$ & $1.523(17)$ & $\mathrm{C} 54 \mathrm{~A}-\mathrm{H} 54 \mathrm{~A}$ & 0.9900 \\
\hline $\mathrm{C} 20 \mathrm{~B}-\mathrm{H} 20 \mathrm{C}$ & 0.9900 & $\mathrm{C} 54 \mathrm{~A}-\mathrm{H} 54 \mathrm{~B}$ & 0.9900 \\
\hline $\mathrm{C} 20 \mathrm{~B}-\mathrm{H} 20 \mathrm{D}$ & 0.9900 & $\mathrm{C} 55 \mathrm{~A}-\mathrm{H} 55 \mathrm{~A}$ & 0.9900 \\
\hline
\end{tabular}




\begin{tabular}{|c|c|c|c|}
\hline $\mathrm{C} 21 \mathrm{~B}-\mathrm{C} 22 \mathrm{~B}$ & $1.510(17)$ & $\mathrm{C} 55 \mathrm{~A}-\mathrm{H} 55 \mathrm{~B}$ & 0.9900 \\
\hline $\mathrm{C} 21 \mathrm{~B}-\mathrm{H} 21 \mathrm{C}$ & 0.9900 & $\mathrm{O} 2 \mathrm{~B}-\mathrm{C} 55 \mathrm{~B}$ & $1.408(17)$ \\
\hline $\mathrm{C} 21 \mathrm{~B}-\mathrm{H} 21 \mathrm{D}$ & 0.9900 & $\mathrm{O} 2 \mathrm{~B}-\mathrm{C} 52 \mathrm{~B}$ & $1.465(18)$ \\
\hline $\mathrm{C} 22 \mathrm{~B}-\mathrm{C} 23 \mathrm{~B}$ & $1.500(17)$ & $\mathrm{C} 52 \mathrm{~B}-\mathrm{C} 53 \mathrm{~B}$ & $1.473(17)$ \\
\hline $\mathrm{C} 22 \mathrm{~B}-\mathrm{H} 22 \mathrm{C}$ & 0.9900 & $\mathrm{C} 52 \mathrm{~B}-\mathrm{H} 52 \mathrm{C}$ & 0.9900 \\
\hline $\mathrm{C} 22 \mathrm{~B}-\mathrm{H} 22 \mathrm{D}$ & 0.9900 & $\mathrm{C} 52 \mathrm{~B}-\mathrm{H} 52 \mathrm{D}$ & 0.9900 \\
\hline $\mathrm{C} 23 \mathrm{~B}-\mathrm{C} 24 \mathrm{~B}$ & $1.548(16)$ & $\mathrm{C} 53 \mathrm{~B}-\mathrm{C} 54 \mathrm{~B}$ & $1.455(17)$ \\
\hline $\mathrm{C} 23 \mathrm{~B}-\mathrm{H} 23 \mathrm{C}$ & 0.9900 & $\mathrm{C} 53 \mathrm{~B}-\mathrm{H} 53 \mathrm{C}$ & 0.9900 \\
\hline $\mathrm{C} 23 \mathrm{~B}-\mathrm{H} 23 \mathrm{D}$ & 0.9900 & $\mathrm{C} 53 \mathrm{~B}-\mathrm{H} 53 \mathrm{D}$ & 0.9900 \\
\hline $\mathrm{C} 24 \mathrm{~B}-\mathrm{H} 24 \mathrm{C}$ & 0.9900 & $\mathrm{C} 54 \mathrm{~B}-\mathrm{C} 55 \mathrm{~B}$ & $1.464(17)$ \\
\hline $\mathrm{C} 24 \mathrm{~B}-\mathrm{H} 24 \mathrm{D}$ & 0.9900 & $\mathrm{C} 54 \mathrm{~B}-\mathrm{H} 54 \mathrm{C}$ & 0.9900 \\
\hline $\mathrm{C} 25-\mathrm{C} 30$ & $1.529(7)$ & $\mathrm{C} 54 \mathrm{~B}-\mathrm{H} 54 \mathrm{D}$ & 0.9900 \\
\hline $\mathrm{C} 25-\mathrm{C} 26$ & $1.529(7)$ & $\mathrm{C} 55 \mathrm{~B}-\mathrm{H} 55 \mathrm{C}$ & 0.9900 \\
\hline $\mathrm{C} 25-\mathrm{H} 25$ & 1.0000 & $\mathrm{C} 55 \mathrm{~B}-\mathrm{H} 55 \mathrm{D}$ & 0.9900 \\
\hline $\mathrm{N} 5-\mathrm{HO}-\mathrm{N} 6$ & $57.33(11)$ & $\mathrm{C} 23 \mathrm{~B}-\mathrm{C} 24 \mathrm{~B}-\mathrm{H} 24 \mathrm{D}$ & 109.9 \\
\hline $\mathrm{N} 5-\mathrm{HO}-\mathrm{N} 2$ & $99.94(12)$ & $\mathrm{H} 24 \mathrm{C}-\mathrm{C} 24 \mathrm{~B}-\mathrm{H} 24 \mathrm{D}$ & 108.3 \\
\hline $\mathrm{N} 6-\mathrm{HO}-\mathrm{N} 2$ & $102.32(12)$ & $\mathrm{N} 4-\mathrm{C} 25-\mathrm{C} 30$ & $110.4(4)$ \\
\hline $\mathrm{N} 5-\mathrm{HO}-\mathrm{N} 1$ & $107.97(11)$ & $\mathrm{N} 4-\mathrm{C} 25-\mathrm{C} 26$ & $108.4(4)$ \\
\hline $\mathrm{N} 6-\mathrm{HO}-\mathrm{N} 1$ & $154.43(11)$ & $\mathrm{C} 30-\mathrm{C} 25-\mathrm{C} 26$ & $108.9(4)$ \\
\hline $\mathrm{N} 2-\mathrm{HO}-\mathrm{N} 1$ & $57.02(11)$ & $\mathrm{N} 4-\mathrm{C} 25-\mathrm{H} 25$ & 109.7 \\
\hline $\mathrm{N} 5-\mathrm{HO}-\mathrm{CL} 1$ & $154.44(8)$ & $\mathrm{C} 30-\mathrm{C} 25-\mathrm{H} 25$ & 109.7 \\
\hline $\mathrm{N} 6-\mathrm{HO}-\mathrm{CL} 1$ & $98.60(8)$ & $\mathrm{C} 26-\mathrm{C} 25-\mathrm{H} 25$ & 109.7 \\
\hline $\mathrm{N} 2-\mathrm{HO}-\mathrm{CL} 1$ & $93.27(9)$ & $\mathrm{C} 27-\mathrm{C} 26-\mathrm{C} 25$ & $111.8(4)$ \\
\hline $\mathrm{N} 1-\mathrm{HO}-\mathrm{CL} 1$ & $97.59(9)$ & $\mathrm{C} 27-\mathrm{C} 26-\mathrm{H} 26 \mathrm{~A}$ & 109.3 \\
\hline $\mathrm{N} 5-\mathrm{HO}-\mathrm{CL} 2$ & $92.29(9)$ & $\mathrm{C} 25-\mathrm{C} 26-\mathrm{H} 26 \mathrm{~A}$ & 109.3 \\
\hline $\mathrm{N} 6-\mathrm{HO}-\mathrm{CL} 2$ & $104.14(10)$ & $\mathrm{C} 27-\mathrm{C} 26-\mathrm{H} 26 \mathrm{~B}$ & 109.3 \\
\hline $\mathrm{N} 2-\mathrm{HO}-\mathrm{CL} 2$ & $153.43(8)$ & $\mathrm{C} 25-\mathrm{C} 26-\mathrm{H} 26 \mathrm{~B}$ & 109.3 \\
\hline $\mathrm{N} 1-\mathrm{HO}-\mathrm{CL} 2$ & $96.82(9)$ & $\mathrm{H} 26 \mathrm{~A}-\mathrm{C} 26-\mathrm{H} 26 \mathrm{~B}$ & 107.9 \\
\hline $\mathrm{CL} 1-\mathrm{HO}-\mathrm{CL} 2$ & $85.19(5)$ & $\mathrm{C} 28-\mathrm{C} 27-\mathrm{C} 26$ & $110.7(5)$ \\
\hline $\mathrm{N} 5-\mathrm{HO}-\mathrm{C} 31$ & $28.81(10)$ & $\mathrm{C} 28-\mathrm{C} 27-\mathrm{H} 27 \mathrm{~A}$ & 109.5 \\
\hline $\mathrm{N} 6-\mathrm{HO}-\mathrm{C} 31$ & $28.78(11)$ & $\mathrm{C} 26-\mathrm{C} 27-\mathrm{H} 27 \mathrm{~A}$ & 109.5 \\
\hline $\mathrm{N} 2-\mathrm{HO}-\mathrm{C} 31$ & $105.55(12)$ & $\mathrm{C} 28-\mathrm{C} 27-\mathrm{H} 27 \mathrm{~B}$ & 109.5 \\
\hline $\mathrm{N} 1-\mathrm{HO}-\mathrm{C} 31$ & $135.06(11)$ & $\mathrm{C} 26-\mathrm{C} 27-\mathrm{H} 27 \mathrm{~B}$ & 109.5 \\
\hline $\mathrm{CL} 1-\mathrm{HO}-\mathrm{C} 31$ & $126.18(9)$ & $\mathrm{H} 27 \mathrm{~A}-\mathrm{C} 27-\mathrm{H} 27 \mathrm{~B}$ & 108.1 \\
\hline $\mathrm{CL} 2-\mathrm{HO}-\mathrm{C} 31$ & $96.54(10)$ & $\mathrm{C} 27-\mathrm{C} 28-\mathrm{C} 29$ & $111.5(4)$ \\
\hline $\mathrm{N} 5-\mathrm{HO}-\mathrm{C} 1$ & $107.74(12)$ & $\mathrm{C} 27-\mathrm{C} 28-\mathrm{H} 28 \mathrm{~A}$ & 109.3 \\
\hline $\mathrm{N} 6-\mathrm{HO}-\mathrm{C} 1$ & $130.11(12)$ & $\mathrm{C} 29-\mathrm{C} 28-\mathrm{H} 28 \mathrm{~A}$ & 109.3 \\
\hline $\mathrm{N} 2-\mathrm{HO}-\mathrm{C} 1$ & $28.64(11)$ & $\mathrm{C} 27-\mathrm{C} 28-\mathrm{H} 28 \mathrm{~B}$ & 109.3 \\
\hline $\mathrm{N} 1-\mathrm{HO}-\mathrm{C} 1$ & $28.49(11)$ & $\mathrm{C} 29-\mathrm{C} 28-\mathrm{H} 28 \mathrm{~B}$ & 109.3 \\
\hline $\mathrm{CL} 1-\mathrm{HO}-\mathrm{C} 1$ & $94.33(9)$ & $\mathrm{H} 28 \mathrm{~A}-\mathrm{C} 28-\mathrm{H} 28 \mathrm{~B}$ & 108.0 \\
\hline $\mathrm{CL} 2-\mathrm{HO}-\mathrm{C} 1$ & $124.91(9)$ & $\mathrm{C} 28-\mathrm{C} 29-\mathrm{C} 30$ & $111.7(4)$ \\
\hline $\mathrm{C} 31-\mathrm{HO}-\mathrm{C} 1$ & $125.31(12)$ & $\mathrm{C} 28-\mathrm{C} 29-\mathrm{H} 29 \mathrm{~A}$ & 109.3 \\
\hline $\mathrm{N} 5-\mathrm{HO}-\mathrm{LI}$ & $128.60(19)$ & $\mathrm{C} 30-\mathrm{C} 29-\mathrm{H} 29 \mathrm{~A}$ & 109.3 \\
\hline $\mathrm{N} 6-\mathrm{HO}-\mathrm{LI}$ & $104.43(17)$ & $\mathrm{C} 28-\mathrm{C} 29-\mathrm{H} 29 \mathrm{~B}$ & 109.3 \\
\hline $\mathrm{N} 2-\mathrm{HO}-\mathrm{LI}$ & $131.46(19)$ & $\mathrm{C} 30-\mathrm{C} 29-\mathrm{H} 29 \mathrm{~B}$ & 109.3 \\
\hline $\mathrm{N} 1-\mathrm{HO}-\mathrm{LI}$ & $100.94(17)$ & $\mathrm{H} 29 \mathrm{~A}-\mathrm{C} 29-\mathrm{H} 29 \mathrm{~B}$ & 107.9 \\
\hline $\mathrm{CL} 1-\mathrm{HO}-\mathrm{LI}$ & $43.27(18)$ & $\mathrm{C} 29-\mathrm{C} 30-\mathrm{C} 25$ & $111.7(4)$ \\
\hline
\end{tabular}




$\begin{array}{ll}\text { CL2-HO-LI } & 41.95(18) \\ \text { C31-HO-LI } & 117.28(18) \\ \text { C1-HO-LI } & 117.41(18) \\ \text { LI-CL1-HO } & 87.0(2) \\ \text { LI-CL2-HO } & 88.0(3) \\ \text { O2B-LI-O1B } & 97(3) \\ \text { O2A-LI-O1A } & 111.1(7) \\ \text { O2B-LI-CL2 } & 113.7(17) \\ \text { O1B-LI-CL2 } & 116.8(7) \\ \text { O2A-LI-CL2 } & 116.6(6) \\ \text { O1A-LI-CL2 } & 118.3(5) \\ \text { O2B-LI-CL1 } & 115(3) \\ \text { O1B-LI-CL1 } & 116.3(7) \\ \text { O2A-LI-CL1 } & 110.5(7) \\ \text { O1A-LI-CL1 } & 97.8(5) \\ \text { CL2-LI-CL1 } & 99.7(3) \\ \text { O2B-LI-HO } & 131(3) \\ \text { O1B-LI-HO } & 132.5(6) \\ \text { O2A-LI-HO } & 129.7(7) \\ \text { O1A-LI-HO } & 116.8(4) \\ \text { CL2-LI-HO } & 50.07(15) \\ \text { CL1-LI-HO } & 49.70(15) \\ \text { C1-N1-C7 } & 121.6(3) \\ \text { C1-N1-HO } & 93.5(2) \\ \text { C7-N1-HO } & 140.0(3) \\ \text { C1-N2-C13 } & 123.8(4) \\ \text { C1-N2-HO } & 93.9(2) \\ \text { C13-N2-HO } & 142.2(3) \\ \text { C2-N3-C1 } & 119.9(3) \\ \text { C2-N3-C19B } & 131.9(10) \\ \text { C1-N3-C19B } & 107.1(10) \\ \text { C2-N3-C19A } & 113.8(4) \\ \text { C1-N3-C19A } & 119.0(4) \\ \text { C2-N4-C25 } & 121.5(4) \\ \text { C31-N5-C36 } & 123.8(3) \\ \text { C31-N5-HO } & 94.2(2) \\ \text { C36-N5-HO } & 141.7(2) \\ \text { C31-N6-C42 } & 123.3(4) \\ \text { C31-N6-HO } & 93.6(2) \\ \text { C42-N6-HO } & 140.3(3) \\ \text { N1-C1-N2 } & 115.2(4) \\ \text { N1-C1-N3 } & 121.9(3) \\ \text { N2-C1-N3 } & 122.9(4) \\ \text { N1-C1-HO } & 58.0(2) \\ \text { N2-C1-HO } & 57.5(2) \\ \text { N3-C1-HO } & \\ \text { N4-C2-N3 } & \\ \text { N4-C2-C3 } & \\ & \\ & \end{array}$

\begin{tabular}{|c|c|}
\hline $\mathrm{C} 29-\mathrm{C} 30-\mathrm{H} 30 \mathrm{~A}$ & 109.3 \\
\hline $\mathrm{C} 25-\mathrm{C} 30-\mathrm{H} 30 \mathrm{~A}$ & 109.3 \\
\hline $\mathrm{C} 29-\mathrm{C} 30-\mathrm{H} 30 \mathrm{~B}$ & 109.3 \\
\hline $\mathrm{C} 25-\mathrm{C} 30-\mathrm{H} 30 \mathrm{~B}$ & 109.3 \\
\hline $\mathrm{H} 30 \mathrm{~A}-\mathrm{C} 30-\mathrm{H} 30 \mathrm{~B}$ & 107.9 \\
\hline $\mathrm{N} 6-\mathrm{C} 31-\mathrm{N} 5$ & $113.9(4)$ \\
\hline $\mathrm{N} 6-\mathrm{C} 31-\mathrm{C} 32$ & $122.9(3)$ \\
\hline $\mathrm{N} 5-\mathrm{C} 31-\mathrm{C} 32$ & $123.2(4)$ \\
\hline $\mathrm{N} 6-\mathrm{C} 31-\mathrm{HO}$ & $57.6(2)$ \\
\hline $\mathrm{N} 5-\mathrm{C} 31-\mathrm{HO}$ & $57.0(2)$ \\
\hline $\mathrm{C} 32-\mathrm{C} 31-\mathrm{HO}$ & $172.5(3)$ \\
\hline $\mathrm{C} 31-\mathrm{C} 32-\mathrm{C} 33$ & $110.7(4)$ \\
\hline $\mathrm{C} 31-\mathrm{C} 32-\mathrm{H} 32 \mathrm{~A}$ & 109.5 \\
\hline $\mathrm{C} 33-\mathrm{C} 32-\mathrm{H} 32 \mathrm{~A}$ & 109.5 \\
\hline $\mathrm{C} 31-\mathrm{C} 32-\mathrm{H} 32 \mathrm{~B}$ & 109.5 \\
\hline $\mathrm{C} 33-\mathrm{C} 32-\mathrm{H} 32 \mathrm{~B}$ & 109.5 \\
\hline $\mathrm{H} 32 \mathrm{~A}-\mathrm{C} 32-\mathrm{H} 32 \mathrm{~B}$ & 108.1 \\
\hline $\mathrm{C} 32-\mathrm{C} 33-\mathrm{C} 34$ & $113.6(4)$ \\
\hline $\mathrm{C} 32-\mathrm{C} 33-\mathrm{H} 33 \mathrm{~A}$ & 108.8 \\
\hline $\mathrm{C} 34-\mathrm{C} 33-\mathrm{H} 33 \mathrm{~A}$ & 108.8 \\
\hline С $32-\mathrm{C} 33-\mathrm{H} 33 \mathrm{~B}$ & 108.8 \\
\hline C $34-\mathrm{C} 33-\mathrm{H} 33 \mathrm{~B}$ & 108.8 \\
\hline $\mathrm{H} 33 \mathrm{~A}-\mathrm{C} 33-\mathrm{H} 33 \mathrm{~B}$ & 107.7 \\
\hline $\mathrm{C} 35-\mathrm{C} 34-\mathrm{C} 33$ & $113.1(4)$ \\
\hline $\mathrm{C} 35-\mathrm{C} 34-\mathrm{H} 34 \mathrm{~A}$ & 109.0 \\
\hline $\mathrm{C} 33-\mathrm{C} 34-\mathrm{H} 34 \mathrm{~A}$ & 109.0 \\
\hline $\mathrm{C} 35-\mathrm{C} 34-\mathrm{H} 34 \mathrm{~B}$ & 109.0 \\
\hline C33-C34-H34B & 109.0 \\
\hline $\mathrm{H} 34 \mathrm{~A}-\mathrm{C} 34-\mathrm{H} 34 \mathrm{~B}$ & 107.8 \\
\hline $\mathrm{C} 34-\mathrm{C} 35-\mathrm{H} 35 \mathrm{~A}$ & 109.5 \\
\hline $\mathrm{C} 34-\mathrm{C} 35-\mathrm{H} 35 \mathrm{~B}$ & 109.5 \\
\hline $\mathrm{H} 35 \mathrm{~A}-\mathrm{C} 35-\mathrm{H} 35 \mathrm{~B}$ & 109.5 \\
\hline $\mathrm{C} 34-\mathrm{C} 35-\mathrm{H} 35 \mathrm{C}$ & 109.5 \\
\hline $\mathrm{H} 35 \mathrm{~A}-\mathrm{C} 35-\mathrm{H} 35 \mathrm{C}$ & 109.5 \\
\hline $\mathrm{H} 35 \mathrm{~B}-\mathrm{C} 35-\mathrm{H} 35 \mathrm{C}$ & 109.5 \\
\hline $\mathrm{N} 5-\mathrm{C} 36-\mathrm{C} 37$ & $111.0(4)$ \\
\hline $\mathrm{N} 5-\mathrm{C} 36-\mathrm{C} 41$ & $109.7(3)$ \\
\hline $\mathrm{C} 37-\mathrm{C} 36-\mathrm{C} 41$ & $109.8(4)$ \\
\hline $\mathrm{N} 5-\mathrm{C} 36-\mathrm{H} 36$ & 108.8 \\
\hline $\mathrm{C} 37-\mathrm{C} 36-\mathrm{H} 36$ & 108.8 \\
\hline $\mathrm{C} 41-\mathrm{C} 36-\mathrm{H} 36$ & 108.8 \\
\hline $\mathrm{C} 36-\mathrm{C} 37-\mathrm{C} 38$ & $113.5(4)$ \\
\hline $\mathrm{C} 36-\mathrm{C} 37-\mathrm{H} 37 \mathrm{~A}$ & 108.9 \\
\hline $\mathrm{C} 38-\mathrm{C} 37-\mathrm{H} 37 \mathrm{~A}$ & 108.9 \\
\hline $\mathrm{C} 36-\mathrm{C} 37-\mathrm{H} 37 \mathrm{~B}$ & 108.9 \\
\hline С $38-\mathrm{C} 37-\mathrm{H} 37 \mathrm{~B}$ & 108.9 \\
\hline $\mathrm{H} 37 \mathrm{~A}-\mathrm{C} 37-\mathrm{H} 37 \mathrm{~B}$ & 107.7 \\
\hline $\mathrm{C} 39-\mathrm{C} 38-\mathrm{C} 37$ & $110.3(4)$ \\
\hline
\end{tabular}




\begin{tabular}{|c|c|c|c|}
\hline $\mathrm{N} 3-\mathrm{C} 2-\mathrm{C} 3$ & $114.2(4)$ & $\mathrm{C} 39-\mathrm{C} 38-\mathrm{H} 38 \mathrm{~A}$ & 109.6 \\
\hline $\mathrm{C} 2-\mathrm{C} 3-\mathrm{C} 4$ & $113.6(3)$ & $\mathrm{C} 37-\mathrm{C} 38-\mathrm{H} 38 \mathrm{~A}$ & 109.6 \\
\hline $\mathrm{C} 2-\mathrm{C} 3-\mathrm{H} 3 \mathrm{~A}$ & 108.8 & $\mathrm{C} 39-\mathrm{C} 38-\mathrm{H} 38 \mathrm{~B}$ & 109.6 \\
\hline $\mathrm{C} 4-\mathrm{C} 3-\mathrm{H} 3 \mathrm{~A}$ & 108.8 & $\mathrm{C} 37-\mathrm{C} 38-\mathrm{H} 38 \mathrm{~B}$ & 109.6 \\
\hline $\mathrm{C} 2-\mathrm{C} 3-\mathrm{H} 3 \mathrm{~B}$ & 108.8 & $\mathrm{H} 38 \mathrm{~A}-\mathrm{C} 38-\mathrm{H} 38 \mathrm{~B}$ & 108.1 \\
\hline $\mathrm{C} 4-\mathrm{C} 3-\mathrm{H} 3 \mathrm{~B}$ & 108.8 & $\mathrm{C} 40-\mathrm{C} 39-\mathrm{C} 38$ & $110.9(4)$ \\
\hline $\mathrm{H} 3 \mathrm{~A}-\mathrm{C} 3-\mathrm{H} 3 \mathrm{~B}$ & 107.7 & $\mathrm{C} 40-\mathrm{C} 39-\mathrm{H} 39 \mathrm{~A}$ & 109.5 \\
\hline $\mathrm{C} 5-\mathrm{C} 4-\mathrm{C} 3$ & $110.8(3)$ & $\mathrm{C} 38-\mathrm{C} 39-\mathrm{H} 39 \mathrm{~A}$ & 109.5 \\
\hline $\mathrm{C} 5-\mathrm{C} 4-\mathrm{H} 4 \mathrm{~A}$ & 109.5 & $\mathrm{C} 40-\mathrm{C} 39-\mathrm{H} 39 \mathrm{~B}$ & 109.5 \\
\hline $\mathrm{C} 3-\mathrm{C} 4-\mathrm{H} 4 \mathrm{~A}$ & 109.5 & $\mathrm{C} 38-\mathrm{C} 39-\mathrm{H} 39 \mathrm{~B}$ & 109.5 \\
\hline $\mathrm{C} 5-\mathrm{C} 4-\mathrm{H} 4 \mathrm{~B}$ & 109.5 & $\mathrm{H} 39 \mathrm{~A}-\mathrm{C} 39-\mathrm{H} 39 \mathrm{~B}$ & 108.0 \\
\hline $\mathrm{C} 3-\mathrm{C} 4-\mathrm{H} 4 \mathrm{~B}$ & 109.5 & $\mathrm{C} 39-\mathrm{C} 40-\mathrm{C} 41$ & $112.5(4)$ \\
\hline $\mathrm{H} 4 \mathrm{~A}-\mathrm{C} 4-\mathrm{H} 4 \mathrm{~B}$ & 108.1 & $\mathrm{C} 39-\mathrm{C} 40-\mathrm{H} 40 \mathrm{~A}$ & 109.1 \\
\hline $\mathrm{C} 4-\mathrm{C} 5-\mathrm{C} 6$ & $113.7(4)$ & $\mathrm{C} 41-\mathrm{C} 40-\mathrm{H} 40 \mathrm{~A}$ & 109.1 \\
\hline $\mathrm{C} 4-\mathrm{C} 5-\mathrm{H} 5 \mathrm{~A}$ & 108.8 & $\mathrm{C} 39-\mathrm{C} 40-\mathrm{H} 40 \mathrm{~B}$ & 109.1 \\
\hline $\mathrm{C} 6-\mathrm{C} 5-\mathrm{H} 5 \mathrm{~A}$ & 108.8 & $\mathrm{C} 41-\mathrm{C} 40-\mathrm{H} 40 \mathrm{~B}$ & 109.1 \\
\hline $\mathrm{C} 4-\mathrm{C} 5-\mathrm{H} 5 \mathrm{~B}$ & 108.8 & $\mathrm{H} 40 \mathrm{~A}-\mathrm{C} 40-\mathrm{H} 40 \mathrm{~B}$ & 107.8 \\
\hline $\mathrm{C} 6-\mathrm{C} 5-\mathrm{H} 5 \mathrm{~B}$ & 108.8 & $\mathrm{C} 36-\mathrm{C} 41-\mathrm{C} 40$ & $111.2(4)$ \\
\hline $\mathrm{H} 5 \mathrm{~A}-\mathrm{C} 5-\mathrm{H} 5 \mathrm{~B}$ & 107.7 & $\mathrm{C} 36-\mathrm{C} 41-\mathrm{H} 41 \mathrm{~A}$ & 109.4 \\
\hline $\mathrm{C} 5-\mathrm{C} 6-\mathrm{H} 6 \mathrm{~A}$ & 109.5 & $\mathrm{C} 40-\mathrm{C} 41-\mathrm{H} 41 \mathrm{~A}$ & 109.4 \\
\hline $\mathrm{C} 5-\mathrm{C} 6-\mathrm{H} 6 \mathrm{~B}$ & 109.5 & $\mathrm{C} 36-\mathrm{C} 41-\mathrm{H} 41 \mathrm{~B}$ & 109.4 \\
\hline $\mathrm{H} 6 \mathrm{~A}-\mathrm{C} 6-\mathrm{H} 6 \mathrm{~B}$ & 109.5 & $\mathrm{C} 40-\mathrm{C} 41-\mathrm{H} 41 \mathrm{~B}$ & 109.4 \\
\hline $\mathrm{C} 5-\mathrm{C} 6-\mathrm{H} 6 \mathrm{C}$ & 109.5 & $\mathrm{H} 41 \mathrm{~A}-\mathrm{C} 41-\mathrm{H} 41 \mathrm{~B}$ & 108.0 \\
\hline $\mathrm{H} 6 \mathrm{~A}-\mathrm{C} 6-\mathrm{H} 6 \mathrm{C}$ & 109.5 & $\mathrm{~N} 6-\mathrm{C} 42-\mathrm{C} 47$ & $110.7(4)$ \\
\hline $\mathrm{H} 6 \mathrm{~B}-\mathrm{C} 6-\mathrm{H} 6 \mathrm{C}$ & 109.5 & $\mathrm{~N} 6-\mathrm{C} 42-\mathrm{C} 43$ & $108.9(4)$ \\
\hline $\mathrm{N} 1-\mathrm{C} 7-\mathrm{C} 12$ & $111.1(3)$ & $\mathrm{C} 47-\mathrm{C} 42-\mathrm{C} 43$ & $111.3(4)$ \\
\hline $\mathrm{N} 1-\mathrm{C} 7-\mathrm{C} 8$ & $110.5(3)$ & $\mathrm{N} 6-\mathrm{C} 42-\mathrm{H} 42$ & 108.6 \\
\hline $\mathrm{C} 12-\mathrm{C} 7-\mathrm{C} 8$ & $109.0(4)$ & $\mathrm{C} 47-\mathrm{C} 42-\mathrm{H} 42$ & 108.6 \\
\hline $\mathrm{N} 1-\mathrm{C} 7-\mathrm{H} 7$ & 108.7 & $\mathrm{C} 43-\mathrm{C} 42-\mathrm{H} 42$ & 108.6 \\
\hline $\mathrm{C} 12-\mathrm{C} 7-\mathrm{H} 7$ & 108.7 & $\mathrm{C} 42-\mathrm{C} 43-\mathrm{C} 44$ & $111.5(4)$ \\
\hline $\mathrm{C} 8-\mathrm{C} 7-\mathrm{H} 7$ & 108.7 & $\mathrm{C} 42-\mathrm{C} 43-\mathrm{H} 43 \mathrm{~A}$ & 109.3 \\
\hline $\mathrm{C} 7-\mathrm{C} 8-\mathrm{C} 9$ & $110.8(4)$ & $\mathrm{C} 44-\mathrm{C} 43-\mathrm{H} 43 \mathrm{~A}$ & 109.3 \\
\hline $\mathrm{C} 7-\mathrm{C} 8-\mathrm{H} 8 \mathrm{~A}$ & 109.5 & $\mathrm{C} 42-\mathrm{C} 43-\mathrm{H} 43 \mathrm{~B}$ & 109.3 \\
\hline $\mathrm{C} 9-\mathrm{C} 8-\mathrm{H} 8 \mathrm{~A}$ & 109.5 & $\mathrm{C} 44-\mathrm{C} 43-\mathrm{H} 43 \mathrm{~B}$ & 109.3 \\
\hline $\mathrm{C} 7-\mathrm{C} 8-\mathrm{H} 8 \mathrm{~B}$ & 109.5 & $\mathrm{H} 43 \mathrm{~A}-\mathrm{C} 43-\mathrm{H} 43 \mathrm{~B}$ & 108.0 \\
\hline $\mathrm{C} 9-\mathrm{C} 8-\mathrm{H} 8 \mathrm{~B}$ & 109.5 & $\mathrm{C} 45-\mathrm{C} 44-\mathrm{C} 43$ & $111.2(4)$ \\
\hline $\mathrm{H} 8 \mathrm{~A}-\mathrm{C} 8-\mathrm{H} 8 \mathrm{~B}$ & 108.1 & $\mathrm{C} 45-\mathrm{C} 44-\mathrm{H} 44 \mathrm{~A}$ & 109.4 \\
\hline $\mathrm{C} 10-\mathrm{C} 9-\mathrm{C} 8$ & $111.8(4)$ & $\mathrm{C} 43-\mathrm{C} 44-\mathrm{H} 44 \mathrm{~A}$ & 109.4 \\
\hline $\mathrm{C} 10-\mathrm{C} 9-\mathrm{H} 9 \mathrm{~A}$ & 109.3 & $\mathrm{C} 45-\mathrm{C} 44-\mathrm{H} 44 \mathrm{~B}$ & 109.4 \\
\hline $\mathrm{C} 8-\mathrm{C} 9-\mathrm{H} 9 \mathrm{~A}$ & 109.3 & $\mathrm{C} 43-\mathrm{C} 44-\mathrm{H} 44 \mathrm{~B}$ & 109.4 \\
\hline $\mathrm{C} 10-\mathrm{C} 9-\mathrm{H} 9 \mathrm{~B}$ & 109.3 & $\mathrm{H} 44 \mathrm{~A}-\mathrm{C} 44-\mathrm{H} 44 \mathrm{~B}$ & 108.0 \\
\hline $\mathrm{C} 8-\mathrm{C} 9-\mathrm{H} 9 \mathrm{~B}$ & 109.3 & $\mathrm{C} 44-\mathrm{C} 45-\mathrm{C} 46$ & $110.8(4)$ \\
\hline $\mathrm{H} 9 \mathrm{~A}-\mathrm{C} 9-\mathrm{H} 9 \mathrm{~B}$ & 107.9 & $\mathrm{C} 44-\mathrm{C} 45-\mathrm{H} 45 \mathrm{~A}$ & 109.5 \\
\hline $\mathrm{C} 11-\mathrm{C} 10-\mathrm{C} 9$ & $111.2(4)$ & $\mathrm{C} 46-\mathrm{C} 45-\mathrm{H} 45 \mathrm{~A}$ & 109.5 \\
\hline $\mathrm{C} 11-\mathrm{C} 10-\mathrm{H} 10 \mathrm{~A}$ & 109.4 & $\mathrm{C} 44-\mathrm{C} 45-\mathrm{H} 45 \mathrm{~B}$ & 109.5 \\
\hline $\mathrm{C} 9-\mathrm{C} 10-\mathrm{H} 10 \mathrm{~A}$ & 109.4 & $\mathrm{C} 46-\mathrm{C} 45-\mathrm{H} 45 \mathrm{~B}$ & 109.5 \\
\hline $\mathrm{C} 11-\mathrm{C} 10-\mathrm{H} 10 \mathrm{~B}$ & 109.4 & $\mathrm{H} 45 \mathrm{~A}-\mathrm{C} 45-\mathrm{H} 45 \mathrm{~B}$ & 108.1 \\
\hline $\mathrm{C} 9-\mathrm{C} 10-\mathrm{H} 10 \mathrm{~B}$ & 109.4 & $\mathrm{C} 45-\mathrm{C} 46-\mathrm{C} 47$ & $111.4(4)$ \\
\hline
\end{tabular}




\begin{tabular}{|c|c|c|c|}
\hline $\mathrm{H} 10 \mathrm{~A}-\mathrm{C} 10-\mathrm{H} 10 \mathrm{~B}$ & 108.0 & $\mathrm{C} 45-\mathrm{C} 46-\mathrm{H} 46 \mathrm{~A}$ & 109.4 \\
\hline $\mathrm{C} 10-\mathrm{C} 11-\mathrm{C} 12$ & $110.7(4)$ & $\mathrm{C} 47-\mathrm{C} 46-\mathrm{H} 46 \mathrm{~A}$ & 109.4 \\
\hline $\mathrm{C} 10-\mathrm{C} 11-\mathrm{H} 11 \mathrm{~A}$ & 109.5 & $\mathrm{C} 45-\mathrm{C} 46-\mathrm{H} 46 \mathrm{~B}$ & 109.4 \\
\hline $\mathrm{C} 12-\mathrm{C} 11-\mathrm{H} 11 \mathrm{~A}$ & 109.5 & $\mathrm{C} 47-\mathrm{C} 46-\mathrm{H} 46 \mathrm{~B}$ & 109.4 \\
\hline $\mathrm{C} 10-\mathrm{C} 11-\mathrm{H} 11 \mathrm{~B}$ & 109.5 & $\mathrm{H} 46 \mathrm{~A}-\mathrm{C} 46-\mathrm{H} 46 \mathrm{~B}$ & 108.0 \\
\hline $\mathrm{C} 12-\mathrm{C} 11-\mathrm{H} 11 \mathrm{~B}$ & 109.5 & $\mathrm{C} 42-\mathrm{C} 47-\mathrm{C} 46$ & $112.0(4)$ \\
\hline $\mathrm{H} 11 \mathrm{~A}-\mathrm{C} 11-\mathrm{H} 11 \mathrm{~B}$ & 108.1 & $\mathrm{C} 42-\mathrm{C} 47-\mathrm{H} 47 \mathrm{~A}$ & 109.2 \\
\hline $\mathrm{C} 7-\mathrm{C} 12-\mathrm{C} 11$ & $111.4(3)$ & $\mathrm{C} 46-\mathrm{C} 47-\mathrm{H} 47 \mathrm{~A}$ & 109.2 \\
\hline $\mathrm{C} 7-\mathrm{C} 12-\mathrm{H} 12 \mathrm{~A}$ & 109.3 & $\mathrm{C} 42-\mathrm{C} 47-\mathrm{H} 47 \mathrm{~B}$ & 109.2 \\
\hline $\mathrm{C} 11-\mathrm{C} 12-\mathrm{H} 12 \mathrm{~A}$ & 109.3 & $\mathrm{C} 46-\mathrm{C} 47-\mathrm{H} 47 \mathrm{~B}$ & 109.2 \\
\hline $\mathrm{C} 7-\mathrm{C} 12-\mathrm{H} 12 \mathrm{~B}$ & 109.3 & $\mathrm{H} 47 \mathrm{~A}-\mathrm{C} 47-\mathrm{H} 47 \mathrm{~B}$ & 107.9 \\
\hline $\mathrm{C} 11-\mathrm{C} 12-\mathrm{H} 12 \mathrm{~B}$ & 109.3 & $\mathrm{C} 51 \mathrm{~A}-\mathrm{O} 1 \mathrm{~A}-\mathrm{C} 48 \mathrm{~A}$ & $110.3(6)$ \\
\hline $\mathrm{H} 12 \mathrm{~A}-\mathrm{C} 12-\mathrm{H} 12 \mathrm{~B}$ & 108.0 & $\mathrm{C} 51 \mathrm{~A}-\mathrm{O} 1 \mathrm{~A}-\mathrm{LI}$ & $123.6(6)$ \\
\hline $\mathrm{N} 2-\mathrm{C} 13-\mathrm{C} 14$ & $110.4(3)$ & $\mathrm{C} 48 \mathrm{~A}-\mathrm{O} 1 \mathrm{~A}-\mathrm{LI}$ & $123.8(7)$ \\
\hline $\mathrm{N} 2-\mathrm{C} 13-\mathrm{C} 18$ & $108.7(4)$ & $\mathrm{C} 49 \mathrm{~A}-\mathrm{C} 48 \mathrm{~A}-\mathrm{O} 1 \mathrm{~A}$ & $104.2(8)$ \\
\hline $\mathrm{C} 14-\mathrm{C} 13-\mathrm{C} 18$ & $109.2(3)$ & $\mathrm{C} 49 \mathrm{~A}-\mathrm{C} 48 \mathrm{~A}-\mathrm{H} 48 \mathrm{~A}$ & 110.9 \\
\hline $\mathrm{N} 2-\mathrm{C} 13-\mathrm{H} 13$ & 109.5 & $\mathrm{O} 1 \mathrm{~A}-\mathrm{C} 48 \mathrm{~A}-\mathrm{H} 48 \mathrm{~A}$ & 110.9 \\
\hline $\mathrm{C} 14-\mathrm{C} 13-\mathrm{H} 13$ & 109.5 & $\mathrm{C} 49 \mathrm{~A}-\mathrm{C} 48 \mathrm{~A}-\mathrm{H} 48 \mathrm{~B}$ & 110.9 \\
\hline $\mathrm{C} 18-\mathrm{C} 13-\mathrm{H} 13$ & 109.5 & $\mathrm{O} 1 \mathrm{~A}-\mathrm{C} 48 \mathrm{~A}-\mathrm{H} 48 \mathrm{~B}$ & 110.9 \\
\hline $\mathrm{C} 13-\mathrm{C} 14-\mathrm{C} 15$ & $111.5(4)$ & $\mathrm{H} 48 \mathrm{~A}-\mathrm{C} 48 \mathrm{~A}-\mathrm{H} 48 \mathrm{~B}$ & 108.9 \\
\hline $\mathrm{C} 13-\mathrm{C} 14-\mathrm{H} 14 \mathrm{~A}$ & 109.3 & $\mathrm{C} 50 \mathrm{~A}-\mathrm{C} 49 \mathrm{~A}-\mathrm{C} 48 \mathrm{~A}$ & $105.5(8)$ \\
\hline $\mathrm{C} 15-\mathrm{C} 14-\mathrm{H} 14 \mathrm{~A}$ & 109.3 & $\mathrm{C} 50 \mathrm{~A}-\mathrm{C} 49 \mathrm{~A}-\mathrm{H} 49 \mathrm{~A}$ & 110.6 \\
\hline $\mathrm{C} 13-\mathrm{C} 14-\mathrm{H} 14 \mathrm{~B}$ & 109.3 & $\mathrm{C} 48 \mathrm{~A}-\mathrm{C} 49 \mathrm{~A}-\mathrm{H} 49 \mathrm{~A}$ & 110.6 \\
\hline $\mathrm{C} 15-\mathrm{C} 14-\mathrm{H} 14 \mathrm{~B}$ & 109.3 & $\mathrm{C} 50 \mathrm{~A}-\mathrm{C} 49 \mathrm{~A}-\mathrm{H} 49 \mathrm{~B}$ & 110.6 \\
\hline $\mathrm{H} 14 \mathrm{~A}-\mathrm{C} 14-\mathrm{H} 14 \mathrm{~B}$ & 108.0 & $\mathrm{C} 48 \mathrm{~A}-\mathrm{C} 49 \mathrm{~A}-\mathrm{H} 49 \mathrm{~B}$ & 110.6 \\
\hline $\mathrm{C} 16-\mathrm{C} 15-\mathrm{C} 14$ & $109.7(4)$ & $\mathrm{H} 49 \mathrm{~A}-\mathrm{C} 49 \mathrm{~A}-\mathrm{H} 49 \mathrm{~B}$ & 108.8 \\
\hline $\mathrm{C} 16-\mathrm{C} 15-\mathrm{H} 15 \mathrm{~A}$ & 109.7 & $\mathrm{C} 51 \mathrm{~A}-\mathrm{C} 50 \mathrm{~A}-\mathrm{C} 49 \mathrm{~A}$ & $108.7(8)$ \\
\hline $\mathrm{C} 14-\mathrm{C} 15-\mathrm{H} 15 \mathrm{~A}$ & 109.7 & $\mathrm{C} 51 \mathrm{~A}-\mathrm{C} 50 \mathrm{~A}-\mathrm{H} 50 \mathrm{~A}$ & 110.0 \\
\hline $\mathrm{C} 16-\mathrm{C} 15-\mathrm{H} 15 \mathrm{~B}$ & 109.7 & $\mathrm{C} 49 \mathrm{~A}-\mathrm{C} 50 \mathrm{~A}-\mathrm{H} 50 \mathrm{~A}$ & 110.0 \\
\hline $\mathrm{C} 14-\mathrm{C} 15-\mathrm{H} 15 \mathrm{~B}$ & 109.7 & $\mathrm{C} 51 \mathrm{~A}-\mathrm{C} 50 \mathrm{~A}-\mathrm{H} 50 \mathrm{~B}$ & 110.0 \\
\hline $\mathrm{H} 15 \mathrm{~A}-\mathrm{C} 15-\mathrm{H} 15 \mathrm{~B}$ & 108.2 & $\mathrm{C} 49 \mathrm{~A}-\mathrm{C} 50 \mathrm{~A}-\mathrm{H} 50 \mathrm{~B}$ & 110.0 \\
\hline $\mathrm{C} 17-\mathrm{C} 16-\mathrm{C} 15$ & $111.4(4)$ & $\mathrm{H} 50 \mathrm{~A}-\mathrm{C} 50 \mathrm{~A}-\mathrm{H} 50 \mathrm{~B}$ & 108.3 \\
\hline $\mathrm{C} 17-\mathrm{C} 16-\mathrm{H} 16 \mathrm{~A}$ & 109.4 & $\mathrm{O} 1 \mathrm{~A}-\mathrm{C} 51 \mathrm{~A}-\mathrm{C} 50 \mathrm{~A}$ & $105.6(7)$ \\
\hline $\mathrm{C} 15-\mathrm{C} 16-\mathrm{H} 16 \mathrm{~A}$ & 109.4 & $\mathrm{O} 1 \mathrm{~A}-\mathrm{C} 51 \mathrm{~A}-\mathrm{H} 51 \mathrm{~A}$ & 110.6 \\
\hline $\mathrm{C} 17-\mathrm{C} 16-\mathrm{H} 16 \mathrm{~B}$ & 109.4 & $\mathrm{C} 50 \mathrm{~A}-\mathrm{C} 51 \mathrm{~A}-\mathrm{H} 51 \mathrm{~A}$ & 110.6 \\
\hline $\mathrm{C} 15-\mathrm{C} 16-\mathrm{H} 16 \mathrm{~B}$ & 109.4 & $\mathrm{O} 1 \mathrm{~A}-\mathrm{C} 51 \mathrm{~A}-\mathrm{H} 51 \mathrm{~B}$ & 110.6 \\
\hline $\mathrm{H} 16 \mathrm{~A}-\mathrm{C} 16-\mathrm{H} 16 \mathrm{~B}$ & 108.0 & $\mathrm{C} 50 \mathrm{~A}-\mathrm{C} 51 \mathrm{~A}-\mathrm{H} 51 \mathrm{~B}$ & 110.6 \\
\hline $\mathrm{C} 16-\mathrm{C} 17-\mathrm{C} 18$ & $111.3(4)$ & $\mathrm{H} 51 \mathrm{~A}-\mathrm{C} 51 \mathrm{~A}-\mathrm{H} 51 \mathrm{~B}$ & 108.7 \\
\hline $\mathrm{C} 16-\mathrm{C} 17-\mathrm{H} 17 \mathrm{~A}$ & 109.4 & $\mathrm{C} 51 \mathrm{~B}-\mathrm{O} 1 \mathrm{~B}-\mathrm{C} 48 \mathrm{~B}$ & $109.9(12)$ \\
\hline $\mathrm{C} 18-\mathrm{C} 17-\mathrm{H} 17 \mathrm{~A}$ & 109.4 & $\mathrm{C} 51 \mathrm{~B}-\mathrm{O} 1 \mathrm{~B}-\mathrm{LI}$ & $116.5(12)$ \\
\hline $\mathrm{C} 16-\mathrm{C} 17-\mathrm{H} 17 \mathrm{~B}$ & 109.4 & $\mathrm{C} 48 \mathrm{~B}-\mathrm{O} 1 \mathrm{~B}-\mathrm{LI}$ & $125.7(14)$ \\
\hline $\mathrm{C} 18-\mathrm{C} 17-\mathrm{H} 17 \mathrm{~B}$ & 109.4 & $\mathrm{O} 1 \mathrm{~B}-\mathrm{C} 48 \mathrm{~B}-\mathrm{C} 49 \mathrm{~B}$ & $102.4(12)$ \\
\hline $\mathrm{H} 17 \mathrm{~A}-\mathrm{C} 17-\mathrm{H} 17 \mathrm{~B}$ & 108.0 & $\mathrm{O} 1 \mathrm{~B}-\mathrm{C} 48 \mathrm{~B}-\mathrm{H} 48 \mathrm{C}$ & 111.3 \\
\hline $\mathrm{C} 13-\mathrm{C} 18-\mathrm{C} 17$ & $111.0(4)$ & $\mathrm{C} 49 \mathrm{~B}-\mathrm{C} 48 \mathrm{~B}-\mathrm{H} 48 \mathrm{C}$ & 111.3 \\
\hline $\mathrm{C} 13-\mathrm{C} 18-\mathrm{H} 18 \mathrm{~A}$ & 109.4 & $\mathrm{O} 1 \mathrm{~B}-\mathrm{C} 48 \mathrm{~B}-\mathrm{H} 48 \mathrm{D}$ & 111.3 \\
\hline $\mathrm{C} 17-\mathrm{C} 18-\mathrm{H} 18 \mathrm{~A}$ & 109.4 & $\mathrm{C} 49 \mathrm{~B}-\mathrm{C} 48 \mathrm{~B}-\mathrm{H} 48 \mathrm{D}$ & 111.3 \\
\hline $\mathrm{C} 13-\mathrm{C} 18-\mathrm{H} 18 \mathrm{~B}$ & 109.4 & $\mathrm{H} 48 \mathrm{C}-\mathrm{C} 48 \mathrm{~B}-\mathrm{H} 48 \mathrm{D}$ & 109.2 \\
\hline $\mathrm{C} 17-\mathrm{C} 18-\mathrm{H} 18 \mathrm{~B}$ & 109.4 & $\mathrm{C} 48 \mathrm{~B}-\mathrm{C} 49 \mathrm{~B}-\mathrm{C} 50 \mathrm{~B}$ & $103.2(13)$ \\
\hline
\end{tabular}




\begin{tabular}{|c|c|}
\hline $\mathrm{H} 18 \mathrm{~A}-\mathrm{C} 18-\mathrm{H} 18 \mathrm{~B}$ & 108.0 \\
\hline $\mathrm{N} 3-\mathrm{C} 19 \mathrm{~A}-\mathrm{C} 24 \mathrm{~A}$ & $112.4(5)$ \\
\hline $\mathrm{N} 3-\mathrm{C} 19 \mathrm{~A}-\mathrm{C} 20 \mathrm{~A}$ & $111.6(5)$ \\
\hline $\mathrm{C} 24 \mathrm{~A}-\mathrm{C} 19 \mathrm{~A}-\mathrm{C} 20 \mathrm{~A}$ & $111.1(5)$ \\
\hline N3-C19A-H19A & 107.2 \\
\hline $\mathrm{C} 24 \mathrm{~A}-\mathrm{C} 19 \mathrm{~A}-\mathrm{H} 19 \mathrm{~A}$ & 107.2 \\
\hline $\mathrm{C} 20 \mathrm{~A}-\mathrm{C} 19 \mathrm{~A}-\mathrm{H} 19 \mathrm{~A}$ & 107.2 \\
\hline $\mathrm{C} 19 \mathrm{~A}-\mathrm{C} 20 \mathrm{~A}-\mathrm{C} 21 \mathrm{~A}$ & $109.9(5)$ \\
\hline $\mathrm{C} 19 \mathrm{~A}-\mathrm{C} 20 \mathrm{~A}-\mathrm{H} 20 \mathrm{~A}$ & 109.7 \\
\hline $\mathrm{C} 21 \mathrm{~A}-\mathrm{C} 20 \mathrm{~A}-\mathrm{H} 20 \mathrm{~A}$ & 109.7 \\
\hline $\mathrm{C} 19 \mathrm{~A}-\mathrm{C} 20 \mathrm{~A}-\mathrm{H} 20 \mathrm{~B}$ & 109.7 \\
\hline $\mathrm{C} 21 \mathrm{~A}-\mathrm{C} 20 \mathrm{~A}-\mathrm{H} 20 \mathrm{~B}$ & 109.7 \\
\hline $\mathrm{H} 20 \mathrm{~A}-\mathrm{C} 20 \mathrm{~A}-\mathrm{H} 20 \mathrm{~B}$ & 108.2 \\
\hline $\mathrm{C} 22 \mathrm{~A}-\mathrm{C} 21 \mathrm{~A}-\mathrm{C} 20 \mathrm{~A}$ & $111.4(6)$ \\
\hline $\mathrm{C} 22 \mathrm{~A}-\mathrm{C} 21 \mathrm{~A}-\mathrm{H} 21 \mathrm{~A}$ & 109.3 \\
\hline $\mathrm{C} 20 \mathrm{~A}-\mathrm{C} 21 \mathrm{~A}-\mathrm{H} 21 \mathrm{~A}$ & 109.3 \\
\hline $\mathrm{C} 22 \mathrm{~A}-\mathrm{C} 21 \mathrm{~A}-\mathrm{H} 21 \mathrm{~B}$ & 109.3 \\
\hline $\mathrm{C} 20 \mathrm{~A}-\mathrm{C} 21 \mathrm{~A}-\mathrm{H} 21 \mathrm{~B}$ & 109.3 \\
\hline $\mathrm{H} 21 \mathrm{~A}-\mathrm{C} 21 \mathrm{~A}-\mathrm{H} 21 \mathrm{~B}$ & 108.0 \\
\hline $\mathrm{C} 23 \mathrm{~A}-\mathrm{C} 22 \mathrm{~A}-\mathrm{C} 21 \mathrm{~A}$ & $112.3(6)$ \\
\hline $\mathrm{C} 23 \mathrm{~A}-\mathrm{C} 22 \mathrm{~A}-\mathrm{H} 22 \mathrm{~A}$ & 109.1 \\
\hline $\mathrm{C} 21 \mathrm{~A}-\mathrm{C} 22 \mathrm{~A}-\mathrm{H} 22 \mathrm{~A}$ & 109.1 \\
\hline $\mathrm{C} 23 \mathrm{~A}-\mathrm{C} 22 \mathrm{~A}-\mathrm{H} 22 \mathrm{~B}$ & 109.1 \\
\hline $\mathrm{C} 21 \mathrm{~A}-\mathrm{C} 22 \mathrm{~A}-\mathrm{H} 22 \mathrm{~B}$ & 109.1 \\
\hline $\mathrm{H} 22 \mathrm{~A}-\mathrm{C} 22 \mathrm{~A}-\mathrm{H} 22 \mathrm{~B}$ & 107.9 \\
\hline $\mathrm{C} 22 \mathrm{~A}-\mathrm{C} 23 \mathrm{~A}-\mathrm{C} 24 \mathrm{~A}$ & $112.3(6)$ \\
\hline $\mathrm{C} 22 \mathrm{~A}-\mathrm{C} 23 \mathrm{~A}-\mathrm{H} 23 \mathrm{~A}$ & 109.1 \\
\hline $\mathrm{C} 24 \mathrm{~A}-\mathrm{C} 23 \mathrm{~A}-\mathrm{H} 23 \mathrm{~A}$ & 109.1 \\
\hline $\mathrm{C} 22 \mathrm{~A}-\mathrm{C} 23 \mathrm{~A}-\mathrm{H} 23 \mathrm{~B}$ & 109.1 \\
\hline $\mathrm{C} 24 \mathrm{~A}-\mathrm{C} 23 \mathrm{~A}-\mathrm{H} 23 \mathrm{~B}$ & 109.1 \\
\hline $\mathrm{H} 23 \mathrm{~A}-\mathrm{C} 23 \mathrm{~A}-\mathrm{H} 23 \mathrm{~B}$ & 107.9 \\
\hline $\mathrm{C} 19 \mathrm{~A}-\mathrm{C} 24 \mathrm{~A}-\mathrm{C} 23 \mathrm{~A}$ & $110.1(5)$ \\
\hline $\mathrm{C} 19 \mathrm{~A}-\mathrm{C} 24 \mathrm{~A}-\mathrm{H} 24 \mathrm{~A}$ & 109.6 \\
\hline $\mathrm{C} 23 \mathrm{~A}-\mathrm{C} 24 \mathrm{~A}-\mathrm{H} 24 \mathrm{~A}$ & 109.6 \\
\hline $\mathrm{C} 19 \mathrm{~A}-\mathrm{C} 24 \mathrm{~A}-\mathrm{H} 24 \mathrm{~B}$ & 109.6 \\
\hline $\mathrm{C} 23 \mathrm{~A}-\mathrm{C} 24 \mathrm{~A}-\mathrm{H} 24 \mathrm{~B}$ & 109.6 \\
\hline $\mathrm{H} 24 \mathrm{~A}-\mathrm{C} 24 \mathrm{~A}-\mathrm{H} 24 \mathrm{~B}$ & 108.2 \\
\hline N3-C19B-C24B & $112.2(15)$ \\
\hline N3-C19B-C20B & $110.5(15)$ \\
\hline $\mathrm{C} 24 \mathrm{~B}-\mathrm{C} 19 \mathrm{~B}-\mathrm{C} 20 \mathrm{~B}$ & $111.1(15)$ \\
\hline N3-C19B-H19B & 107.6 \\
\hline $\mathrm{C} 24 \mathrm{~B}-\mathrm{C} 19 \mathrm{~B}-\mathrm{H} 19 \mathrm{~B}$ & 107.6 \\
\hline $\mathrm{C} 20 \mathrm{~B}-\mathrm{C} 19 \mathrm{~B}-\mathrm{H} 19 \mathrm{~B}$ & 107.6 \\
\hline $\mathrm{C} 19 \mathrm{~B}-\mathrm{C} 20 \mathrm{~B}-\mathrm{C} 21 \mathrm{~B}$ & $110.2(16)$ \\
\hline $\mathrm{C} 19 \mathrm{~B}-\mathrm{C} 20 \mathrm{~B}-\mathrm{H} 20 \mathrm{C}$ & 109.6 \\
\hline $\mathrm{C} 21 \mathrm{~B}-\mathrm{C} 20 \mathrm{~B}-\mathrm{H} 20 \mathrm{C}$ & 109.6 \\
\hline $\mathrm{C} 19 \mathrm{~B}-\mathrm{C} 20 \mathrm{~B}-\mathrm{H} 20 \mathrm{D}$ & 109.6 \\
\hline & \\
\hline
\end{tabular}

$\begin{array}{ll}\text { C48B-C49B-H49C } & 111.1 \\ \text { C50B-C49B-H49C } & 111.1 \\ \text { C48B-C49B-H49D } & 111.1 \\ \text { C50B-C49B-H49D } & 111.1 \\ \text { H49C-C49B-H49D } & 109.1 \\ \text { C51B-C50B-C49B } & 99.5(13) \\ \text { C51B-C50B-H50C } & 111.9 \\ \text { C49B-C50B-H50C } & 111.9 \\ \text { C51B-C50B-H50D } & 111.9 \\ \text { C49B-C50B-H50D } & 111.9 \\ \text { H50C-C50B-H50D } & 109.6 \\ \text { O1B-C51B-C50B } & 104.8(12) \\ \text { O1B-C51B-H51C } & 110.8 \\ \text { C50B-C51B-H51C } & 110.8 \\ \text { O1B-C51B-H51D } & 110.8 \\ \text { C50B-C51B-H51D } & 110.8 \\ \text { H51C-C51B-H51D } & 108.9 \\ \text { C55A-O2A-C52A } & 110.0(7) \\ \text { C55A-O2A-LI } & 124.7(7) \\ \text { C52A-O2A-LI } & 125.3(9) \\ \text { O2A-C52A-C53A } & 105.7(8) \\ \text { O2A-C52A-H52A } & 110.6 \\ \text { C53A-C52A-H52A } & 110.6 \\ \text { O2A-C52A-H52B } & 110.6 \\ \text { C53A-C52A-H52B } & 110.6 \\ \text { H52A-C52A-H52B } & 108.7 \\ \text { C54A-C53A-C52A } & 107.0(7) \\ \text { C54A-C53A-H53A } & 110.3 \\ \text { C52A-C53A-H53A } & 110.3 \\ \text { C54A-C53A-H53B } & 110.3 \\ \text { C52A-C53A-H53B } & 110.3 \\ \text { H53A-C53A-H53B } & 108.6 \\ \text { C55A-C54A-C53A } & 108.6(7) \\ \text { C55A-C54A-H54A } & 110.0 \\ \text { C53A-C54A-H54A } & 110.0 \\ \text { C55A-C54A-H54B } & 110.0 \\ \text { C53A-C54A-H54B } & 110.0 \\ \text { H54A-C54A-H54B } & 108.4 \\ \text { O2A-C55A-C54A } & 106.9(7) \\ \text { O2A-C55A-H55A } & 110.3 \\ \text { C54A-C55A-H55A } & 110.3 \\ \text { O2A-C55A-H55B } & 110.3 \\ \text { C54A-C55A-H55B } & 110.3 \\ \text { H55A-C55A-H55B } & 108.6 \\ \text { C55B-O2B-C52B } & 110.4(17) \\ \text { C55B-O2B-LI } & 124(3) \\ \text { C52B-O2B-LI } & 103.3(17) \\ \text { O2B-C52B-C53B } & \\ & \end{array}$




\begin{tabular}{|c|c|}
\hline $\mathrm{H} 20 \mathrm{C}-\mathrm{C} 20 \mathrm{~B}-\mathrm{H} 20 \mathrm{D}$ & 108.1 \\
\hline $\mathrm{C} 22 \mathrm{~B}-\mathrm{C} 21 \mathrm{~B}-\mathrm{C} 20 \mathrm{~B}$ & $110.7(16)$ \\
\hline $\mathrm{C} 22 \mathrm{~B}-\mathrm{C} 21 \mathrm{~B}-\mathrm{H} 21 \mathrm{C}$ & 109.5 \\
\hline $\mathrm{C} 20 \mathrm{~B}-\mathrm{C} 21 \mathrm{~B}-\mathrm{H} 21 \mathrm{C}$ & 109.5 \\
\hline $\mathrm{C} 22 \mathrm{~B}-\mathrm{C} 21 \mathrm{~B}-\mathrm{H} 21 \mathrm{D}$ & 109.5 \\
\hline $\mathrm{C} 20 \mathrm{~B}-\mathrm{C} 21 \mathrm{~B}-\mathrm{H} 21 \mathrm{D}$ & 109.5 \\
\hline $\mathrm{H} 21 \mathrm{C}-\mathrm{C} 21 \mathrm{~B}-\mathrm{H} 21 \mathrm{D}$ & 108.1 \\
\hline $\mathrm{C} 23 \mathrm{~B}-\mathrm{C} 22 \mathrm{~B}-\mathrm{C} 21 \mathrm{~B}$ & $110.3(16)$ \\
\hline $\mathrm{C} 23 \mathrm{~B}-\mathrm{C} 22 \mathrm{~B}-\mathrm{H} 22 \mathrm{C}$ & 109.6 \\
\hline $\mathrm{C} 21 \mathrm{~B}-\mathrm{C} 22 \mathrm{~B}-\mathrm{H} 22 \mathrm{C}$ & 109.6 \\
\hline $\mathrm{C} 23 \mathrm{~B}-\mathrm{C} 22 \mathrm{~B}-\mathrm{H} 22 \mathrm{D}$ & 109.6 \\
\hline $\mathrm{C} 21 \mathrm{~B}-\mathrm{C} 22 \mathrm{~B}-\mathrm{H} 22 \mathrm{D}$ & 109.6 \\
\hline $\mathrm{H} 22 \mathrm{C}-\mathrm{C} 22 \mathrm{~B}-\mathrm{H} 22 \mathrm{D}$ & 108.1 \\
\hline $\mathrm{C} 22 \mathrm{~B}-\mathrm{C} 23 \mathrm{~B}-\mathrm{C} 24 \mathrm{~B}$ & $110.8(16)$ \\
\hline $\mathrm{C} 22 \mathrm{~B}-\mathrm{C} 23 \mathrm{~B}-\mathrm{H} 23 \mathrm{C}$ & 109.5 \\
\hline $\mathrm{C} 24 \mathrm{~B}-\mathrm{C} 23 \mathrm{~B}-\mathrm{H} 23 \mathrm{C}$ & 109.5 \\
\hline $\mathrm{C} 22 \mathrm{~B}-\mathrm{C} 23 \mathrm{~B}-\mathrm{H} 23 \mathrm{D}$ & 109.5 \\
\hline $\mathrm{C} 24 \mathrm{~B}-\mathrm{C} 23 \mathrm{~B}-\mathrm{H} 23 \mathrm{D}$ & 109.5 \\
\hline $\mathrm{H} 23 \mathrm{C}-\mathrm{C} 23 \mathrm{~B}-\mathrm{H} 23 \mathrm{D}$ & 108.1 \\
\hline $\mathrm{C} 19 \mathrm{~B}-\mathrm{C} 24 \mathrm{~B}-\mathrm{C} 23 \mathrm{~B}$ & $108.8(15)$ \\
\hline $\mathrm{C} 19 \mathrm{~B}-\mathrm{C} 24 \mathrm{~B}-\mathrm{H} 24 \mathrm{C}$ & 109.9 \\
\hline $\mathrm{C} 23 \mathrm{~B}-\mathrm{C} 24 \mathrm{~B}-\mathrm{H} 24 \mathrm{C}$ & 109.9 \\
\hline $\mathrm{C} 19 \mathrm{~B}-\mathrm{C} 24 \mathrm{~B}-\mathrm{H} 24 \mathrm{D}$ & 109.9 \\
\hline $\mathrm{C} 7-\mathrm{N} 1-\mathrm{C} 1-\mathrm{N} 2$ & $-166.2(3)$ \\
\hline $\mathrm{HO}-\mathrm{N} 1-\mathrm{C} 1-\mathrm{N} 2$ & $-6.4(3)$ \\
\hline $\mathrm{C} 7-\mathrm{N} 1-\mathrm{C} 1-\mathrm{N} 3$ & $15.7(6)$ \\
\hline $\mathrm{HO}-\mathrm{N} 1-\mathrm{C} 1-\mathrm{N} 3$ & $175.4(3)$ \\
\hline $\mathrm{C} 7-\mathrm{N} 1-\mathrm{C} 1-\mathrm{HO}$ & $-159.8(4)$ \\
\hline $\mathrm{C} 13-\mathrm{N} 2-\mathrm{C} 1-\mathrm{N} 1$ & $-170.7(3)$ \\
\hline $\mathrm{HO}-\mathrm{N} 2-\mathrm{C} 1-\mathrm{N} 1$ & $6.4(3)$ \\
\hline $\mathrm{C} 13-\mathrm{N} 2-\mathrm{C} 1-\mathrm{N} 3$ & $7.5(6)$ \\
\hline $\mathrm{HO}-\mathrm{N} 2-\mathrm{C} 1-\mathrm{N} 3$ & $-175.4(3)$ \\
\hline $\mathrm{C} 13-\mathrm{N} 2-\mathrm{C} 1-\mathrm{HO}$ & $-177.1(4)$ \\
\hline $\mathrm{C} 2-\mathrm{N} 3-\mathrm{C} 1-\mathrm{N} 1$ & $-124.8(4)$ \\
\hline $\mathrm{C} 19 \mathrm{~B}-\mathrm{N} 3-\mathrm{C} 1-\mathrm{N} 1$ & $65.9(9)$ \\
\hline $\mathrm{C} 19 \mathrm{~A}-\mathrm{N} 3-\mathrm{C} 1-\mathrm{N} 1$ & $86.9(5)$ \\
\hline $\mathrm{C} 2-\mathrm{N} 3-\mathrm{C} 1-\mathrm{N} 2$ & $57.1(5)$ \\
\hline $\mathrm{C} 19 \mathrm{~B}-\mathrm{N} 3-\mathrm{C} 1-\mathrm{N} 2$ & $-112.1(8)$ \\
\hline $\mathrm{C} 19 \mathrm{~A}-\mathrm{N} 3-\mathrm{C} 1-\mathrm{N} 2$ & $-91.1(5)$ \\
\hline $\mathrm{C} 25-\mathrm{N} 4-\mathrm{C} 2-\mathrm{N} 3$ & $-176.7(4)$ \\
\hline $\mathrm{C} 25-\mathrm{N} 4-\mathrm{C} 2-\mathrm{C} 3$ & $-2.1(7)$ \\
\hline $\mathrm{C} 1-\mathrm{N} 3-\mathrm{C} 2-\mathrm{N} 4$ & $-148.1(4)$ \\
\hline $\mathrm{C} 19 \mathrm{~B}-\mathrm{N} 3-\mathrm{C} 2-\mathrm{N} 4$ & $18.0(11)$ \\
\hline $\mathrm{C} 19 \mathrm{~A}-\mathrm{N} 3-\mathrm{C} 2-\mathrm{N} 4$ & $1.7(6)$ \\
\hline $\mathrm{C} 1-\mathrm{N} 3-\mathrm{C} 2-\mathrm{C} 3$ & $36.6(5)$ \\
\hline $\mathrm{C} 19 \mathrm{~B}-\mathrm{N} 3-\mathrm{C} 2-\mathrm{C} 3$ & $-157.3(10)$ \\
\hline $\mathrm{C} 19 \mathrm{~A}-\mathrm{N} 3-\mathrm{C} 2-\mathrm{C} 3$ & $-173.7(4)$ \\
\hline
\end{tabular}

$\begin{array}{ll}\mathrm{O} 2 \mathrm{~B}-\mathrm{C} 52 \mathrm{~B}-\mathrm{H} 52 \mathrm{C} & 111.1 \\ \mathrm{C} 53 \mathrm{~B}-\mathrm{C} 52 \mathrm{~B}-\mathrm{H} 52 \mathrm{C} & 111.1 \\ \text { O2B-C52B-H52D } & 111.1 \\ \mathrm{C} 53 \mathrm{~B}-\mathrm{C} 52 \mathrm{~B}-\mathrm{H} 52 \mathrm{D} & 111.1 \\ \mathrm{H} 52 \mathrm{C}-\mathrm{C} 52 \mathrm{~B}-\mathrm{H} 52 \mathrm{D} & 109.1 \\ \mathrm{C} 54 \mathrm{~B}-\mathrm{C} 53 \mathrm{~B}-\mathrm{C} 52 \mathrm{~B} & 107.6(17) \\ \mathrm{C} 54 \mathrm{~B}-\mathrm{C} 53 \mathrm{~B}-\mathrm{H} 53 \mathrm{C} & 110.2 \\ \mathrm{C} 52 \mathrm{~B}-\mathrm{C} 53 \mathrm{~B}-\mathrm{H} 53 \mathrm{C} & 110.2 \\ \mathrm{C} 54 \mathrm{~B}-\mathrm{C} 53 \mathrm{~B}-\mathrm{H} 53 \mathrm{D} & 110.2 \\ \mathrm{C} 52 \mathrm{~B}-\mathrm{C} 53 \mathrm{~B}-\mathrm{H} 53 \mathrm{D} & 110.2 \\ \mathrm{H} 53 \mathrm{C}-\mathrm{C} 53 \mathrm{~B}-\mathrm{H} 53 \mathrm{D} & 108.5 \\ \mathrm{C} 53 \mathrm{~B}-\mathrm{C} 54 \mathrm{~B}-\mathrm{C} 55 \mathrm{~B} & 107.3(16) \\ \mathrm{C} 53 \mathrm{~B}-\mathrm{C} 54 \mathrm{~B}-\mathrm{H} 54 \mathrm{C} & 110.3 \\ \mathrm{C} 55 \mathrm{~B}-\mathrm{C} 54 \mathrm{~B}-\mathrm{H} 54 \mathrm{C} & 110.3 \\ \text { C53B-C54B-H54D } & 110.3 \\ \text { C55B-C54B-H54D } & 110.3 \\ \text { H54C-C54B-H54D } & 108.5 \\ \text { O2B-C55B-C54B } & 106.8(16) \\ \text { O2B-C55B-H55C } & 110.4 \\ \text { C54B-C55B-H55C } & 110.4 \\ \text { O2B-C55B-H55D } & 110.4 \\ \text { C54B-C55B-H55D } & 110.4 \\ \text { H55C-C55B-H55D } & 108.6\end{array}$

$\mathrm{N} 4-\mathrm{C} 25-\mathrm{C} 30-\mathrm{C} 29 \quad-63.1(5)$

$\mathrm{C} 26-\mathrm{C} 25-\mathrm{C} 30-\mathrm{C} 29 \quad 55.9$ (6)

$\mathrm{C} 42-\mathrm{N} 6-\mathrm{C} 31-\mathrm{N} 5 \quad-174.3(4)$

$\mathrm{HO}-\mathrm{N} 6-\mathrm{C} 31-\mathrm{N} 5 \quad-9.5(4)$

$\mathrm{C} 42-\mathrm{N} 6-\mathrm{C} 31-\mathrm{C} 32$

$\mathrm{HO}-\mathrm{N} 6-\mathrm{C} 31-\mathrm{C} 32$

$\mathrm{C} 42-\mathrm{N} 6-\mathrm{C} 31-\mathrm{HO} \quad-164.8(4)$

$\mathrm{C} 36-\mathrm{N} 5-\mathrm{C} 31-\mathrm{N} 6 \quad-175.4(4)$

$\mathrm{HO}-\mathrm{N} 5-\mathrm{C} 31-\mathrm{N} 6 \quad 9.6(4)$

$\mathrm{C} 36-\mathrm{N} 5-\mathrm{C} 31-\mathrm{C} 32$

$\mathrm{HO}-\mathrm{N} 5-\mathrm{C} 31-\mathrm{C} 32 \quad-171.1(4)$

$\mathrm{C} 36-\mathrm{N} 5-\mathrm{C} 31-\mathrm{HO} \quad 175.1$ (4)

$\mathrm{N} 6-\mathrm{C} 31-\mathrm{C} 32-\mathrm{C} 33 \quad 87.0(5)$

$\mathrm{N} 5-\mathrm{C} 31-\mathrm{C} 32-\mathrm{C} 33 \quad-92.3(5)$

$\mathrm{C} 31-\mathrm{C} 32-\mathrm{C} 33-\mathrm{C} 34 \quad 177.6$ (4)

$\mathrm{C} 32-\mathrm{C} 33-\mathrm{C} 34-\mathrm{C} 35 \quad 65.5(6)$

$\mathrm{C} 31-\mathrm{N} 5-\mathrm{C} 36-\mathrm{C} 37 \quad 103.3(5)$

$\mathrm{HO}-\mathrm{N} 5-\mathrm{C} 36-\mathrm{C} 37 \quad-84.7(5)$

$\mathrm{C} 31-\mathrm{N} 5-\mathrm{C} 36-\mathrm{C} 41 \quad-135.2(4)$

$\mathrm{HO}-\mathrm{N} 5-\mathrm{C} 36-\mathrm{C} 41 \quad 36.8(6)$

$\mathrm{N} 5-\mathrm{C} 36-\mathrm{C} 37-\mathrm{C} 38 \quad 177.1(4)$

$\mathrm{C} 41-\mathrm{C} 36-\mathrm{C} 37-\mathrm{C} 38 \quad 55.6(6)$

$\mathrm{C} 36-\mathrm{C} 37-\mathrm{C} 38-\mathrm{C} 39 \quad-56.1(7)$

$\mathrm{C} 37-\mathrm{C} 38-\mathrm{C} 39-\mathrm{C} 40 \quad 54.4(7)$ 


$\begin{array}{ll}\text { N4-C2-C3-C } 4 & -85.4(5) \\ \text { N3-C2-C3-C4 } & 89.3(4) \\ \text { C2-C3-C4-C5 } & -172.0(4) \\ \text { C3-C4-C5-C6 } & 176.4(4) \\ \text { C1-N1-C7-C12 } & 107.3(4) \\ \text { HO-N1-C7-C12 } & -40.2(6) \\ \text { C1-N1-C7-C } & -131.5(4) \\ \text { HO-N1-C7-C } & 80.9(5) \\ \text { N1-C7-C8-C9 } & -179.8(4) \\ \text { C12-C7-C8-C9 } & -57.5(5) \\ \text { C7-C8-C9-C10 } & 56.2(6) \\ \text { C8-C9-C10-C11 } & -54.3(6) \\ \text { C9-C10-C11-C12 } & 54.6(6) \\ \text { N1-C7-C12-C11 } & -179.3(4) \\ \text { C8-C7-C12-C11 } & 58.7(5) \\ \text { C10-C11-C12-C7 } & -57.6(5) \\ \text { C1-N2-C13-C14 } & -116.1(4) \\ \text { HO-N2-C13-C14 } & 68.6(5) \\ \text { C1-N2-C13-C18 } & 124.1(4) \\ \text { HO-N2-C13-C18 } & -51.1(6) \\ \text { N2-C13-C14-C15 } & -177.3(4) \\ \text { C18-C13-C14-C15 } & -57.9(5) \\ \text { C13-C14-C15-C16 } & 57.4(6) \\ \text { C14-C15-C16-C17 } & -55.8(6) \\ \text { C15-C16-C17-C18 } & 56.2(6) \\ \text { N2-C13-C18-C17 } & 177.6(4) \\ \text { C14-C13-C18-C17 } & 57.1(5) \\ \text { C16-C17-C18-C13 } & -56.8(6) \\ \text { C2-N3-C19A-C24A } & -159.8(5) \\ \text { C1-N3-C19A-C24A } & -9.8(7) \\ \text { C2-N3-C19A-C20A } & 74.6(6) \\ \text { C1-N3-C19A-C20A } & -135.4(5) \\ \text { N3-C19A-C20A-C21A } & -175.1(6) \\ \text { C24A-C19A-C20A-C21A } & 58.7(8) \\ \text { C19A-C20A-C21A-C22A } & -55.8(8) \\ \text { C20A-C21A-C22A-C23A } & 53.4(8) \\ \text { C21A-C22A-C23A-C24A } & -52.7(8) \\ \text { N3-C19A-C24A-C23A } & 176.5(5) \\ \text { C20A-C19A-C24A-C23A } & -57.7(7) \\ \text { C22A-C23A-C24A-C19A } & 54.5(8) \\ \text { C2-N3-C19B-C24B } & -76.8(18) \\ \text { C1-N3-C19B-C24B } & 90.7(16) \\ \text { C2-N3-C19B-C20B } & 48(2) \\ \text { C1-N3-C19B-C20B } & -144.7(15) \\ \text { N3-C19B-C20B-C21B } & 176.9(17) \\ \text { C24B-C19B-C20B-C21B } & -58(3) \\ \text { C19B-C20B-C21B-C22B } & 57(3) \\ \text { C20B-C21B-C22B-C23B } & -58(3) \\ & \end{array}$

$\begin{array}{ll}\text { C38-C39-C40-C41 } & -55.2(7) \\ \text { N5-C36-C41-C40 } & -176.1(4) \\ \text { C37-C36-C41-C40 } & -53.9(6) \\ \text { C39-C40-C41-C36 } & 55.2(6) \\ \text { C31-N6-C42-C47 } & 92.4(5) \\ \text { HO-N6-C42-C47 } & -63.4(6) \\ \text { C31-N6-C42-C43 } & -145.0(4) \\ \text { HO-N6-C42-C43 } & 59.3(6) \\ \text { N6-C42-C43-C44 } & -176.4(3) \\ \text { C47-C42-C43-C44 } & -54.1(5) \\ \text { C42-C43-C44-C45 } & 55.8(5) \\ \text { C43-C44-C45-C46 } & -56.3(5) \\ \text { C44-C45-C46-C47 } & 55.8(5) \\ \text { N6-C42-C47-C46 } & 175.1(4) \\ \text { C43-C42-C47-C46 } & 53.8(5) \\ \text { C45-C46-C47-C42 } & -54.7(5) \\ \text { C51A-O1A-C48A-C49A } & -18.8(13) \\ \text { LI-O1A-C48A-C49A } & 144.5(9) \\ \text { O1A-C48A-C49A-C50A } & 23.9(15) \\ \text { C48A-C49A-C50A-C51A } & -21.4(15) \\ \text { C48A-O1A-C51A-C50A } & 5.8(12) \\ \text { LI-O1A-C51A-C50A } & -157.6(11) \\ \text { C49A-C50A-C51A-O1A } & 9.7(14) \\ \text { O2B-LI-O1B-C51B } & 75(2) \\ \text { CL2-LI-O1B-C51B } & -164.7(12) \\ \text { CL1-LI-O1B-C51B } & -47.2(15) \\ \text { HO-LI-O1B-C51B } & -105.5(14) \\ \text { O2B-LI-O1B-C48B } & -140(3) \\ \text { CL2-LI-O1B-C48B } & -19(2) \\ \text { CL1-LI-O1B-C48B } & 99(2) \\ \text { HO-LI-O1B-C48B } & 40(2) \\ \text { C51B-O1B-C48B-C49B } & -8(3) \\ \text { LI-O1B-C48B-C49B } & -155.8(17) \\ \text { O1B-C48B-C49B-C50B } & 33(3) \\ \text { C48B-C49B-C50B-C51B } & -44(2) \\ \text { C48B-O1B-C51B-C50B } & -20(2) \\ \text { LI-O1B-C51B-C50B } & 131.1(18) \\ \text { C49B-C50B-C51B-O1B } & 39(2) \\ \text { C55A-O2A-C52A-C53A } & 12.7(17) \\ \text { LI-O2A-C52A-C53A } & -168.1(13) \\ \text { O2A-C52A-C53A-C54A } & -6.5(16) \\ \text { C52A-C53A-C54A-C55A } & -1.6(14) \\ \text { C52A-O2A-C55A-C54A } & -13.8(16) \\ \text { LI-O2A-C55A-C54A } & 167.0(11) \\ \text { C53A-C54A-C55A-O2A } & 9.4(14) \\ \text { O1B-LI-O2B-C55B } & 99(7) \\ \text { CL2-LI-O2B-C55B } & -25(9) \\ \text { CL1-LI-O2B-C55B } & -138(7) \\ & \end{array}$




$\begin{array}{llll}\mathrm{C} 21 \mathrm{~B}-\mathrm{C} 22 \mathrm{~B}-\mathrm{C} 23 \mathrm{~B}-\mathrm{C} 24 \mathrm{~B} & 59(3) & \mathrm{HO}-\mathrm{L}-\mathrm{O} 2 \mathrm{~B}-\mathrm{C} 55 \mathrm{~B} & -81(8) \\ \mathrm{N} 3-\mathrm{C} 19 \mathrm{~B}-\mathrm{C} 24 \mathrm{~B}-\mathrm{C} 23 \mathrm{~B} & -177.8(16) & \mathrm{O} 1 \mathrm{~B}-\mathrm{LI}-\mathrm{O} 2 \mathrm{~B}-\mathrm{C} 52 \mathrm{~B} & -63(7) \\ \mathrm{C} 20 \mathrm{~B}-\mathrm{C} 19 \mathrm{~B}-\mathrm{C} 24 \mathrm{~B}-\mathrm{C} 23 \mathrm{~B} & 58(2) & \mathrm{C} 2-\mathrm{L}-\mathrm{O} 2 \mathrm{~B}-\mathrm{C} 52 \mathrm{~B} & 174(6) \\ \mathrm{C} 22 \mathrm{~B}-\mathrm{C} 23 \mathrm{~B}-\mathrm{C} 24 \mathrm{~B}-\mathrm{C} 19 \mathrm{~B} & -59(2) & \mathrm{C} 1-\mathrm{L}-\mathrm{O} 2 \mathrm{~B}-\mathrm{C} 52 \mathrm{~B} & 60(8) \\ \mathrm{C} 2-\mathrm{N} 4-\mathrm{C} 25-\mathrm{C} 30 & -95.9(5) & \mathrm{HO}-\mathrm{LI}-\mathrm{O} 2 \mathrm{~B}-\mathrm{C} 52 \mathrm{~B} & 117(6) \\ \mathrm{C} 2-\mathrm{N} 4-\mathrm{C} 25-\mathrm{C} 26 & 144.9(4) & \mathrm{C} 55 \mathrm{~B}-\mathrm{O} 2 \mathrm{~B}-\mathrm{C} 52 \mathrm{~B}-\mathrm{C} 53 \mathrm{~B} & -21(8) \\ \mathrm{N} 4-\mathrm{C} 25-\mathrm{C} 26-\mathrm{C} 27 & 63.1(6) & \mathrm{L}-\mathrm{O} 2 \mathrm{~B}-\mathrm{C} 52 \mathrm{~B}-\mathrm{C} 53 \mathrm{~B} & 143(6) \\ \mathrm{C} 30-\mathrm{C} 25-\mathrm{C} 26-\mathrm{C} 27 & -57.1(6) & \mathrm{O} 2 \mathrm{~B}-\mathrm{C} 52 \mathrm{~B}-\mathrm{C} 53 \mathrm{~B}-\mathrm{C} 54 \mathrm{~B} & 21(6) \\ \mathrm{C} 25-\mathrm{C} 26-\mathrm{C} 27-\mathrm{C} 28 & 57.3(6) & \mathrm{C} 52 \mathrm{~B}-\mathrm{C} 53 \mathrm{~B}-\mathrm{C} 54 \mathrm{~B}-\mathrm{C} 55 \mathrm{~B} & -14(6) \\ \mathrm{C} 26-\mathrm{C} 27-\mathrm{C} 28-\mathrm{C} 29 & -55.2(6) & \mathrm{C} 52 \mathrm{~B}-\mathrm{O} 2 \mathrm{~B}-\mathrm{C} 55 \mathrm{~B}-\mathrm{C} 54 \mathrm{~B} & 13(9) \\ \mathrm{C} 27-\mathrm{C} 28-\mathrm{C} 29-\mathrm{C} 30 & 54.6(6) & \mathrm{L}-\mathrm{O} 2 \mathrm{~B}-\mathrm{C} 55 \mathrm{~B}-\mathrm{C} 54 \mathrm{~B} & -151(6) \\ \mathrm{C} 28-\mathrm{C} 29-\mathrm{C} 30-\mathrm{C} 25 & -55.5(6) & \mathrm{C} 53 \mathrm{~B}-\mathrm{C} 54 \mathrm{~B}-\mathrm{C} 55 \mathrm{~B}-\mathrm{O} 2 \mathrm{~B} & 1(8)\end{array}$

